\title{
Kinematical superspaces
}

\section{José Figueroa-O'Farrill and Ross Grassie}

Maxwell Institute and School of Mathematics, The University of Edinburgh, James Clerk Maxwell Building, Peter Guthrie Tait Road, Edinburgh EH9 3FD, Scotland, U.K.

E-mail: j.m.figueroa@ed.ac.uk, s1131494@sms.ed.ac.uk

ABSTRACT: We classify $N=1 d=4$ kinematical and aristotelian Lie superalgebras with spatial isotropy, but not necessarily parity nor time-reversal invariance. Employing a quaternionic formalism which makes rotational covariance manifest and simplifies many of the calculations, we find a list of 43 isomorphism classes of Lie superalgebras, some with parameters, whose (nontrivial) central extensions are also determined. We then classify their corresponding simply-connected homogeneous (4|4)-dimensional superspaces, resulting in a list of 27 homogeneous superspaces, some with parameters, all of which are reductive. We determine the invariants of low rank and explore how these superspaces are related via geometric limits.

KEYwords: Space-Time Symmetries, Superspaces

ArXiv EPrint: 1908.11278 


\section{Contents}

1 Introduction 1

2 Basic definitions and the statement of the problem 5

2.1 Kinematical Lie algebras 5

2.2 Kinematical Lie superalgebras $\quad 6$

$\begin{array}{lll}2.3 \text { Lie superalgebra brackets } & 7\end{array}$

$\begin{array}{ll}2.4 & \text { Some preliminary results }\end{array}$

2.5 Automorphisms 11

3 The classifications of kinematical and aristotelian Lie superalgebras $\quad \mathbf{1 3}$

$\begin{array}{ll}3.1 & \text { Classification of kinematical Lie superalgebras } \\ \end{array}$

$\begin{array}{ll}3.2 & \text { Classification of aristotelian Lie superalgebras } 25\end{array}$

3.3 Unpacking the quaternionic notation 28

$\begin{array}{lll}3.4 & \text { Central extensions } & 30\end{array}$

3.5 Automorphisms of kinematical Lie superalgebras 31

4 Homogeneous superspaces $\quad 45$

4.1 Homogeneous supermanifolds $\quad 45$

$\begin{array}{lll}4.2 & \text { Admissible super Lie pairs } & 49\end{array}$

$\begin{array}{lll}4.3 & \text { Effective super Lie pairs } & 52\end{array}$

$\begin{array}{lll}4.4 & \text { Aristotelian homogeneous superspaces } & 56\end{array}$

$\begin{array}{lll}4.5 & \text { Summary } & 57\end{array}$

$\begin{array}{ll}4.6 & \text { Low-rank invariants } \\ \end{array}$

5 Limits between superspaces $\quad 60$

$\begin{array}{lll}5.1 \text { Contractions of the AdS superalgebra } & 61\end{array}$

$\begin{array}{lll}5.2 & \text { Remaining galilean superspaces } & 62\end{array}$

$\begin{array}{lll}5.3 & \text { Aristotelian limits } & 65\end{array}$

$\begin{array}{lll}5.4 \text { Summary } & 67\end{array}$

$\begin{array}{lll}6 & \text { Conclusions } & 68\end{array}$

$\begin{array}{ll}\text { A Lorentzian superspaces } & \mathbf{7 0}\end{array}$

$\begin{array}{ll}\text { A.1 Minkowski superspace } & 70\end{array}$

$\begin{array}{lll}\text { A.2 } & \text { Anti de Sitter superspace } & 71\end{array}$

$\begin{array}{lll}\text { A.3 Einstein static superspace } & 72\end{array}$ 


\section{Introduction}

Four-dimensional rigid supersymmetry first appeared in 1971 in a paper [1] of Golfand and Likhtman, which is to our knowledge the first appearance of what is now known as the $N=1$ $d=4$ Poincaré superalgebra. A few years later, Zumino [2] studied rigid supersymmetry in $\mathrm{AdS}_{4}$, based on the simple Lie superalgebra $\mathfrak{o s p}(1 \mid 4)$. For many years these two were the only known $N=1 d=4$ Lie superalgebras. They are both (10|4)-dimensional, and, in fact, the Poincaré superalgebra can be exhibited as a contraction of $\mathfrak{o s p}(1 \mid 4)$ à la Inönü-Wigner. If we wish to extend $(N=1 d=4)$ supersymmetry beyond Minkowski and anti de Sitter spacetimes, we are faced with a choice. One can study $N=1$ supersymmetry algebras associated to other four-dimensional lorentzian manifolds, as in the Lie algebraic approach of [3], which results in Lie superalgebras which are filtered deformations of subalgebras of the Poincaré superalgebra. These filtered deformations have dimension $(n \mid 4)$ for $n \leq 10$, and hence, in most cases, some of the spacetime symmetry is broken. A second approach, which is the one taken here, is to keep the dimension of the superalgebra fixed at $(10 \mid 4)$, but sacrificing the existence of a lorentzian metric.

In short, the present paper extends (in dimension four) the recent classification [4] of spatially-isotropic homogeneous spacetimes, whose geometric properties were further studied in [5], to a classification of (4|4)-dimensional simply-connected spatially-isotropic homogeneous superspaces. In particular, we classify the (10|4)-dimensional Lie superalgebras with spatial isotropy. (See later for a precise definition.)

It is a natural question to ask, as Bacry and Lévy-Leblond did half a century ago [6], what the possible kinematics are. This question translates into the geometric problem of classifying the spacetimes which admit a transitive action of a kinematical Lie group. To answer this question, one first needs to classify kinematical Lie groups and then study their possible homogeneous spaces. If we allow the ambiguity of classifying homogeneous spaces up to coverings (or, equivalently, classifying the simply-connected homogeneous spaces), this problem has a largely algebraic solution: namely, the classification of pairs $(\mathfrak{k}, \mathfrak{h})$, where $\mathfrak{k}$ is a kinematical Lie algebra and $\mathfrak{h}$ a suitable subalgebra. With every such pair $(\mathfrak{k}, \mathfrak{h})$ (subject to some mild conditions) there is associated a unique simply-connected homogeneous space $M=\mathcal{K} / \mathcal{H}$, where $\mathcal{K}$ is a simply-connected (and connected) kinematical Lie group with Lie algebra $\mathfrak{k}$ and $\mathcal{H}$ is the connected subgroup generated by $\mathfrak{h}$. On $M$, the generators of $\mathfrak{k}$ act as infinitesimal rotations, boosts and spatio-temporal translations, whereas the generators of $\mathfrak{h}$ act as infinitesimal rotations and boosts about a choice of "origin" determined by the subgroup $\mathcal{H}$ itself.

Let us restrict ourselves to the case of four spacetime dimensions. In their pioneering paper [6], Bacry and Lévy-Leblond presented a classification of kinematical Lie algebras subject to the assumptions of the existence of automorphisms interpretable as parity and time-reversal. These "by no means compelling" assumptions were removed in [7], resulting in the classification of kinematical Lie algebras (with spatial isotropy) up to isomorphism. Already in these papers, the observation was made that every such kinematical Lie algebra $\mathfrak{k}$ (of dimension 10) admits a six-dimensional subalgebra $\mathfrak{h}$ so that the pair $(\mathfrak{k}, \mathfrak{h}$ ), if geometrically realisable as a homogeneous space, is a four-dimensional spatially isotropic 


\begin{tabular}{|c|c|c|c|c|c|c|}
\hline Label & \multicolumn{5}{|c|}{ Nonzero Lie brackets in addition to $[\boldsymbol{J}, \boldsymbol{J}]=\boldsymbol{J},[\boldsymbol{J}, \boldsymbol{B}]=\boldsymbol{B},[\boldsymbol{J}, \boldsymbol{P}]=\boldsymbol{P}$} & Comments \\
\hline$M^{4}$ & {$[H, \boldsymbol{B}]=-\boldsymbol{P}$} & & {$[\boldsymbol{B}, \boldsymbol{B}]=-\boldsymbol{J}$} & {$[\boldsymbol{B}, \boldsymbol{P}]=H$} & & Minkowski \\
\hline $\mathrm{dS}_{4}$ & {$[H, \boldsymbol{B}]=-\boldsymbol{P}$} & {$[H, P]=-B$} & {$[\boldsymbol{B}, \boldsymbol{B}]=-\boldsymbol{J}$} & {$[\boldsymbol{B}, \boldsymbol{P}]=H$} & {$[P, P]=J$} & de Sitter \\
\hline $\mathrm{AdS}_{4}$ & {$[H, \boldsymbol{B}]=-\boldsymbol{P}$} & {$[H, P]=\boldsymbol{B}$} & {$[\boldsymbol{B}, \boldsymbol{B}]=-\boldsymbol{J}$} & {$[\boldsymbol{B}, \boldsymbol{P}]=H$} & {$[\boldsymbol{P}, \boldsymbol{P}]=-\boldsymbol{J}$} & anti de Sitter \\
\hline $\mathbb{E}^{4}$ & {$[H, \boldsymbol{B}]=\boldsymbol{P}$} & & {$[\boldsymbol{B}, \boldsymbol{B}]=\boldsymbol{J}$} & {$[\boldsymbol{B}, \boldsymbol{P}]=H$} & & euclidean \\
\hline$S^{4}$ & {$[H, \boldsymbol{B}]=\boldsymbol{P}$} & {$[H, P]=-\boldsymbol{B}$} & {$[\boldsymbol{B}, \boldsymbol{B}]=\boldsymbol{J}$} & {$[\boldsymbol{B}, \boldsymbol{P}]=H$} & {$[\boldsymbol{P}, \boldsymbol{P}]=\boldsymbol{J}$} & sphere \\
\hline$H^{4}$ & {$[H, \boldsymbol{B}]=\boldsymbol{P}$} & {$[H, P]=\boldsymbol{B}$} & {$[\boldsymbol{B}, \boldsymbol{B}]=\boldsymbol{J}$} & {$[\boldsymbol{B}, \boldsymbol{P}]=H$} & {$[\boldsymbol{P}, \boldsymbol{P}]=-\boldsymbol{J}$} & hyperbolic space \\
\hline G & {$[H, \boldsymbol{B}]=-\boldsymbol{P}$} & & & & & galilean spacetime \\
\hline dSG & {$[H, \boldsymbol{B}]=-\boldsymbol{P}$} & {$[H, P]=-\boldsymbol{B}$} & & & & galilean de Sitter $\left(\mathrm{dSG}=\mathrm{dSG}_{\gamma=-1}\right)$ \\
\hline $\mathrm{dSG}_{\gamma}$ & {$[H, \boldsymbol{B}]=-\boldsymbol{P}$} & {$[H, \boldsymbol{P}]=\gamma \boldsymbol{B}+(1+\gamma) \boldsymbol{P}$} & & & & torsional galilean de Sitter $(\gamma \in(-1,1])$ \\
\hline AdSG & {$[H, \boldsymbol{B}]=-\boldsymbol{P}$} & {$[H, \boldsymbol{P}]=\boldsymbol{B}$} & & & & galilean anti de Sitter $\left(\right.$ AdSG $=$ AdSG $\left._{\chi=0}\right)$ \\
\hline $\mathrm{AdSG}_{\chi}$ & {$[H, \boldsymbol{B}]=-\boldsymbol{P}$} & {$[H, \boldsymbol{P}]=\left(1+\chi^{2}\right) \boldsymbol{B}+2 \chi \boldsymbol{P}$} & & & & torsional galilean anti de Sitter $(\chi>0)$ \\
\hline C & & & & {$[\boldsymbol{B}, \boldsymbol{P}]=H$} & & carrollian spacetime \\
\hline dSC & & {$[H, P]=-\boldsymbol{B}$} & & {$[\boldsymbol{B}, \boldsymbol{P}]=H$} & {$[P, P]=J$} & carrollian de Sitter \\
\hline AdSC & & {$[H, P]=\boldsymbol{B}$} & & {$[\boldsymbol{B}, \boldsymbol{P}]=H$} & {$[\boldsymbol{P}, \boldsymbol{P}]=-\boldsymbol{J}$} & carrollian anti de Sitter \\
\hline LC & {$[H, \boldsymbol{B}]=\boldsymbol{B}$} & {$[H, P]=-\boldsymbol{P}$} & & {$[\boldsymbol{B}, \boldsymbol{P}]=H-\boldsymbol{J}$} & & carrollian light cone \\
\hline S & & & & & & aristotelian static \\
\hline TS & & {$[H, P]=\boldsymbol{P}$} & & & & torsional aristotelian static \\
\hline $\mathbb{R} \times S^{3}$ & & & & & {$[\boldsymbol{P}, \boldsymbol{P}]=\boldsymbol{J}$} & Einstein static universe \\
\hline $\mathbb{R} \times H^{3}$ & & & & & {$[\boldsymbol{P}, \boldsymbol{P}]=-\boldsymbol{J}$} & hyperbolic Einstein static universe \\
\hline
\end{tabular}

Table 1. Simply-connected spatially-isotropic homogeneous spacetimes.

homogeneous spacetime of a kinematical Lie group. The precise relation between pairs $(\mathfrak{k}, \mathfrak{h})$ and homogeneous spacetimes is a little subtle, and this problem was revisited in [4], arriving at the classification of simply-connected spatially-isotropic homogeneous spacetimes which is summarised in table 1 below. (The results in [4] are not restricted to four spacetime dimensions, but already in the four-dimensional case they refine and slightly correct the list in [7].) We choose a basis where $\mathfrak{k}$ is spanned ${ }^{1}$ by $\left\{J_{i}, B_{i}, P_{i}, H\right\}$ and $\mathfrak{h}$ is spanned by $\left\{J_{i}, B_{i}\right\}$, so that the pair $(\mathfrak{k}, \mathfrak{h})$ is uniquely determined by specifying the Lie brackets in this basis. We use a standard shorthand notation for the Lie brackets, where $[H, \boldsymbol{B}]=\boldsymbol{B}$ stands for $\left[H, B_{i}\right]=B_{i},[\boldsymbol{J}, \boldsymbol{B}]=\boldsymbol{B}$ stands for $\left[J_{i}, B_{j}\right]=\epsilon_{i j k} B_{k}$ and $[\boldsymbol{B}, \boldsymbol{P}]=H$ stands for $\left[B_{i}, P_{j}\right]=\delta_{i j} H$, et cetera. As already discussed in the original papers [6, 7], the set of isomorphism classes of kinematical Lie algebras is partially ordered by contractions, which manifest themselves geometrically as limits between the homogeneous spacetimes. Such limits are discussed at length in [4].

The homogeneous spacetimes in table 1 fall into different classes, depending on the invariant structures that they possess. From top to bottom, we have the lorentzian spacetimes, the riemannian spaces, the galilean spacetimes, the carrollian spacetimes and finally the aristotelian spacetimes. Aristotelian spacetimes are homogeneous spaces of aristotelian Lie groups, where the boosts are absent. Many aristotelian Lie algebras arise as quotients of kinematical Lie algebras by the ideal generated by the boosts, when the boosts do generate an ideal. However not all aristotelian Lie algebras arise in this way, which motivated their classification in [4].

The lorentzian spaces in the table $\left(\mathrm{M}^{4}, \mathrm{dS}_{4}\right.$ and $\left.\mathrm{AdS}_{4}\right)$ are maximally symmetric and homogeneous spaces of the Poincaré group, $\operatorname{Spin}(4,1) \cong \operatorname{Sp}(1,1)$ and $\operatorname{Spin}(3,2) \cong \operatorname{Sp}(4, \mathbb{R})$, respectively. The last two isomorphisms are the spin representations, which shows that whereas the irreducible spinor representation of $\operatorname{Spin}(3,2)$ is real and four-dimensional, that of $\operatorname{Spin}(4,1)$ is quaternionic and two-dimensional.

\footnotetext{
${ }^{1}$ The boosts generators $B_{i}$ are absent in the aristotelian spacetimes.
} 
This paper aims to answer the question of what are the possible "super-kinematics" (in four spacetime dimensions). We will give a full answer for the case of $N=1$ supersymmetry or, equivalently, for the case of four real supercharges. In other words, we classify the superspaces which superise the homogeneous spacetimes in table 1. More precisely, we classify (simply-connected, spatially-isotropic) (4|4)-dimensional homogeneous superspaces of kinematical Lie supergroups. As in the classical (i.e., non-supersymmetric) case, we will work at the algebraic level and will classify pairs $(\mathfrak{s}, \mathfrak{h})$, where $\mathfrak{s}$ is a kinematical Lie superalgebra and $\mathfrak{h}$ an admissible subalgebra, concepts which will be defined carefully in the paper.

In a way, the superspaces in this paper belong to the same family as the well-known Minkowski and AdS superspaces, which are recalled in appendix A. Two features shared by these two superspaces is that their corresponding Lie superalgebras $\mathfrak{s}=\mathfrak{s}_{\overline{0}} \oplus \mathfrak{s}_{\overline{1}}$ are such that $\mathfrak{s}_{0}$ is a kinematical Lie algebra (Poincaré and $\mathfrak{s o}(3,2)$, respectively) and the odd subspace $\mathfrak{s}_{\overline{1}}$ is a four-dimensional real representation of $\mathfrak{s}_{\overline{0}}$ whose restriction to the rotational subalgebra $\mathfrak{r} \subset \mathfrak{s}_{\overline{0}}$ is the four-dimensional real spinor representation of $\mathfrak{r} \cong \mathfrak{s p}(1)$; that is, it is the one-dimensional quaternionic representation of $\mathfrak{s p}(1)$ but thought of as a real vector space. We will say that $\mathfrak{s}$ is an $N=1$ supersymmetric extension of the kinematical Lie algebra $\mathfrak{s}_{\overline{0}}$ or a kinematical Lie superalgebra, for short. One of the main results in this paper is the classification of kinematical Lie superalgebras up to isomorphism.

We are certainly not the first authors to ask what are the possible "super-kinematics" and indeed there are papers [8-11] which give partial answers to that question. In [8] the authors depart from the list of kinematical Lie algebras in [6] and consider their $N=1$ supersymmetric extensions while still requiring the existence of automorphisms corresponding to parity and time-reversal. They do this by solving the Jacobi identities for the superalgebra, having fixed the action of the rotational generators ab initio. Their list consists of those Lie superalgebras which can be obtained by contraction from the anti de Sitter superalgebra $\mathfrak{o s p}(1 \mid 4)$. In [9], among other results unrelated to the present paper, the authors study some of the contractions of the anti de Sitter superalgebra osp $(1 \mid 4)$, paying particular attention to (para-)Poincaré, galilean and Newton-Hooke superalgebras. In [10], the authors classify the kinematical contractions of $\mathfrak{o s p}(1 \mid 4)$ (and also of the corresponding anti de Sitter Lie algebras of order 3) and arrive at supersymmetric extensions of the Poincaré, galilean, Carroll and Newton-Hooke Lie algebras. Finally, in [11] the authors classify the contractions of $\mathfrak{o s p}(1 \mid 4)$ and in addition contract the associated superspaces. Some of these contractions, particularly those which result in a galilean superalgebra have also been studied by other authors (see, e.g., [12-15]); although in some cases the resulting superalgebra is not an extension of the galilean algebra but its universal central extension, the Bargmann algebra.

In this paper, we give a fuller answer to the question, in that we do not require the existence of parity nor time-reversal automorphisms and hence we depart not from the kinematical Lie algebras in [6], but from those in $[7,16]$. In particular, our list of Lie superalgebras includes, but substantially extends, the Lie superalgebras which can be constructed as contractions of $\mathfrak{o s p}(1 \mid 4)$. Our approach is as follows. We will first classify (up to isomorphism) the $N=1$ supersymmetric extensions of the kinematical Lie algebras 
(with three-dimensional space isotropy) listed, for convenience, in table 2. We work in full generality, but in the end restrict attention to those superalgebras where the bracket $\left[\mathfrak{s}_{\overline{1}}, \mathfrak{s}_{\overline{1}}\right]$ is nonzero. We solve this problem by employing a uniform quaternionic formalism for all kinematical Lie algebras and solving the Jacobi identities. The isomorphism classes of kinematical Lie superalgebras are listed in table 4, which is the first main result in this paper. We then classify the (effective, geometrically realisable) pairs $(\mathfrak{s}, \mathfrak{h}$ ) where $\mathfrak{s}$ is a kinematical Lie superalgebra and $\mathfrak{h} \subset \mathfrak{s}_{\overline{0}}$ an admissible subalgebra. As we will show, the pair $(\mathfrak{s}, \mathfrak{h})$ defines a homogeneous supermanifold which "superises" the homogeneous spacetime described by $\left(\mathfrak{s}_{\overline{0}}, \mathfrak{h}\right)$. The list of homogeneous superspaces is contained in table 14, which is the ultimate goal of this paper and contains our answer to the question of what are the possible (4|4)-dimensional "super-kinematics". Figure 1 illustrates the different limits which relate these superspaces.

Reader's guide. The paper is organised as follows. In section 2, we define the objects of interest and state the classification problems that we will solve in this paper. In section 2.2, we define kinematical Lie superalgebras and set up the quaternionic formalism we will employ throughout the paper. In section 2.3 , we set out the strategy we shall follow in classifying kinematical superalgebras. In section 2.4, we collect some useful preliminary results we will use often and in section 2.5 we discuss the nature of the automorphisms of kinematical superalgebras.

In section 3, we classify the kinematical and aristotelian Lie superalgebras, arriving at tables 4 and 6 . In doing so, we had to determine the automorphisms of the kinematical Lie algebras, which are summarised in table 3. Once having classified the kinematical and aristotelian Lie superalgebras, we determine their (nontrivial) central extensions in section 3.4. For later use, we need to determine the automorphisms of the Lie superalgebras (which fix the rotational subalgebra) and this is done in section 3.5.

In section 4 , we classify the pairs $(\mathfrak{s}, \mathfrak{h})$ and hence the simply-connected homogeneous superspaces, which are listed in table 14. In that table we list, in particular, the underlying homogeneous kinematical or aristotelian spacetime for each of our superspaces. In section 4.6, we determine the invariant tensors of low rank in each of the superspaces in table 14. In section 5, we explore how the superspaces in table 14 are related via geometric limits, arriving at the picture in figure 1 , which is to be contrasted with the similar picture (see figure 2) for the homogeneous spacetimes. Finally, in section 6, we offer some conclusions and point to possible extensions of this work.

This paper contains the details of two classifications: kinematical Lie superalgebras and their associated superspaces. As such it is somewhat lengthy and somewhat technical. Readers who are pressed for time might benefit from some hints about navigating the paper towards the main results. In order to arrive at these results we reformulated the problem in terms of quaternions and this formalism is described in section 2.2. The Lie algebraic classifications are the subject of section 3, but the main results are table 4 for the kinematical superalgebras and table 6 for the aristotelian superalgebras. The (nontrivial) central extensions are tabulated in table 7 . Section 4 contains the classification of the superspaces, starting with section 4.1, which explains the infinitesimal description of the superspaces 
in terms of super Lie pairs $(\mathfrak{s}, \mathfrak{h})$, and ending with table 14 , which lists the superspaces together with the underlying spacetime and a description of the corresponding Lie superalgebra. Section 5 discusses how these superspaces relate to each other via geometric limits, leading to figure 1 . The figures and the tables are hyperlinked for ease of navigation.

\section{Basic definitions and the statement of the problem}

In this section, we set up the classification problems of kinematical Lie superalgebras and homogeneous kinematical superspaces and introduce the quaternionic formalism we shall employ in the rest of the paper.

\subsection{Kinematical Lie algebras}

Let $\mathfrak{k}$ be a kinematical Lie algebra (with three-dimensional spatial isotropy). It is a real 10-dimensional Lie algebra with a subalgebra $\mathfrak{r} \cong \mathfrak{s o}(3)$ and such that under the adjoint action of $\mathfrak{r}, \mathfrak{k}$ decomposes as $\mathfrak{k}=\mathfrak{r} \oplus 2 V \oplus \mathbb{R}$, where $V$ is the three-dimensional irreducible vector representation of $\mathfrak{r}$ and $\mathbb{R}$ is the trivial one-dimensional scalar representation. A real basis for $\mathfrak{k}$ is given by $J_{i}, B_{i}, P_{i}$ and $H$, where $i=1,2,3$, where $J_{i}$ span $\mathfrak{r}, B_{i}$ and $P_{i}$ span the two copies of $V$ and $H$ is a scalar generator. The Lie brackets common to all kinematical Lie algebras are (using summation convention):

$$
\left[J_{i}, J_{j}\right]=\epsilon_{i j k} J_{k} \quad\left[J_{i}, B_{j}\right]=\epsilon_{i j k} B_{k} \quad\left[J_{i}, P_{j}\right]=\epsilon_{i j k} P_{k} \quad \text { and } \quad\left[J_{i}, H\right]=0 .
$$

Such kinematical Lie algebras were classified up to isomorphism by Bacry and Nuyts [7] (see also [16]) completing the earlier classification of Bacry and Lévy-Leblond [6] of kinematical Lie algebras admitting time-reversal and parity automorphisms. Table 2 summarises the classification by listing the Lie brackets, in addition to the ones in equation (2.1). We use the by now standard abbreviated notation, where the vector indices are not explicitly written down, so that, for instance,

$$
\begin{array}{lll}
{[H, \boldsymbol{B}]=-\boldsymbol{P}} & \text { stands for } & {\left[H, B_{i}\right]=-P_{i}} \\
{[\boldsymbol{B}, \boldsymbol{P}]=H} & \text { stands for } & {\left[B_{i}, P_{j}\right]=\delta_{i j} H} \\
{[\boldsymbol{P}, \boldsymbol{P}]=\boldsymbol{J}} & \text { stands for } & {\left[P_{i}, P_{j}\right]=\epsilon_{i j k} J_{k},}
\end{array}
$$

et cetera. In this abbreviated notation, the brackets in equation (2.1) are written as

$$
[\boldsymbol{J}, \boldsymbol{J}]=\boldsymbol{J} \quad[\boldsymbol{J}, \boldsymbol{B}]=\boldsymbol{B} \quad[\boldsymbol{J}, \boldsymbol{P}]=\boldsymbol{P} \quad \text { and } \quad[\boldsymbol{J}, H]=0 .
$$

The one-parameter families $3_{\gamma}$ and $4_{\chi}$ of kinematical Lie algebras extend the lorentzian and euclidean Newton-Hooke Lie algebras, which correspond to $3_{\gamma=-1}$ and $4_{\chi=0}$, respectively.

It should be remarked that the correspondence between kinematical Lie algebras and their (simply-connected) homogeneous spacetimes is not bijective: there are kinematical Lie algebras with no associated homogeneous spacetimes and, conversely, there are kinematical Lie algebras with which there are associated more than one homogeneous spacetime. In describing the spacetimes in table 1, we have changed basis in the kinematical Lie algebra $\mathfrak{k}$ to ensure that the stabiliser subalgebra $\mathfrak{h}$ is always spanned by $J_{i}$ and $B_{i}$. This explains any perceived discrepancy between tables 1 and 2 . 


\begin{tabular}{|c|c|c|c|c|c|c|}
\hline $\mathrm{K} \#$ & \multicolumn{5}{|c|}{ Nonzero Lie brackets (besides $[\boldsymbol{J},-]$ brackets) } & Comment \\
\hline 1 & & & & & & static \\
\hline 2 & {$[H, \boldsymbol{B}]=-\boldsymbol{P}$} & & & & & galilean \\
\hline $3_{\gamma \in[-1,1]}$ & {$[H, \boldsymbol{B}]=\gamma \boldsymbol{B}$} & {$[H, \boldsymbol{P}]=\boldsymbol{P}$} & & & & \\
\hline $4_{\chi} \geq 0$ & {$[H, \boldsymbol{B}]=\chi \boldsymbol{B}+\boldsymbol{P}$} & {$[H, \boldsymbol{P}]=\chi \boldsymbol{P}$} & & & & \\
\hline 5 & {$[H, \boldsymbol{B}]=\boldsymbol{B}+\boldsymbol{P}$} & {$[H, \boldsymbol{P}]=\boldsymbol{P}$} & & & & \\
\hline 6 & & & {$[\boldsymbol{B}, \boldsymbol{P}]=H$} & & & Carroll \\
\hline 7 & {$[H, \boldsymbol{B}]=\boldsymbol{P}$} & & {$[\boldsymbol{B}, \boldsymbol{P}]=H$} & {$[\boldsymbol{B}, \boldsymbol{B}]=\boldsymbol{J}$} & & euclidean \\
\hline 8 & {$[H, \boldsymbol{B}]=-\boldsymbol{P}$} & & {$[\boldsymbol{B}, \boldsymbol{P}]=H$} & {$[\boldsymbol{B}, \boldsymbol{B}]=-\boldsymbol{J}$} & & Poincaré \\
\hline 9 & {$[H, B]=\boldsymbol{B}$} & {$[H, \boldsymbol{P}]=-\boldsymbol{P}$} & {$[\boldsymbol{B}, \boldsymbol{P}]=H$} & & & $\mathfrak{s o}(4,1)$ \\
\hline 10 & {$[H, \boldsymbol{B}]=\boldsymbol{P}$} & {$[H, \boldsymbol{P}]=-\boldsymbol{B}$} & {$[\boldsymbol{B}, \boldsymbol{P}]=H$} & {$[\boldsymbol{B}, \boldsymbol{B}]=\boldsymbol{J}$} & {$[\boldsymbol{P}, \boldsymbol{P}]=\boldsymbol{J}$} & $\mathfrak{s o}(5)$ \\
\hline 11 & {$[H, \boldsymbol{B}]=-\boldsymbol{P}$} & {$[H, \boldsymbol{P}]=\boldsymbol{B}$} & {$[\boldsymbol{B}, \boldsymbol{P}]=H$} & {$[\boldsymbol{B}, \boldsymbol{B}]=-\boldsymbol{J}$} & {$[\boldsymbol{P}, \boldsymbol{P}]=-\boldsymbol{J}$} & $\mathfrak{s o}(3,2)$ \\
\hline 12 & & & & {$[\boldsymbol{B}, \boldsymbol{B}]=\boldsymbol{B}$} & {$[\boldsymbol{P}, \boldsymbol{P}]=\boldsymbol{B}-\boldsymbol{J}$} & \\
\hline 13 & & & & {$[\boldsymbol{B}, \boldsymbol{B}]=\boldsymbol{B}$} & {$[\boldsymbol{P}, \boldsymbol{P}]=\boldsymbol{J}-\boldsymbol{B}$} & \\
\hline 14 & & & & {$[\boldsymbol{B}, \boldsymbol{B}]=\boldsymbol{B}$} & & \\
\hline 15 & & & & {$[\boldsymbol{B}, \boldsymbol{B}]=\boldsymbol{P}$} & & \\
\hline 16 & & {$[H, \boldsymbol{P}]=\boldsymbol{P}$} & & {$[\boldsymbol{B}, \boldsymbol{B}]=\boldsymbol{B}$} & & \\
\hline 17 & {$[H, \boldsymbol{B}]=-\boldsymbol{P}$} & & & {$[\boldsymbol{B}, \boldsymbol{B}]=\boldsymbol{P}$} & & \\
\hline 18 & {$[H, \boldsymbol{B}]=\boldsymbol{B}$} & {$[H, \boldsymbol{P}]=2 \boldsymbol{P}$} & & {$[\boldsymbol{B}, \boldsymbol{B}]=\boldsymbol{P}$} & & \\
\hline
\end{tabular}

Table 2. Kinematical Lie algebras.

\subsection{Kinematical Lie superalgebras}

We start by defining the objects of interest.

Definition 1. An $(N=1)$ kinematical Lie superalgebra (with three-dimensional space isotropy) is a real Lie superalgebra $\mathfrak{s}=\mathfrak{s}_{\overline{0}} \oplus \mathfrak{s}_{\overline{1}}$, where $\mathfrak{s}_{\overline{0}}=\mathfrak{k}$ is a kinematical Lie algebra (with three-dimensional space isotropy) and $\mathfrak{s}_{\overline{1}}=S$, where $S$ is a representation of $\mathfrak{k}$ which extends the four-dimensional real spinor representation of the rotational subalgebra $\mathfrak{r}$.

Under the isomorphism $\mathfrak{r} \cong \mathfrak{s p}(1)$, we may take $S$ to be a copy of the quaternions and the action of $\mathfrak{r}$ on $S$ is essentially given by left quaternion multiplication. Let us be more

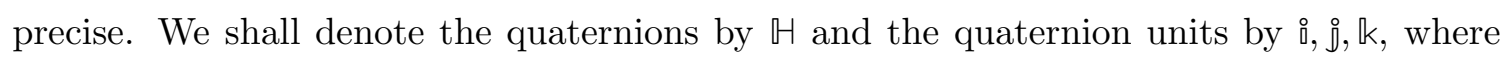
$\AA^{2}=-1, \AA^{2}=-1$ and $\stackrel{0}{j}=\mathbb{k}=-\stackrel{0}{0}$.

Let us define the following injective real linear maps (again using the summation convention):

$$
\begin{aligned}
& \mathrm{J}: \operatorname{Im} \mathbb{H} \rightarrow \mathfrak{s} \quad \text { given by } \quad \mathrm{J}(\omega)=\omega_{i} J_{i} \quad \text { for } \quad \omega=\omega_{1} \stackrel{0}{ }+\omega_{2} \AA+\omega_{3} \mathbb{k} \in \operatorname{Im} \mathbb{H} \\
& \mathrm{B}: \operatorname{Im} \mathbb{H} \rightarrow \mathfrak{s} \quad \text { given by } \quad \mathrm{B}(\beta)=\beta_{i} B_{i} \quad \text { for } \quad \beta=\beta_{1} \AA+\beta_{2} \AA+\beta_{3} \mathbb{k} \in \operatorname{Im} \mathbb{H} \\
& \mathrm{P}: \operatorname{Im} H \rightarrow \mathfrak{s} \quad \text { given by } \quad \mathrm{P}(\pi)=\pi_{i} P_{i} \quad \text { for } \quad \pi=\pi_{1} \stackrel{\imath}{ }+\pi_{2} \AA+\pi_{3} \mathbb{k} \in \operatorname{Im} \mathbb{H} \\
& \mathrm{Q}: \mathbb{H} \rightarrow \mathfrak{s} \quad \text { given by } \quad \mathrm{Q}(s)=s_{a} Q_{a} \quad \text { for } \quad s=s_{1} \AA+s_{2} \AA+s_{3} \mathbb{k}+s_{4} \in \mathbb{H} \text {, }
\end{aligned}
$$


where $\left(Q_{1}, Q_{2}, Q_{3}, Q_{4}\right)$ is a real basis for $\mathfrak{s}_{\overline{1}}$. The (nonzero) Lie brackets common to all kinematical Lie superalgebras are then given in terms of quaternion multiplication as follows:

$$
\begin{aligned}
& {\left[\mathrm{J}(\omega), \mathrm{J}\left(\omega^{\prime}\right)\right]=\frac{1}{2} \mathrm{~J}\left(\left[\omega, \omega^{\prime}\right]\right)} \\
& {[\mathrm{J}(\omega), \mathrm{B}(\beta)]=\frac{1}{2} \mathrm{~B}([\omega, \beta])} \\
& {[\mathrm{J}(\omega), \mathrm{P}(\pi)]=\frac{1}{2} \mathrm{P}([\omega, \pi])} \\
& {[\mathrm{J}(\omega), \mathrm{Q}(s)]=\frac{1}{2} \mathrm{Q}(\omega s)}
\end{aligned}
$$

where $\omega, \omega^{\prime}, \beta, \pi \in \operatorname{Im} H$ and $s \in \mathbb{H}$ and where $\omega s$ is the quaternion product and $[\omega, \beta]:=$ $\omega \beta-\beta \omega$, et cetera, are quaternion commutators. One can easily check that the Jacobi identities involving at least two vectors in $\mathfrak{r}$ are satisfied by virtue of the associativity of quaternion multiplication. For each kinematical Lie algebra $\mathfrak{k}$, the additional Lie brackets can also be written quaternionically. For example,

$$
\begin{aligned}
& {[H, \boldsymbol{B}]=-\boldsymbol{P} \quad \text { becomes } \quad[H, \mathrm{~B}(\beta)]=-\mathrm{P}(\beta)} \\
& {[\boldsymbol{B}, \boldsymbol{P}]=H \quad \text { becomes } \quad[\mathrm{B}(\beta), \mathrm{P}(\pi)]=\operatorname{Re}(\bar{\beta} \pi) H=-\operatorname{Re}(\beta \pi) H} \\
& {[\boldsymbol{P}, \boldsymbol{P}]=\boldsymbol{J} \quad \text { becomes } \quad\left[\mathrm{P}(\pi), \mathrm{P}\left(\pi^{\prime}\right)\right]=\frac{1}{2} \mathrm{~J}\left(\left[\pi, \pi^{\prime}\right]\right),}
\end{aligned}
$$

et cetera.

\subsection{Lie superalgebra brackets}

Let $\mathfrak{s}$ be a kinematical Lie superalgebra where $\mathfrak{s}_{\overline{0}}=\mathfrak{k}$ is a kinematical Lie algebra from table 2. To determine $\mathfrak{s}$ we need to specify the additional Lie brackets: $[H, \boldsymbol{Q}],[\boldsymbol{B}, \boldsymbol{Q}]$, $[\boldsymbol{P}, \boldsymbol{Q}]$ and $[\boldsymbol{Q}, \boldsymbol{Q}]$, subject to the Jacobi identity. There are four components to the Jacobi identity in a Lie superalgebra $\mathfrak{s}=\mathfrak{s}_{\overline{0}} \oplus \mathfrak{s}_{\overline{1}}$ :

1. The $\left(\mathfrak{s}_{\overline{0}}, \mathfrak{s}_{\overline{0}}, \mathfrak{s}_{\overline{0}}\right)$ Jacobi identity simply says that $\mathfrak{s}_{\overline{0}}$ is a Lie algebra, which in our case is one of the kinematical Lie algebras $\mathfrak{k}$ in table 2.

2. The $\left(\mathfrak{s}_{\overline{0}}, \mathfrak{s}_{\overline{0}}, \mathfrak{s}_{\overline{1}}\right)$ Jacobi identity says that $\mathfrak{s}_{\overline{1}}$ is a representation of $\mathfrak{s}_{\overline{0}}$ and, by restriction, also a representation of any Lie subalgebra of $\mathfrak{s}_{0}$ : for example, $\mathfrak{r}$ in our case.

3. The $\left(\mathfrak{s}_{\overline{0}}, \mathfrak{s}_{\overline{1}}, \mathfrak{s}_{\overline{1}}\right)$ Jacobi identity says that the component of the Lie bracket $\bigodot^{2} \mathfrak{s}_{\overline{1}} \rightarrow \mathfrak{s}_{\overline{0}}$ is $\mathfrak{s}_{0}$-equivariant. In particular, in our case, it is $\mathfrak{r}$-equivariant.

4. The $\left(\mathfrak{s}_{\overline{1}}, \mathfrak{s}_{\overline{1}}, \mathfrak{s}_{\overline{1}}\right)$ component does not seem to have any representation-theoretic reformulation and needs to be checked explicitly.

Our strategy will be the following. We shall first determine the space of $\mathfrak{r}$-equivariant brackets $[H, \boldsymbol{Q}],[\boldsymbol{B}, \boldsymbol{Q}],[\boldsymbol{P}, \boldsymbol{Q}]$ and $[\boldsymbol{Q}, \boldsymbol{Q}]$, which will turn out to be a 22-dimensional real vector space $\mathscr{V}$. For each kinematical Lie algebra $\mathfrak{k}=\mathfrak{s}_{\overline{0}}$ in table 2 , we then determine the algebraic variety $\mathscr{J} \subset \mathscr{V}$ cut out by the Jacobi identity. We are eventually interested in supersymmetry algebras and hence we will restrict attention to Lie superalgebras $\mathfrak{s}$ for which $[\boldsymbol{Q}, \boldsymbol{Q}] \neq 0$, which define a sub-variety $\mathscr{S} \subset \mathscr{J}$. The isomorphism classes of kinematical Lie superalgebras (with $[\boldsymbol{Q}, \boldsymbol{Q}] \neq 0$ ) are in one-to-one correspondence with the orbits of 
$\mathscr{S}$ under the subgroup $G \subset \mathrm{GL}\left(\mathfrak{s}_{\overline{0}}\right) \times \mathrm{GL}\left(\mathfrak{s}_{\overline{1}}\right)$ which acts by automorphisms of $\mathfrak{k}=\mathfrak{s}_{\overline{0}}$, since we have fixed $\mathfrak{k}$ from the start. The group $G$ contains not just the automorphisms of the kinematical Lie algebra $\mathfrak{k}$ which act trivially on $\mathfrak{r}$, but also automorphisms which are induced by automorphisms of the quaternion algebra. We shall return to an explicit description of such automorphisms below.

Let us start by determining the $\mathfrak{r}$-equivariant brackets: $[H, \boldsymbol{Q}],[\boldsymbol{B}, \boldsymbol{Q}],[\boldsymbol{P}, \boldsymbol{Q}]$ and $[\boldsymbol{Q}, \boldsymbol{Q}]$. The bracket $[H, \boldsymbol{Q}]$ is an $\mathfrak{r}$-equivariant endomorphism of the spinor module $\boldsymbol{Q}$. If we identify $\mathfrak{r}$ with the imaginary quaternions and $\boldsymbol{Q}$ with the quaternions, the action of $\mathfrak{r}$ on $\boldsymbol{Q}$ is via left quaternion multiplication. The endomorphisms of the representation $S$ which commute with the action of $\mathfrak{r}$ consist of left multiplication by reals and right multiplication by quaternions, but for real numbers, left and right multiplications agree, since the reals are central in the quaternion algebra. Hence the most general $\mathfrak{r}$-equivariant $[H, \boldsymbol{Q}]$ bracket takes the form

$$
[H, \mathrm{Q}(s)]=\mathrm{Q}(s \mathfrak{h}) \quad \text { for some } \quad \mathbb{h}=h_{1} \AA+h_{2} \AA+h_{3} \mathbb{k}+h_{4} \in \mathbb{H} .
$$

The brackets $[\boldsymbol{B}, \boldsymbol{Q}]$ and $[\boldsymbol{P}, \boldsymbol{Q}]$ are $\mathfrak{r}$-equivariant homomorphisms $V \otimes S \rightarrow S$, where $V$ and $S$ are the vector and spinor modules of $\mathfrak{s o}(3)$. There is an $\mathfrak{r}$-equivariant map $V \otimes S \rightarrow S$ given by the "Clifford action", which in this language is left multiplication by Im $\leftrightarrow$ on $\leftrightarrow$. Its kernel is the 8-dimensional real representation $W$ of $\mathfrak{r}$ with spin $\frac{3}{2}$. Therefore, the space of $\mathfrak{r}$-equivariant homomorphisms $V \otimes S \rightarrow S$ is isomorphic to the space of $\mathfrak{r}$-equivariant endomorphisms of $S$, which, as we saw before, is a copy of the quaternions. In summary, the $[\boldsymbol{B}, \boldsymbol{Q}]$ and $[\boldsymbol{P}, \boldsymbol{Q}]$ brackets take the form

$$
\begin{array}{lll}
{[\mathrm{B}(\beta), \mathrm{Q}(s)]=\mathrm{Q}(\beta s \mathbb{b})} & \text { for some } & \mathbb{b}=b_{1} \AA+b_{2} \AA+b_{3} \mathbb{k}+b_{4} \in \mathbb{H} \\
{[\mathrm{P}(\pi), \mathrm{Q}(s)]=\mathrm{Q}(\pi s \mathbb{p})} & \text { for some } & \mathbb{P}=p_{1} \AA+p_{2} \AA+p_{3} \mathbb{k}+p_{4} \in \mathbb{H},
\end{array}
$$

for all $\beta, \pi \in \operatorname{Im} \mathbb{H}$ and $s \in \mathbb{H}$.

Finally, we look at the $[\boldsymbol{Q}, \boldsymbol{Q}]$ bracket, which is an $\mathfrak{r}$-equivariant linear map $\bigodot^{2} S \rightarrow$ $\mathfrak{k}=\mathbb{R} \oplus 3 V$. The symmetric square $\bigodot^{2} S$ is a 10-dimensional representation of $\mathfrak{r}$ which decomposes as $\mathbb{R} \oplus 3 \mathrm{~V}$. Indeed, on $S$ we have an $\mathfrak{r}$-invariant inner product given by

$$
\left\langle s_{1}, s_{2}\right\rangle=\operatorname{Re}\left(\bar{s}_{1} s_{2}\right) \quad \text { where } \quad s_{1}, s_{2} \in \mathbb{H} .
$$

It is clearly invariant under left multiplication by unit quaternions: $\left\langle u s_{1}, u s_{2}\right\rangle=\left\langle s_{1}, s_{2}\right\rangle$ for all $u \in \operatorname{Sp}(1)$. We can use this inner product to identify $\bigodot^{2} S$ with the symmetric endomorphisms of $S$ : linear maps $\lambda: S \rightarrow S$ such that $\left\langle\lambda\left(s_{1}\right), s_{2}\right\rangle=\left\langle s_{1}, \lambda\left(s_{2}\right)\right\rangle$. Letting $L_{\mathbb{q}}$ and $R_{\mathbb{q}}$ denote left and right quaternion multiplication by $\mathbb{q} \in \mathbb{H}$, the space of symmetric

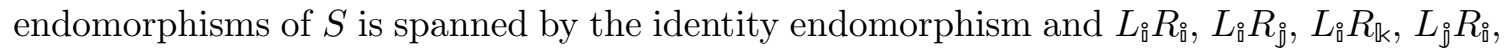
$L_{\S} R_{\S}, L_{\S} R_{\mathbb{k}}, L_{\mathbb{k}} R_{\mathfrak{\jmath}}, L_{\mathbb{k}} R_{\S}$ and $L_{\mathbb{k}} R_{\mathbb{k}}$. The nine non-identity symmetric endomorphisms transform under $\mathfrak{r}$ according to three copies of $V$. Since $\mathfrak{r}$ acts on $S$ via left multiplication, it commutes with the $R_{\mathscr{q}}$ and hence the three copies of $V$ are

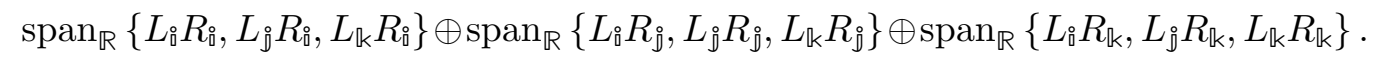


The space of $\mathfrak{r}$-equivariant linear maps $\bigodot^{2} S \rightarrow 3 V \oplus \mathbb{R}$ is thus isomorphic to the space of $\mathfrak{r}$-equivariant endomorphisms of $\mathbb{R} \oplus 3 V=\mathbb{R} \oplus\left(\mathbb{R}^{3} \otimes V\right)$, which is given by

$$
\operatorname{End}_{\mathfrak{r}}\left(\mathbb{R} \oplus\left(\mathbb{R}^{3} \otimes V\right)\right) \cong \operatorname{End}(\mathbb{R}) \oplus\left(\operatorname{End}\left(\mathbb{R}^{3}\right) \otimes \mathbb{1}_{V}\right)
$$

In summary, the $\mathfrak{r}$-equivariant $[\boldsymbol{Q}, \boldsymbol{Q}]$ bracket is given by polarisation from the following

$$
[\mathrm{Q}(s), \mathrm{Q}(s)]=c_{0}|s|^{2} H+\operatorname{Re}\left(\bar{s} \rrbracket s \mathbb{C}_{1}\right)+\operatorname{Re}\left(\bar{s} \mathbb{B} s \mathbb{C}_{2}\right)+\operatorname{Re}\left(\bar{s} \mathbb{P} s \mathbb{C}_{3}\right),
$$

where $c_{0} \in \mathbb{R}, \mathbb{C}_{1}, \mathbb{C}_{2}, \mathbb{C}_{3} \in \operatorname{Im} \mathbb{H}$ and where we have introduced the shorthands

$$
\mathbb{J}=J_{1} \AA+J_{2} \AA+J_{3} \mathbb{k}, \quad \mathbb{B}=B_{1} \AA+B_{2} \AA+B_{3} \mathbb{k}, \quad \text { and } \quad \mathbb{P}=P_{1} \AA+P_{2} \AA+P_{3} \mathbb{k} .
$$

Notice that if $\omega \in \operatorname{Im} H$, then $\mathrm{J}(\omega)=\operatorname{Re}(\bar{\omega} \mathbb{d})$, and similarly $\mathrm{B}(\beta)=\operatorname{Re}(\bar{\beta} \mathbb{B})$ and $\mathrm{P}(\pi)=$ $\operatorname{Re}(\bar{\pi} \mathbb{P})$, for $\beta, \pi \in \operatorname{Im} H$, so that we can rewrite the $[\boldsymbol{Q}, \boldsymbol{Q}]$ bracket as

$$
[\mathrm{Q}(s), \mathrm{Q}(s)]=c_{0}|s|^{2} H-\mathrm{J}\left(s \mathbb{C}_{1} \bar{s}\right)-\mathrm{B}\left(s \mathbb{C}_{2} \bar{s}\right)-\mathrm{P}\left(s \mathbb{C}_{3} \bar{s}\right),
$$

which polarises to give

$$
\left[\mathrm{Q}(s), \mathrm{Q}\left(s^{\prime}\right)\right]=c_{0} \operatorname{Re}\left(\bar{s} s^{\prime}\right) H-\frac{1}{2} \mathrm{~J}\left(s^{\prime} \mathbb{C}_{1} \bar{s}+s \mathbb{C}_{1} \bar{s}^{\prime}\right)-\frac{1}{2} \mathrm{~B}\left(s^{\prime} \mathbb{C}_{2} \bar{s}+s \mathbb{C}_{2} \bar{s}^{\prime}\right)-\frac{1}{2} \mathrm{P}\left(s^{\prime} \mathbb{C}_{3} \bar{s}+s \mathbb{C}_{3} \bar{s}^{\prime}\right) .
$$

In summary, we have that the $\mathfrak{r}$-equivariant brackets by which we extend the kinematical Lie algebra $\mathfrak{k}$ live in a 22 -dimensional real vector space of parameters $\mathbb{h}, \mathbb{b}, \mathbb{p} \in \mathbb{H}$, $\mathbb{C}_{1}, \mathbb{C}_{2}, \mathbb{C}_{3} \in \operatorname{Im} \mathbb{H}$ and $c_{0} \in \mathbb{R}$.

\subsection{Some preliminary results}

As mentioned above, one of the components of the Jacobi identity for the Lie superalgebra $\mathfrak{s}$ says that $\mathfrak{s}_{\overline{1}}$ is an $\mathfrak{s}_{\overline{0}}$-module, where $\mathfrak{s}_{\overline{0}}=\mathfrak{k}$ is the underlying kinematical Lie algebra. The Jacobi identity

$$
[X,[Y, \mathrm{Q}(s)]]-[Y,[X, \mathrm{Q}(s)]]=[[X, Y], \mathrm{Q}(s)] \quad \text { for all } X, Y \in \mathfrak{k}
$$

gives relations between the parameters $\mathbb{h}, \mathbb{b}, \mathbb{p} \in \mathbb{H}$ appearing in the Lie brackets.

Lemma 1. The following relations between $\mathbb{h}, \mathbb{b}, \mathbb{p} \in \mathbb{H}$ are implied by the corresponding $\mathfrak{k}$-brackets:

$$
\begin{aligned}
{[H, \boldsymbol{B}]=\lambda \boldsymbol{B}+\mu \boldsymbol{P} } & \Longrightarrow[\mathbb{b}, \mathfrak{h}]=\lambda \mathfrak{b}+\mu \mathfrak{p} \\
{[H, \boldsymbol{P}]=\lambda \boldsymbol{B}+\mu \boldsymbol{P} } & \Longrightarrow[\mathfrak{p}, \mathfrak{h}]=\lambda \mathfrak{b}+\mu \mathfrak{p} \\
{[\boldsymbol{B}, \boldsymbol{B}]=\lambda \boldsymbol{B}+\mu \boldsymbol{P}+\nu \boldsymbol{J} } & \Longrightarrow \mathbb{b}^{2}=\frac{1}{2} \lambda \mathfrak{b}+\frac{1}{2} \mu \mathfrak{p}+\frac{1}{4} \nu \\
{[\boldsymbol{P}, \boldsymbol{P}]=\lambda \boldsymbol{B}+\mu \boldsymbol{P}+\nu \boldsymbol{J} } & \Longrightarrow \mathbb{p}^{2}=\frac{1}{2} \lambda \mathfrak{b}+\frac{1}{2} \mu \mathfrak{p}+\frac{1}{4} \nu \\
{[\boldsymbol{B}, \boldsymbol{P}]=\lambda H } & \Longrightarrow \mathbb{b} \mathfrak{p}+\mathfrak{p} \mathfrak{b}=0 \quad \text { and } \quad[\mathbb{b}, \mathfrak{p}]=\lambda \mathfrak{h} .
\end{aligned}
$$

Proof. The $[H, \boldsymbol{B}, \boldsymbol{Q}]$ Jacobi identity says for all $\beta \in \operatorname{Im} \mathbb{H}$ and $s \in \mathbb{H}$,

$$
[[H, \mathrm{~B}(\beta)], \mathrm{Q}(s)]=[H,[\mathrm{~B}(\beta), \mathrm{Q}(s)]]-[\mathrm{B}(\beta),[H, \mathrm{Q}(s)]],
$$


which becomes

$$
\lambda \mathrm{Q}(\beta s \mathfrak{b})+\mu \mathrm{Q}(\beta s \mathrm{p})=\mathrm{Q}(\beta s \mathfrak{b} \mathfrak{h})-\mathrm{Q}(\beta s \mathfrak{h}) .
$$

Since $Q$ is real linear and injective, it follows that

$$
\lambda \beta s \mathfrak{b}+\mu \beta s p=\beta s[\mathfrak{b}, \mathfrak{h}],
$$

which, since it must hold for all $\beta \in \operatorname{Im} \nVdash$ and $s \in \mathbb{H}$, becomes

$$
[\mathfrak{b}, \mathfrak{h}]=\lambda \mathfrak{b}+\mu \mathfrak{p},
$$

as desired. Similarly, the $[H, \boldsymbol{P}, \boldsymbol{Q}]$ Jacobi identity gives the second equation in the lemma. The third equation follows from the $[\boldsymbol{B}, \boldsymbol{B}, \boldsymbol{Q}]$ Jacobi identity, which says that for all

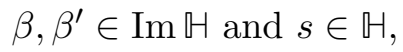

$$
\left[\left[\mathrm{B}(\beta), \mathrm{B}\left(\beta^{\prime}\right)\right], \mathrm{Q}(s)\right]=\left[\mathrm{B}(\beta),\left[\mathrm{B}\left(\beta^{\prime}\right), \mathrm{Q}(s)\right]\right]-\left[\mathrm{B}\left(\beta^{\prime}\right),[\mathrm{B}(\beta), \mathrm{Q}(s)]\right],
$$

which becomes

$$
\frac{1}{2} \lambda \mathrm{Q}\left(\left[\beta, \beta^{\prime}\right] s \mathbb{b}\right)+\frac{1}{2} \mu \mathrm{Q}\left(\left[\beta, \beta^{\prime}\right] s \mathbb{P}\right)+\frac{1}{4} \nu \mathrm{Q}\left(\left[\beta, \beta^{\prime}\right] s\right)=\mathrm{Q}\left(\beta \beta^{\prime} s \mathbb{b}^{2}\right)-\mathrm{Q}\left(\beta^{\prime} \beta s \mathbb{b}^{2}\right) .
$$

Again by linearity and injectivity of $\mathrm{Q}$, this is equivalent to

$$
\frac{1}{2} \lambda\left[\beta, \beta^{\prime}\right] s \mathfrak{b}+\frac{1}{2} \mu\left[\beta, \beta^{\prime}\right] s \mathfrak{p}+\frac{1}{4} \nu\left[\beta, \beta^{\prime}\right] s=\left[\beta, \beta^{\prime}\right] s \mathbb{b}^{2},
$$

which, being true for all $\beta, \beta^{\prime} \in \operatorname{Im} \nVdash$ and $s \in \mathbb{H}$, gives

$$
\frac{1}{2} \lambda \mathfrak{b}+\frac{1}{2} \mu \mathfrak{p}+\frac{1}{4} \nu=\mathfrak{b}^{2},
$$

as desired. The fourth identity in the lemma follows similarly from the $[\boldsymbol{P}, \boldsymbol{P}, \boldsymbol{Q}]$ Jacobi identity. Finally, we consider the $[\boldsymbol{B}, \boldsymbol{P}, \boldsymbol{Q}]$ Jacobi identity, which says that for all $\beta, \pi \in$ $\operatorname{Im} H$ and $s \in \mathbb{H}$,

$$
[[\mathrm{B}(\beta), \mathrm{P}(\pi)], \mathrm{Q}(s)]=[\mathrm{B}(\beta),[\mathrm{P}(\pi), \mathrm{Q}(s)]]-[\mathrm{P}(\pi),[\mathrm{B}(\beta), \mathrm{Q}(s)]],
$$

which expands to

$$
-\lambda \operatorname{Re}(\beta \pi) \mathrm{Q}(s \mathrm{~h})=\mathrm{Q}(\beta \pi s \mathrm{pb})-\mathrm{Q}(\pi \beta s \mathrm{bp})
$$

or, equivalently,

$$
-\lambda \operatorname{Re}(\beta \pi) s \mathrm{~h}=\beta \pi s \mathrm{plb}-\pi \beta s \mathrm{bp},
$$

for all $\beta, \pi \in \operatorname{Im} \leftrightarrow$ and $s \in \mathbb{H}$. For any two imaginary quaternions $\beta, \pi$, we have that

$$
\beta \pi=\frac{1}{2}[\beta, \pi]+\operatorname{Re}(\beta \pi),
$$

which allows us to rewrite equation (2.28) as

$$
\operatorname{Re}(\beta \pi) s(\lambda \mathfrak{h}-\mathbb{b} \mathfrak{p}+\mathfrak{p} \mathfrak{b})+\frac{1}{2}[\beta, \pi] s(\mathfrak{p l b}+\mathfrak{b} \mathfrak{p})=0 .
$$

Taking $\beta=\pi$ and $s=1$ we see that $\lambda \llbracket h=[\mathfrak{b}, \mathbb{p}]$ and taking $\beta$ and $\pi$ to be orthogonal and $s=1$, that $\mathfrak{p b}+\mathrm{b} p=0$, as desired. 
The components $[H, \boldsymbol{Q}, \boldsymbol{Q}],[\boldsymbol{B}, \boldsymbol{Q}, \boldsymbol{Q}]$ and $[\boldsymbol{P}, \boldsymbol{Q}, \boldsymbol{Q}]$ of the Jacobi identity are best studied on a case-by-case basis, but the $[\boldsymbol{Q}, \boldsymbol{Q}, \boldsymbol{Q}]$ component gives a universal condition.

Lemma 2. The $[\boldsymbol{Q}, \boldsymbol{Q}, \boldsymbol{Q}]$ component of the Jacobi identity implies

$$
c_{0} \mathfrak{h}=\frac{1}{2} \mathbb{C}_{1}+\mathbb{C}_{2} \mathfrak{b}+\mathbb{C}_{3} \mathbb{P} .
$$

Proof. The $[\boldsymbol{Q}, \boldsymbol{Q}, \boldsymbol{Q}]$ component of the Jacobi identity is totally symmetric and hence, by polarisation, it is uniquely determined by its value on the diagonal. In other words, it is equivalent to

$$
[[\mathrm{Q}(s), \mathrm{Q}(s)], \mathrm{Q}(s)] \stackrel{!}{=} 0 \quad \text { for all } s \in \mathbb{H} .
$$

Using equation (2.14), this becomes

$$
\left[c_{0}|s|^{2} H-\mathrm{J}\left(s \mathbb{C}_{1} \bar{s}\right)-\mathrm{B}\left(s \mathbb{C}_{2} \bar{s}\right)-\mathrm{P}\left(s \mathbb{C}_{3} \bar{s}\right), \mathrm{Q}(s)\right] \stackrel{!}{=} 0,
$$

which expands to

$$
c_{0}|s|^{2} \mathrm{Q}(s h)-\frac{1}{2} \mathrm{Q}\left(s \mathbb{C}_{1} \bar{s} s\right)-\mathrm{Q}\left(s \mathbb{C}_{2} \bar{s} s \mathbb{b}\right)-\mathrm{Q}\left(s \mathbb{C}_{3} \bar{s} s \mathrm{p}\right) \stackrel{!}{=} 0 .
$$

Since $Q$ is injective, this becomes

$$
|s|^{2} s\left(c_{0} \mathfrak{h}-\frac{1}{2} \mathbb{C}_{1}-\mathbb{C}_{2} \mathfrak{b}-\mathbb{C}_{3} \mathbb{P}\right) \stackrel{!}{=} 0 .
$$

This must hold for all $s \in \mathbb{H}$, so in particular for $s=1$, proving the lemma.

\subsection{Automorphisms}

As mentioned above, once we determine the sub-variety $\mathscr{S}$ cut out by the Jacobi identity, we need to quotient by the action of the subgroup $G \subset \mathrm{GL}\left(\mathfrak{s}_{\overline{0}}\right) \times \operatorname{GL}\left(\mathfrak{s}_{\overline{1}}\right)$ which acts by automorphisms of $\mathfrak{s}_{\overline{0}}=\mathfrak{k}$ in order to arrive at the isomorphism classes of Lie superalgebras. In this section, we describe the subgroup $G$ in more detail. There are two kinds of elements of $G$, those which act trivially on the rotational subalgebra $\mathfrak{r}$ and those which do not. The latter consist of inner automorphisms of $\mathfrak{k}$ which are generated infinitesimally by the adjoint action of $\boldsymbol{J}, \boldsymbol{B}$ and $\boldsymbol{P}$. The ones generated by $\boldsymbol{J}$ are particularly easy to describe in the quaternionic formulation, and we shall do so now in more detail.

Let $u \in \mathrm{Sp}(1)$ be a unit norm quaternion. Conjugation by $u$ defines a homomorphism $\operatorname{Ad}: \operatorname{Sp}(1) \rightarrow \operatorname{Aut}(\mathbb{H})$ whose kernel is the central subgroup of $\operatorname{Sp}(1)$ consisting of \pm 1 . It is a classical result that these are all the automorphisms of $\mathbb{H}$. Hence $\operatorname{Aut}(\mathbb{H}) \cong \operatorname{SO}(3)$, acting trivially on the real quaternions and rotating the imaginary quaternions. The action of Aut(H) on $\mathfrak{s}$ leaves $H$ invariant and acts on the remaining generators by pre-composing the linear maps $\mathrm{J}, \mathrm{B}, \mathrm{P}$ and $\mathrm{Q}$ with $\mathrm{Ad}_{u}$. More precisely, let $\widetilde{H}=H, \widetilde{\mathrm{J}}=\mathrm{J} \circ \mathrm{Ad}_{u}$, $\widetilde{\mathrm{B}}=\mathrm{B} \circ \mathrm{Ad}_{u}, \widetilde{\mathrm{P}}=\mathrm{P} \circ \mathrm{Ad}_{u}$ and $\widetilde{\mathrm{Q}}=\mathrm{Q} \circ \mathrm{Ad}_{u}$. Since the Lie brackets of $\mathfrak{k}$ are given in terms of quaternion multiplication, this transformation is an automorphism of $\mathfrak{k}$, and we have a group homomorphism Aut $(\mathbb{H}) \rightarrow \operatorname{Aut}(\mathfrak{k})$. The action on the remaining brackets (those 
involving $\boldsymbol{Q}$ ) is as follows. The Lie brackets of $\mathfrak{s}$ which involve $\boldsymbol{Q}$ are given by

$$
\begin{aligned}
{[H, \mathrm{Q}(s)] } & =\mathrm{Q}(s h) \\
{[\mathrm{J}(\omega), \mathrm{Q}(s)] } & =\frac{1}{2} \mathrm{Q}(\omega s) \\
{[\mathrm{B}(\beta), \mathrm{Q}(s)] } & =\mathrm{Q}(\beta s \mathbb{b}) \\
{[\mathrm{P}(\pi), \mathrm{Q}(s)] } & =\mathrm{Q}(\pi s \mathrm{P}) \\
{[\mathrm{Q}(s), \mathrm{Q}(s)] } & =c_{0}|s|^{2} H-\mathrm{J}\left(s \mathbb{C}_{1} \bar{s}\right)-\mathrm{B}\left(s \mathbb{C}_{2} \bar{s}\right)-\mathrm{P}\left(s \mathbb{C}_{3} \bar{s}\right),
\end{aligned}
$$

and hence under conjugation by $u \in \operatorname{Sp}(1)$,

$$
\begin{aligned}
{[\widetilde{H}, \widetilde{\mathrm{Q}}(s)] } & =\widetilde{\mathrm{Q}}(s \widetilde{\mathrm{h}}) \\
{[\widetilde{\mathrm{J}}(\omega), \widetilde{\mathrm{Q}}(s)] } & =\frac{1}{2} \widetilde{\mathrm{Q}}(\omega s) \\
{[\widetilde{\mathrm{B}}(\beta), \widetilde{\mathrm{Q}}(s)] } & =\widetilde{\mathrm{Q}}(\beta s \widetilde{\mathrm{b}}) \\
{[\widetilde{\mathrm{P}}(\pi), \widetilde{\mathrm{Q}}(s)] } & =\widetilde{\mathrm{Q}}(\pi s \widetilde{\mathrm{P}}) \\
{[\widetilde{\mathrm{Q}}(s), \widetilde{\mathrm{Q}}(s)] } & =c_{0}|s|^{2} \widetilde{H}-\widetilde{\mathrm{J}}\left(s \widetilde{\mathbb{C}}_{1} \bar{s}\right)-\widetilde{\mathrm{B}}\left(\widetilde{\mathbb{C}}_{2} \bar{s}\right)-\widetilde{\mathrm{P}}\left(s \widetilde{\mathbb{C}}_{3} \bar{s}\right),
\end{aligned}
$$

where $\widetilde{\mathfrak{h}}=\bar{u} \mathfrak{h} u, \widetilde{\mathrm{b}}=\bar{u} \mathfrak{b} u, \widetilde{\mathbb{p}}=\bar{u} \mathfrak{p} u$, and $\widetilde{\mathbb{C}}_{i}=\bar{u} \mathbb{c}_{i} u$ for $i=1,2,3$. In other words, the scalar parameters $c_{0}$, Re $\mathfrak{h}$, Re $b$ and $\operatorname{Re} p$ remain inert, but the imaginary quaternion parameters $\operatorname{Im} \mathfrak{h}, \operatorname{Im} \mathbb{b}, \operatorname{Im} \mathbb{p}, \mathbb{C}_{1,2,3}$ are simultaneously rotated. We will use these automorphisms very often in the sequel.

There are other automorphisms of $\mathfrak{k}$ which do transform $\mathfrak{r}$ : those are the inner automorphisms generated by $\boldsymbol{B}$ and $\boldsymbol{P}$. Their description depends on the precise form of $\mathfrak{k}$ but they will not play a rôle in our discussion.

In addition to these, $G$ also consists of automorphisms of $\mathfrak{k}$ which leave $\mathfrak{r}$ intact. If a linear map $\Phi: \mathfrak{s} \rightarrow \mathfrak{s}$ restricts to an automorphism of $\mathfrak{k}$, then it is in particular $\mathfrak{r}$ equivariant. The most general $\mathfrak{r}$-equivariant linear map $\Phi: \mathfrak{s} \rightarrow \mathfrak{s}$ sends $(\boldsymbol{J}, H, \boldsymbol{B}, \boldsymbol{P}, \boldsymbol{Q}) \mapsto$ $(\boldsymbol{J}, \widetilde{H}, \widetilde{\boldsymbol{B}}, \widetilde{\boldsymbol{P}}, \widetilde{\boldsymbol{Q}})$, where

$$
\begin{aligned}
\widetilde{H} & =\mu H \\
\widetilde{\mathrm{B}}(\beta) & =a \mathrm{~B}(\beta)+c \mathrm{P}(\beta)+e \mathrm{~J}(\beta) \\
\widetilde{\mathrm{B}}(\beta) & =b \mathrm{~B}(\beta)+d \mathrm{P}(\beta)+f \mathrm{~J}(\beta) \\
\widetilde{\mathrm{Q}}(s) & =\mathrm{Q}(s q)
\end{aligned}
$$

where $\mu \in \operatorname{GL}(1, \mathbb{R})=\mathbb{R}^{\times}, \mathbb{q} \in \mathrm{GL}(1, \mathbb{H})=\mathbb{H}^{\times}$and $\left(\begin{array}{lll}0 & a & b \\ 0 & c & d \\ 1 & e & f\end{array}\right) \in \mathrm{GL}(3, \mathbb{R})$. In $([4]$, subsection 3.1) we worked out the automorphisms (which fix $\mathfrak{r}$ ) of $\mathfrak{k}$ a kinematical Lie algebra isomorphic to one of $\mathrm{K} 1-\mathrm{K} 11$ in table 2 . The automorphisms of the remaining kinematical Lie algebras in the table are listed below (see table 3). In particular, we find that, although the precise form of the automorphisms depends on $\mathfrak{k}$, a common feature is that the coefficients $e, f$ are always zero, so we will set them to zero from now on without loss of generality. 
Assuming that the pair $\left(A=\left(\begin{array}{ll}a & b \\ c & d\end{array}\right), \mu\right) \in \mathrm{GL}(2, \mathbb{R}) \times \mathbb{R}^{\times}$is an automorphism of $\mathfrak{k}=\mathfrak{s}_{\overline{0}}$, the brackets involving $\boldsymbol{Q}$ change as follows:

$$
\begin{aligned}
{[\widetilde{H}, \widetilde{\mathrm{Q}}(s)] } & =\widetilde{\mathrm{Q}}(s \widetilde{\widehat{\complement}}) \\
{[\widetilde{\mathrm{B}}(\beta), \widetilde{\mathrm{Q}}(s)] } & =\widetilde{\mathrm{Q}}(\beta s \widetilde{\mathrm{B}}) \\
{[\widetilde{\mathrm{P}}(\pi), \widetilde{\mathrm{Q}}(s)] } & =\widetilde{\mathrm{Q}}(\pi s \widetilde{\mathbb{P}}) \\
{[\widetilde{\mathrm{Q}}(s), \widetilde{\mathrm{Q}}(s)] } & =\widetilde{c}_{0}|s|^{2} \widetilde{H}-\widetilde{\mathrm{J}}\left(s \widetilde{\mathbb{C}}_{1} \bar{s}\right)-\widetilde{\mathrm{B}}\left(s \widetilde{\mathbb{C}}_{2} \bar{s}\right)-\widetilde{\mathrm{P}}\left(s \widetilde{\mathbb{C}}_{3} \bar{s}\right),
\end{aligned}
$$

where $\widetilde{J}(\omega)=\mathbf{J}(\omega)$ and

$$
\begin{array}{rlrl}
\widetilde{\mathbb{h}} & =\mu \mathbb{q} \mathfrak{h} \mathbb{q}^{-1} & & \widetilde{\mathbb{C}}_{1}=\mathbb{a} \mathbb{C}_{1} \overline{\mathbb{q}} \\
\widetilde{\mathbb{b}} & =\mathbb{q}(a \mathfrak{b}+c \mathfrak{p}) \mathbb{q}^{-1} & & \widetilde{\mathbb{C}}_{2}=\frac{1}{a d-b c} \mathbb{q}\left(d \mathbb{\mathbb { C }}_{2}-b \mathbb{C}_{3}\right) \overline{\mathbb{q}} \\
\widetilde{\mathbb{P}} & =\mathbb{q}(b \mathfrak{b}+d \mathfrak{p}) \mathbb{q}^{-1} & & \widetilde{\mathbb{C}}_{3}=\frac{1}{a d-b c} \mathbb{q}\left(a \mathbb{C}_{3}-c \mathbb{C}_{2}\right) \overline{\mathbb{q}} . \\
\widetilde{c}_{0}=c_{0} \frac{|\mathbb{q}|^{2}}{\mu} & &
\end{array}
$$

In summary, the group $G$ by which we must quotient the sub-variety $\mathscr{S}$ cut out by the Jacobi identity (and $[\boldsymbol{Q}, \boldsymbol{Q}] \neq 0$ ) acts as follows on the generators:

$$
\begin{aligned}
\mathrm{J} & \mapsto \mathrm{J} \circ \mathrm{Ad}_{u} \\
\mathrm{~B} & \mapsto a \mathrm{~B} \circ \mathrm{Ad}_{u}+c \mathrm{P} \circ \mathrm{Ad}_{u} \\
\mathrm{P} & \mapsto b \mathrm{~B} \circ \mathrm{Ad}_{u}+d \mathrm{P} \circ \mathrm{Ad}_{u} \\
H & \mapsto \mu H \\
\mathrm{Q} & \mapsto \mathrm{Q} \circ \mathrm{Ad}_{u} \circ R_{\llbracket}
\end{aligned}
$$

where $\mu \in \mathbb{R}$ and $q \in \mathbb{H}$ are nonzero, $u \in \operatorname{Sp}(1)$ and $A:=\left(\begin{array}{ll}a & b \\ c & d\end{array}\right) \in \operatorname{GL}(2, \mathbb{R})$ with $(A, \mu)$ an automorphism of $\mathfrak{k}$.

Let $\operatorname{Aut}_{\mathfrak{r}}(\mathfrak{k})$ denote the subgroup of $\mathrm{GL}(2, \mathbb{R}) \times \mathbb{R}^{\times}$consisting of such $(A, \mu)$. These subgroups are listed in ([4], subsection 3.1) for the kinematical Lie algebras K1-K11 in table 2 . We will collect them in table 3 for convenience and in addition also record them for the remaining kinematical Lie algebras K12-K18 in table 2.

\section{The classifications of kinematical and aristotelian Lie superalgebras}

In this section, we classify the supersymmetric extensions of the kinematical Lie algebras in table 2. In addition, we will also classify aristotelian Lie superalgebras, as some of the homogeneous supermanifolds we will encounter later on will turn out to be superisations of the aristotelian homogeneous spacetimes classified in ([4], appendix A). 


\begin{tabular}{|c|c|}
\hline K\# & Typical $(A, \mu) \in \mathrm{GL}(2, \mathbb{R}) \times \mathbb{R}^{\times}$ \\
\hline 1 & $\left(\left(\begin{array}{ll}a & b \\
c & d\end{array}\right), \mu\right)$ \\
\hline 2 & $\left(\left(\begin{array}{ll}a & 0 \\
c & d\end{array}\right)\right.$, \\
\hline $3_{\gamma \in(-1,1)}$ & $\left(\left(\begin{array}{ll}a & 0 \\
0 & d\end{array}\right), 1\right.$ \\
\hline $3_{-1}$ & $\left(\left(\begin{array}{ll}a & 0 \\
0 & d\end{array}\right), 1\right),\left(\left(\begin{array}{ll}0 & b \\
c & 0\end{array}\right),-1\right)$ \\
\hline $3_{1}$ & $\left(\begin{array}{ll}a & b \\
c & d\end{array}\right)$ \\
\hline $4_{\chi>0}$ & $\left(\left(\begin{array}{cc}a & b \\
-b & a\end{array}\right)\right.$ \\
\hline $4_{0}$ & $\left(\left(\begin{array}{cc}a & b \\
-b & a\end{array}\right), 1\right),\left(\left(\begin{array}{cc}a & b \\
b & -a\end{array}\right),-1\right)$ \\
\hline 5 & $\left(\begin{array}{ll}a & 0 \\
c & a\end{array}\right), 1$ \\
\hline 6 & $\left(\left(\begin{array}{ll}a & b \\
c & d\end{array}\right), a d-b c\right)$ \\
\hline 7,8 & $\left.\left(\begin{array}{ll}1 & 0 \\
c & d\end{array}\right), d\right),\left(\left(\begin{array}{cc}-1 & 0 \\
c & d\end{array}\right),-d\right)$ \\
\hline 9 & $\left(\left(\begin{array}{cc}a & 0 \\
0 & a^{-1}\end{array}\right), 1\right),\left(\left(\begin{array}{cc}0 & b \\
b^{-1} & 0\end{array}\right),-1\right)$ \\
\hline 10,11 & $\left(\left(\begin{array}{cc}a & b \\
-b & a\end{array}\right), 1\right),\left(\left(\begin{array}{cc}a & b \\
b & -a\end{array}\right),-1\right), \quad a^{2}+b^{2}=1$ \\
\hline 12,13 & $\left(\left(\begin{array}{ll}1 & 0 \\
0 & 1\end{array}\right), \mu\right),\left(\left(\begin{array}{cc}1 & 0 \\
0 & -1\end{array}\right), \mu\right)$ \\
\hline 14 & $\left.\left(\begin{array}{ll}1 & 0 \\
0 & d\end{array}\right), \mu\right)$ \\
\hline 15 & \\
\hline 16 & $\left(\left(\begin{array}{ll}1 & 0 \\
0 & d\end{array}\right), 1\right)$ \\
\hline 17 & $\left(\left(\begin{array}{cc}a & 0 \\
c & a^{2}\end{array}\right), a\right)$ \\
\hline 18 & $\left(\left(\begin{array}{cc}a & 0 \\
0 & a^{2}\end{array}\right), 1\right)$ \\
\hline
\end{tabular}

Table 3. Automorphisms of kinematical Lie algebras (acting trivially on $\mathfrak{r}$ ). 


\subsection{Classification of kinematical Lie superalgebras}

We now proceed to analyse each kinematical Lie algebra $\mathfrak{k}$ in table 2 in turn and impose the Jacobi identity for the corresponding Lie superalgebras extending $\mathfrak{k}$. We recall that we are only interested in those Lie superalgebras where $[\boldsymbol{Q}, \boldsymbol{Q}] \neq 0$, so $c_{0}, \mathbb{C}_{1}, \mathbb{C}_{2}, \mathbb{C}_{3}$ cannot all simultaneously vanish.

\subsubsection{Kinematical Lie algebras without supersymmetric extensions}

There are three kinematical Lie algebras which cannot be extended to a kinematical superalgebra: $\mathfrak{s o}(4,1), \mathfrak{s o}(5)$ and the euclidean algebra (K7 in table 2$)$.

The euclidean algebra. From Lemma 1 we find that $\mathbb{p}=\mathbb{h}=0$ and $\mathfrak{b}^{2}=\frac{1}{4}$, so in particular $\mathbb{b} \in \mathbb{R}$, and from Lemma 2 we find that $\mathbb{C}_{2} \mathfrak{b}+\frac{1}{2} \mathbb{C}_{1}=0$. The $[H, \boldsymbol{Q}, \boldsymbol{Q}]$ component of the Jacobi identity shows that $\mathbb{C}_{2}=0$, so that also $\mathbb{C}_{1}=0$. The $[\boldsymbol{P}, \boldsymbol{Q}, \boldsymbol{Q}]$ component of the Jacobi identity is trivially satisfied, whereas the $[\boldsymbol{B}, \boldsymbol{Q}, \boldsymbol{Q}]$ component shows that $\mathbb{C}_{3}=0$ and also that $c_{0}=0$. In summary, there is no kinematical superalgebra extending the euclidean algebra for which $[\boldsymbol{Q}, \boldsymbol{Q}] \neq 0$; although there is a kinematical superalgebra where $[\mathrm{B}(\beta), \mathrm{Q}(s)]= \pm \frac{1}{2} \mathrm{Q}(\beta s)$, where both choices of sign are related by an automorphism of $\mathfrak{k}$ : e.g., time reversal $(\boldsymbol{J}, \boldsymbol{B}, \boldsymbol{P}, H) \mapsto(\boldsymbol{J},-\boldsymbol{B}, \boldsymbol{P},-H)$ or parity $(\boldsymbol{J}, \boldsymbol{B}, \boldsymbol{P}, H) \mapsto(\boldsymbol{J},-\boldsymbol{B},-\boldsymbol{P}, H)$.

$\mathfrak{s o}(\mathbf{4}, \mathbf{1})$. In this case, Lemma 1 gives that $\mathfrak{p}=\mathfrak{b}=0$, but then the $[\boldsymbol{B}, \boldsymbol{P}, \boldsymbol{Q}]$ component of the Jacobi identity cannot be satisfied, showing that the $\mathfrak{s o}(3)$ representation on the spinor module $S$ cannot be extended to a representation of $\mathfrak{s o}(4,1)$. The result would be different for $N=2$ extensions, since $\mathfrak{s o}(4,1) \cong \mathfrak{s p}(1,1)$ does have an irreducible spinorial representation of quaternionic dimension 2 .

$\mathfrak{s o ( 5 ) . ~ F r o m ~ L e m m a ~} 1$ we find from $[H, \boldsymbol{B}]=\boldsymbol{P}$ that $\mathbb{p}=[\mathfrak{b}, \mathfrak{h}]$ and, in particular, $p \in \operatorname{Im} \mathbb{H}$. But then $[\boldsymbol{P}, \boldsymbol{P}]=\boldsymbol{J}$ says that $\mathrm{p}^{2}=\frac{1}{4}$, so that in particular $\mathbb{p} \in \mathbb{R}$ and nonzero, which is a contradiction. Again this shows that the spinor representation $S$ of $\mathfrak{s o}(3)$ does not extend to a representation of $\mathfrak{s o}(5)$ and again the conclusion would be different for $N=2$ extensions, since $\mathfrak{s o}(5) \cong \mathfrak{s p}(2)$ does admit a quaternionic representation of quaternionic dimension 2 .

\subsubsection{Lorentzian kinematical superalgebras}

The Poincaré Lie algebra $(\mathrm{K} 8)$ and $\mathfrak{s o}(3,2)$ are lorentzian isometry Lie algebras: of Minkowski and anti de Sitter spacetimes, respectively. It is of course well known that such spacetimes admit $N=1$ superalgebras of maximal dimension. We treat them in this section for completeness.

The Poincaré superalgebra. From Lemma 1 we see that $p=\mathbb{h}=0$ and that $\mathbb{b}^{2}=-\frac{1}{4}$, so that in particular $\mathfrak{b} \in \operatorname{Im} \nVdash$. From Lemma 2 we see that $\frac{1}{2} \mathbb{C}_{1}+\mathbb{C}_{2} \mathfrak{b}=0$. The $[\boldsymbol{P}, \boldsymbol{Q}, \boldsymbol{Q}]$ component of the Jacobi identity is trivially satisfied, whereas the $[H, \boldsymbol{Q}, \boldsymbol{Q}]$ component forces $\mathbb{C}_{1}=\mathbb{C}_{2}=0$ and the $[\boldsymbol{B}, \boldsymbol{Q}, \boldsymbol{Q}]$ component says $\mathbb{C}_{3}=2 c_{0} \mathfrak{b}$. Demanding $[\boldsymbol{Q}, \boldsymbol{Q}] \neq 0$ requires $c_{0} \neq 0$. 
Using the quaternion automorphism, we can rotate $\mathbb{b}$ so that $\mathbb{b}=\frac{1}{2} \mathbb{k}$ and via the automorphism of the Poincaré Lie algebra which rescales $H$ and $P$ by the same amount, we can bring $c_{0}=1$. In summary, we have a unique isomorphism class of kinematical Lie superalgebras extending the Poincaré Lie algebra and consisting in the additional Lie brackets

$$
[\mathrm{B}(\beta), \mathrm{Q}(s)]=\frac{1}{2} \mathrm{Q}(\beta s \mathbb{k}) \quad \text { and } \quad[\mathrm{Q}(s), \mathrm{Q}(s)]=|s|^{2} H-\mathrm{P}(s k \bar{s}) .
$$

We will show below in section 3.3 that $\mathfrak{s}$ is isomorphic to the Poincaré superalgebra defined in the Introduction.

The AdS superalgebra. Here Lemma 1 and Lemma 2 give the following relations:

$$
\mathbb{P}=[\mathfrak{h}, \mathfrak{b}], \quad \mathbb{b}=[\mathfrak{p}, \mathfrak{h}], \quad \mathbb{h}=[\mathfrak{b}, \mathbb{p}], \quad \mathbb{b}^{2}=-\frac{1}{4}, \quad \mathbb{P}^{2}=-\frac{1}{4} \quad \text { and } \quad c_{0} \mathfrak{h}=\frac{1}{2} \mathbb{C}_{1}+\mathbb{C}_{2} \mathfrak{b}+\mathbb{C}_{3} \mathbb{P},
$$

and in addition $\mathbb{b} p+\mathfrak{p} b=0$, which simply states that $\mathbb{b} \perp \mathfrak{p}$. These relations imply that $\mathfrak{b}, \mathbb{p}, \mathbb{h} \in \operatorname{Im} \mathbb{H}$ and that $(2 \mathfrak{b}, 2 \mathbb{p}, 2 \mathfrak{h})$ is an oriented orthonormal basis for $\operatorname{Im} \mathbb{H}$. The remaining Jacobi identities give

$$
\mathbb{C}_{2}=-2 c_{0} \mathbb{P}, \quad \mathbb{C}_{3}=2 c_{0} \mathfrak{b} \Longrightarrow \mathbb{C}_{1}=-2 c_{0} \mathfrak{h},
$$

and some other relations which are identically satisfied. If $c_{0}=0$ then $[\boldsymbol{Q}, \boldsymbol{Q}]=0$, so we requires $c_{0} \neq 0$ and hence $\left(\frac{\mathbb{C}_{1}}{c_{0}}, \frac{\mathbb{C}_{2}}{c_{0}}, \frac{\mathbb{C}_{3}}{c_{0}}\right)$ defines a negatively oriented, orthonormal basis for

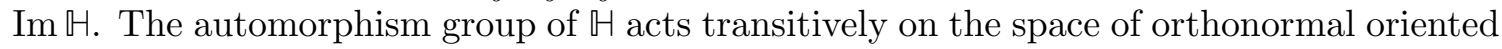
bases, so we can choose $(2 \mathfrak{b}, 2 \mathfrak{p}, 2 \mathfrak{h})=(\stackrel{\circ}{0}, \AA, \mathbb{j}, \mathbb{k})$ without loss of generality.

The resulting Lie superalgebra becomes

$$
\begin{aligned}
& {[H, \mathrm{Q}(s)]=\frac{1}{2} \mathrm{Q}(s \mathbb{k})} \\
& {[\mathrm{B}(\beta), \mathrm{Q}(s)]=\frac{1}{2} \mathrm{Q}(\beta s \stackrel{\circ}{)}} \\
& {[\mathrm{P}(\pi), \mathrm{Q}(s)]=\frac{1}{2} \mathrm{Q}(\pi s \text { j) }} \\
& {[\mathrm{Q}(s), \mathrm{Q}(s)]=c_{0}\left(|s|^{2} H+\mathrm{J}(s \llbracket \bar{s})+\mathrm{B}(s \rrbracket \bar{s})-\mathrm{P}(s \bar{\imath} \bar{s})\right) .}
\end{aligned}
$$

We may rescale $\boldsymbol{Q}$ to bring $c_{0}$ to a sign, but we can then change the sign via the automorphism of $\mathfrak{k}$ which sends $(\boldsymbol{J}, \boldsymbol{B}, \boldsymbol{P}, H) \mapsto(\boldsymbol{J}, \boldsymbol{P}, \boldsymbol{B},-H)$ and the inner automorphism

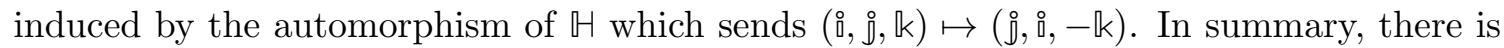
a unique kinematical Lie superalgebra with $[\boldsymbol{Q}, \boldsymbol{Q}] \neq 0$ extending $\mathfrak{k}=\mathfrak{s o}(3,2)$ : namely,

$$
\begin{aligned}
& {[H, \mathrm{Q}(s)]=\frac{1}{2} \mathrm{Q}(s \mathbb{k})} \\
& {[\mathrm{B}(\beta), \mathrm{Q}(s)]=\frac{1}{2} \mathrm{Q}(\beta s \stackrel{\circ}{)}} \\
& {[\mathrm{P}(\pi), \mathrm{Q}(s)]=\frac{1}{2} \mathrm{Q}(\pi s j)} \\
& {[\mathrm{Q}(s), \mathrm{Q}(s)]=|s|^{2} H+\mathrm{J}(s \llbracket \bar{s})+\mathrm{B}(s \rrbracket \bar{s})-\mathrm{P}(s ₫ \bar{s}) .}
\end{aligned}
$$

To show that this Lie superalgebra is isomorphic to $\mathfrak{o s p}(1 \mid 4)$ we may argue as follows. We first prove that $\mathfrak{s}_{\overline{0}}$ leaves invariant a symplectic form on $\mathfrak{s}_{\overline{1}}$. The most general rotationally invariant bilinear form on $\mathfrak{s}_{\overline{1}}$ is given by

$$
\omega\left(\mathrm{Q}\left(s_{1}\right), \mathrm{Q}\left(s_{2}\right)\right):=\operatorname{Re}\left(s_{1} \llbracket \bar{s}_{2}\right) \quad \text { for some } \mathbb{q} \in \mathbb{H} .
$$


Indeed, if $u \in \operatorname{Sp}(1)$ then

$$
\begin{aligned}
(u \cdot \omega)\left(\mathrm{Q}\left(s_{1}\right), \mathrm{Q}\left(s_{2}\right)\right) & =\omega\left(u^{-1} \cdot \mathrm{Q}\left(s_{1}\right), u^{-1} \cdot \mathrm{Q}\left(s_{2}\right)\right) \\
& =\omega\left(\mathrm{Q}\left(\bar{u} s_{1}\right), \mathrm{Q}\left(\bar{u} s_{2}\right)\right) \\
& =\operatorname{Re}\left(\bar{u} s_{1} \mathbb{\complement} \bar{s}_{2} u\right) \\
& =\operatorname{Re}\left(s_{1} \mathbb{\llbracket} \bar{s}_{2}\right) \\
& =\omega\left(\mathrm{Q}\left(s_{1}\right), \mathrm{Q}\left(s_{2}\right)\right) .
\end{aligned}
$$

Demanding that $\omega$ be invariant under the other generators $H, \boldsymbol{B}, \boldsymbol{P}$, we find that $\mathbb{q}=\mu \mathbb{k}$ for some $\mu \in \mathbb{R}$. Acting infinitesimally now,

$$
\begin{aligned}
(H \cdot \omega)\left(\mathrm{Q}\left(s_{1}\right), \mathrm{Q}\left(s_{2}\right)\right) & =-\omega\left(\left[H, \mathrm{Q}\left(s_{1}\right)\right], \mathrm{Q}\left(s_{2}\right)\right)-\omega\left(\mathrm{Q}\left(s_{1}\right),\left[H, \mathrm{Q}\left(s_{2}\right)\right]\right) \\
& =-\frac{1}{2} \omega\left(\mathrm{Q}\left(s_{1} \mathbb{k}\right), \mathrm{Q}\left(s_{2}\right)\right)-\frac{1}{2} \omega\left(\mathrm{Q}\left(s_{1}\right), \mathrm{Q}\left(s_{2} \mathbb{k}\right)\right) \\
& =-\frac{1}{2} \operatorname{Re}\left(s_{1} \mathbb{k} \mathbb{q} \bar{s}_{2}\right)+\frac{1}{2} \operatorname{Re}\left(s_{1} \mathbb{q} \mathbb{k} \bar{s}_{2}\right) \\
& =\frac{1}{2} \operatorname{Re}\left(s_{1}[\mathbb{q}, \mathbb{k}] \bar{s}_{2}\right),
\end{aligned}
$$

which must vanish for all $s_{1}, s_{2} \in S$, so that $[\mathfrak{q}, \mathbb{k}]=0$ and hence $\mathbb{q}=\lambda \mathbb{1}+\mu \mathbb{k}$ for some $\lambda, \mu \in \mathbb{R}$. A similar calculation with $\boldsymbol{B}$ and $\boldsymbol{P}$ shows that $q$ must anticommute with $\AA$ and $₫$ and thus $\mathbb{q}=\mu \mathbb{k}$. So the action of $\mathfrak{s}_{\overline{0}} \cong \mathfrak{s o}(3,2)$ on $\mathfrak{s}_{\overline{1}}$ defines a Lie algebra homomorphism $\mathfrak{s o}(3,2) \rightarrow \mathfrak{s p}(4, \mathbb{R})$, which is clearly nontrivial. Since $\mathfrak{s o}(3,2)$ is simple, it is injective and a dimension count shows that this is an isomorphism. But as representations of $\mathfrak{s o}(3,2), \odot^{2} \mathfrak{s}_{\overline{1}} \cong \wedge^{2} V$, where $V$ is the 5-dimensional vector representation of $\mathfrak{s}_{\overline{0}}$, and since $\wedge^{2} V \cong \mathfrak{s o}(V) \cong \mathfrak{s}_{\overline{0}}$ we have that there is one-dimensional space of $\mathfrak{s}_{\overline{0}}$-equivariant maps $\odot^{2} \mathfrak{s}_{\overline{1}} \rightarrow \mathfrak{s}_{\overline{0}}$. Since $[\boldsymbol{Q}, \boldsymbol{Q}] \neq 0$ the bracket $\odot^{2} \mathfrak{s}_{\overline{1}} \rightarrow \mathfrak{s}_{\overline{0}}$ is an isomorphism. This then shows that $\mathfrak{s}$ is, by definition, isomorphic to $\mathfrak{o} \mathfrak{s p}(1 \mid 4)$.

\subsubsection{The Carroll superalgebra}

For $\mathfrak{k}$ the Carroll Lie algebra (K6 in table 2), Lemma 1 implies that $\mathfrak{p}=\mathbb{b}=\mathfrak{h}=0$ and then Lemma 2 says that $\mathbb{C}_{1}=0$. The $[\boldsymbol{B}, \boldsymbol{Q}, \boldsymbol{Q}]$ Jacobi says that $\mathbb{C}_{3}=0$ and the $[\boldsymbol{P}, \boldsymbol{Q}, \boldsymbol{Q}]$ Jacobi says that $\mathbb{C}_{2}=0$. The only nonzero bracket involving $\boldsymbol{Q}$ is

$$
[\mathrm{Q}(s), \mathrm{Q}(s)]=c_{0}|s|^{2} H,
$$

which is nonzero for $c_{0} \neq 0$. If so, we can set $c_{0}=1$ via an automorphism of $\mathfrak{k}$ which rescales $H$ and $\boldsymbol{P}$, say, by $c_{0}$. In summary, there is a unique Carroll superalgebra with brackets

$$
[\mathrm{Q}(s), \mathrm{Q}(s)]=|s|^{2} H,
$$

in addition to those of the Carroll Lie algebra itself.

\subsubsection{The galilean superalgebras}

For $\mathfrak{k}$ the galilean Lie algebra ( $\mathrm{K} 2$ in table 2 ), Lemma 1 says that $\mathbb{b}=\mathfrak{p}=0$ and Lemma 2 says that $\mathbb{C}_{1}=2 c_{0} \mathfrak{h}$. The $[\boldsymbol{B}, \boldsymbol{Q}, \boldsymbol{Q}]$ Jacobi identity says that $\mathbb{C}_{1}=0$ and $c_{0}=0$. The 
$[\boldsymbol{P}, \boldsymbol{Q}, \boldsymbol{Q}]$ Jacobi identity is now identically satisfied, whereas the $[H, \boldsymbol{Q}, \boldsymbol{Q}]$ Jacobi identity gives

$$
\mathbb{h}_{2}+\mathbb{C}_{2} \overline{\mathrm{h}}=0 \quad \text { and } \quad \mathbb{C}_{2}+\mathbb{h}_{\mathbb{C}_{3}}+\mathbb{C}_{3} \overline{\mathrm{h}}=0 .
$$

Since $\mathbb{C}_{2}$ and $\mathbb{C}_{3}$ cannot both vanish, we see that this is only possible if $t h \in \operatorname{Im} H$ and hence these equations become $\left[\mathfrak{h}, \mathbb{C}_{2}\right]=0$ and $\mathbb{C}_{2}=\left[\mathbb{C}_{3}, \mathfrak{h}\right]$. There are two cases to consider, depending on whether or not $\mathbb{h}$ vanishes. If $\mathfrak{h}=0$, then $\mathbb{C}_{2}=0$ and $\mathbb{C}_{3}$ is arbitrary. If $\mathfrak{h} \neq 0$, then on the one hand $\mathbb{C}_{2}$ is collinear with $\mathbb{h}$, but also $\mathbb{C}_{2}=\left[\mathbb{C}_{3}, \mathfrak{h}\right]$, which means that $\mathbb{C}_{2}=0$ so that $\mathbb{C}_{3} \neq 0$ is collinear with $\mathbb{h}$. In either case, $\mathbb{C}_{3} \neq 0$ and $\mathbb{h}=\psi \mathbb{C}_{3}$, where $\psi \in \mathbb{R}$ can be zero.

This gives rise to the following additional brackets

$$
[H, \mathrm{Q}(s)]=\psi \mathrm{Q}\left(s \mathbb{C}_{3}\right) \quad \text { and } \quad[\mathrm{Q}(s), \mathrm{Q}(s)]=-\mathrm{P}\left(s \mathbb{C}_{3} \bar{s}\right)
$$

We may use the automorphisms of $\mathbb{H}$ to bring $\mathbb{C}_{3}=\phi \mathbb{k}$, for some nonzero $\phi \in \mathbb{R}$. We can set $\phi=1$ by an automorphism of $\mathfrak{k}$ which rescales $\boldsymbol{P}$ and also $\boldsymbol{B}$ and $H$ suitably. This still leaves the freedom to set $\psi=1$ if $\psi \neq 0$. In summary, we have two galilean superalgebras:

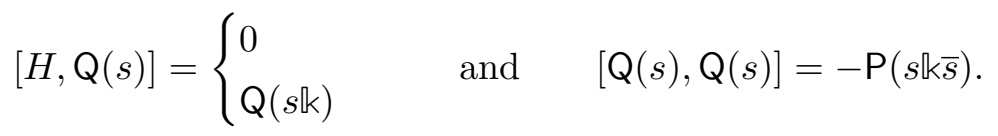

The first one (where $[H, \boldsymbol{Q}]=0$ ) is a contraction of the Poincaré superalgebra, whereas the second (where $[H, Q] \neq 0$ ) is not.

\subsubsection{Lie superalgebras associated with the static kinematical Lie algebra}

This is $\mathrm{K} 1$ in table 2. In this case, Lemma 1 says that $\mathbb{b}=\mathfrak{p}=0$ and Lemma 2 says that $\mathbb{C}_{1}=2 c_{0}$ h. The $[H, \boldsymbol{Q}, \boldsymbol{Q}]$ Jacobi identity says that $\mathbb{h} \in \operatorname{Im} \mathbb{H}$ and that $\left[\mathfrak{h}, \mathbb{C}_{i}\right]=0$ for $i=1,2,3$. Finally either the $[\boldsymbol{B}, \boldsymbol{Q}, \boldsymbol{Q}]$ or $[\boldsymbol{P}, \boldsymbol{Q}, \boldsymbol{Q}]$ Jacobi identities say that $\mathbb{C}_{1}=0$, so that $\mathrm{h} c_{0}=0$. This means that either $\mathrm{h}=0$ or else $c_{0}=0$ (or both).

There are several branches:

1. If $c_{0}=0$ and $\llbracket \neq 0, \mathbb{C}_{2}$ and $\mathbb{C}_{3}$ are collinear with $\mathfrak{h}$, but cannot both be zero. Using automorphisms of the static kinematical Lie algebra and the ability to rotate vectors, we can bring $\mathbb{h}=\frac{1}{2} \mathbb{k}, \mathbb{C}_{2}=0$ and $\mathbb{C}_{3}=\mathbb{k}$, so that we have a unique Lie superalgebra in this case, with additional brackets

$$
[H, \mathrm{Q}(s)]=\frac{1}{2} \mathrm{Q}(s \mathbb{k}) \quad \text { and } \quad[\mathrm{Q}(s), \mathrm{Q}(s)]=-\mathrm{P}(s \mathbb{k} \bar{s}) .
$$

2. If $c_{0}=0$ and $\mathbb{h}=0, \mathbb{C}_{2}$ and $\mathbb{C}_{3}$ are unconstrained, but not both zero. We distinguish two cases, depending on whether or not they are linearly independent:

(a) If they are linearly dependent, so that they are collinear, then we can use automorphisms to set $\mathbb{C}_{2}$, say, to zero and $\mathbb{C}_{3}=\mathbb{k}$. This results in the Lie superalgebra

$$
[\mathrm{Q}(s), \mathrm{Q}(s)]=-\mathrm{P}(s \mathbb{k} \bar{s}) .
$$


(b) If they are linearly independent, we can bring them to $\mathbb{C}_{2}=\AA$ and $\mathbb{C}_{3}=\mathbb{k}$, resulting in the Lie superalgebra

$$
[\mathrm{Q}(s), \mathrm{Q}(s)]=-\mathrm{B}(s \S \bar{s})-\mathrm{P}(s \llbracket \bar{s}) .
$$

3. Finally, if $c_{0} \neq 0$, then $\mathbb{h}=0$ and again $\mathbb{C}_{2}$ and $\mathbb{C}_{3}$ are unconstrained, but can now be zero. Moreover we can rescale $H$ so that $c_{0}=1$. We have three cases to consider, depending on whether they span a zero-, one- or two-dimensional real subspace of $\operatorname{Im} \amalg$ :

(a) If $\mathbb{C}_{2}=\mathbb{C}_{3}=0$ we have the Lie superalgebra

$$
[\mathrm{Q}(s), \mathrm{Q}(s)]=|s|^{2} H \text {. }
$$

(b) If $\mathbb{C}_{2}$ and $\mathbb{C}_{3}$ span a line, then we may use the automorphisms to set $\mathbb{C}_{2}=0$ and $\mathbb{C}_{3}=\mathbb{k}$, resulting in the Lie superalgebra

$$
[\mathrm{Q}(s), \mathrm{Q}(s)]=|s|^{2} H-\mathrm{P}(s \mathbb{k} \bar{s}) .
$$

(c) Finally, if $\mathbb{C}_{2}$ and $\mathbb{C}_{3}$ are linearly independent, we may use the automorphisms to set $\mathbb{C}_{2}=\AA$ and $\mathbb{C}_{3}=\mathbb{k}$, resulting in the Lie superalgebra

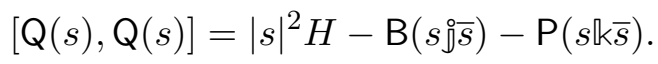

\subsubsection{Lie superalgebras associated with kinematical Lie algebra $\mathrm{K} 3_{\gamma}$}

Here Lemma 1 says that $\mathbb{b}=\mathfrak{p}=0$ and Lemma 2 says that $\mathbb{C}_{1}=2 c_{0}$ h. The $[\boldsymbol{B}, \boldsymbol{Q}, \boldsymbol{Q}]$ Jacobi identity says that $\mathbb{C}_{1}=0$ and $c_{0}=0$, whereas the $[\boldsymbol{P}, \boldsymbol{Q}, \boldsymbol{Q}]$ Jacobi identity offers no further conditions. Finally, the $[H, \boldsymbol{Q}, \boldsymbol{Q}]$ Jacobi identity gives two conditions

$$
\gamma \mathbb{C}_{2}=\mathfrak{h}_{\mathbb{C}_{2}}+\mathbb{C}_{2} \overline{\mathrm{h}} \quad \text { and } \quad \mathbb{C}_{3}=\mathfrak{h}_{\mathbb{C}_{3}}+\mathbb{C}_{3} \overline{\mathrm{h}},
$$

which are equivalent to

$$
(\gamma-2 \operatorname{Re} h) \mathbb{C}_{2}=\left[\operatorname{Im} \llbracket, \mathbb{C}_{2}\right] \quad \text { and } \quad(1-2 \operatorname{Re} \mathfrak{h}) \mathbb{C}_{3}=\left[\operatorname{Im} \llbracket, \mathbb{C}_{3}\right] .
$$

We see that we must distinguish two cases: $\gamma=1$ and $\gamma \in[-1,1)$.

If $\gamma \neq 1$, then we have two cases, depending on whether $\operatorname{Re} h=\frac{1}{2}$ or $\operatorname{Re} h=\frac{1}{2} \gamma$. In the former case, $\mathbb{C}_{2}=0$ and $\operatorname{Im} \mathbb{h}$ is collinear with $\mathbb{C}_{3} \neq 0$, whereas in the latter, $\mathbb{C}_{3}=0$ and $\operatorname{Im} \mathfrak{h}$ is collinear with $\mathbb{C}_{2} \neq 0$.

If $\gamma=1$, then $\operatorname{Re} \mathbb{h}=\frac{1}{2}$ and $\mathbb{C}_{2}, \operatorname{Im} \mathbb{h}$ and $\mathbb{C}_{3}$ are all collinear, with at least one of $\mathbb{C}_{2}$ and $\mathbb{C}_{3}$ nonzero. When $\gamma=1$, the automorphisms of $\mathbb{k}$ include the general linear group $\operatorname{GL}(2, \mathbb{R})$ acting on the two copies of the vector representation. Using this we can always assume that $\mathbb{C}_{2}=0$ and $\mathbb{C}_{3} \neq 0$.

In either case, all nonzero vectors are collinear and we can rotate them to lie along the $\mathbb{k}$ axis. In the case $\gamma=1$, we have a one-parameter family of Lie superalgebras:

$$
[H, \mathrm{Q}(s)]=\frac{1}{2} \mathrm{Q}(s(1+\lambda \mathbb{k})) \quad \text { and } \quad[\mathrm{Q}(s), \mathrm{Q}(s)]=-\mathrm{P}(s \mathbb{k} \bar{s}),
$$


where we have used the freedom to rescale $\boldsymbol{P}$ in order to set $\mathbb{C}_{3}=\mathbb{k}$. This is also a Lie superalgebra for $\gamma \neq 1$.

If $\gamma \neq 1$, we have an additional one-parameter family of Lie superalgebras:

$$
[H, \mathrm{Q}(s)]=\frac{1}{2} \mathrm{Q}(s(\gamma+\lambda \mathbb{k})) \quad \text { and } \quad[\mathrm{Q}(s), \mathrm{Q}(s)]=-\mathrm{B}(s \llbracket \bar{s})
$$

The parameter $\lambda$ is essential; that is, Lie superalgebras with different values of $\lambda$ are not isomorphic. One way to test this is the following. Let $[-,-]_{\lambda}$ denote the above Lie bracket. This satisfies the Jacobi identity for all $\lambda \in \mathbb{R}$. Write it as $[-,-]_{\lambda}=(1-\lambda)[-,-]_{0}+\lambda[-,-]_{1}$. The difference $[-,-]_{1}-[-,-]_{0}$ is a cocycle of the Lie superalgebra with $\lambda=0$. The parameter would be inessential if and only if it is a coboundary. One can check that this is not the case. This same argument shows that the parameters appearing in other Lie superalgebras are essential as well.

\subsubsection{Lie superalgebras associated with kinematical Lie algebra $\mathrm{K} 4 \chi$}

Here Lemma 1 says $\mathbb{b}=\mathbb{p}=0$ and Lemma 2 says that $\mathbb{c}_{1}=2 c_{0} \mathfrak{h}$. Then either the $[\boldsymbol{B}, \boldsymbol{Q}, \boldsymbol{Q}]$ or $[\boldsymbol{P}, \boldsymbol{Q}, \boldsymbol{Q}]$ Jacobi identities force $\mathbb{C}_{1}=0$ and $c_{0}=0$. The $[H, \boldsymbol{Q}, \boldsymbol{Q}]$ Jacobi identity results in the following two equations:

$$
\chi \mathbb{C}_{2}-\mathbb{C}_{3}=\mathbb{h}_{\mathbb{C}_{2}}+\mathbb{C}_{2} \overline{\mathrm{h}} \quad \text { and } \quad \chi \mathbb{c}_{3}+\mathbb{C}_{2}=\mathfrak{h}_{\mathbb{C}_{3}}+\mathbb{C}_{3} \overline{\mathrm{h}}
$$

or equivalently,

$$
(\chi-2 \operatorname{Re} \mathfrak{h}) \mathbb{C}_{2}-\mathbb{C}_{3}=\left[\operatorname{Im} \mathfrak{h}, \mathbb{C}_{2}\right] \quad \text { and } \quad(\chi-2 \operatorname{Re} \mathfrak{h}) \mathbb{C}_{3}+\mathbb{C}_{2}=\left[\operatorname{Im} \mathfrak{h}, \mathbb{C}_{3}\right] .
$$

Taking the inner product of the first equation with $\mathbb{C}_{2}$ and of the second equation with $\mathbb{C}_{3}$ and adding, we find

$$
(\chi-2 \operatorname{Re} h)\left(\left|\mathbb{C}_{2}\right|^{2}+\left|\mathbb{C}_{3}\right|^{2}\right)=0,
$$

and since $\mathbb{C}_{2}$ and $\mathbb{C}_{3}$ cannot both be zero, we see that $\operatorname{Re} \mathfrak{h}=\frac{\chi}{2}$, and hence that

$$
\left[\operatorname{Im} \llbracket, \mathbb{C}_{2}\right]=-\mathbb{C}_{3} \quad \text { and } \quad\left[\operatorname{Im} \llbracket, \mathbb{C}_{3}\right]=\mathbb{C}_{2},
$$

so that $\mathbb{C}_{3} \perp \mathbb{C}_{2}$. This shows that $\left(\operatorname{Im} \mathbb{h}, \mathbb{C}_{3}, \mathbb{C}_{2}\right)$ is an oriented orthogonal (but not necessarily orthonormal) basis. We can rotate them so that $\operatorname{Im} \mathbb{h}=\phi_{\mathfrak{j}}, \mathbb{C}_{3}=\psi k$ and $\mathbb{C}_{2}=2 \phi \psi$, but then we see that $\phi^{2}=\frac{1}{4}$. Using the automorphism of $\mathfrak{k}$ which rescales $\boldsymbol{B}$ and $\boldsymbol{P}$ simultaneously by the same amount we can assume that $\mathbb{C}_{3}=\mathbb{k}$ and hence if $\operatorname{Im} \mathbb{h}= \pm \frac{1}{2}$ § then $\mathbb{C}_{2}= \pm$. . But the two signs are related by the automorphism of $\mathbb{H}$ which sends

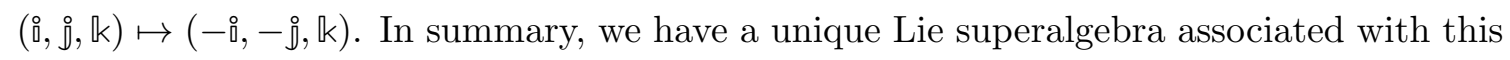
kinematical Lie algebra:

$$
[H, \mathrm{Q}(s)]=\frac{1}{2} \mathrm{Q}(s(\chi+\AA)) \quad \text { and } \quad[\mathrm{Q}(s), \mathrm{Q}(s)]=-\mathrm{B}(s \overline{0} \bar{s})-\mathrm{P}(s \llbracket \bar{s})
$$




\subsubsection{Lie superalgebras associated with kinematical Lie algebra K5}

Here Lemma 1 says that $\mathbb{b}=\mathfrak{p}=0$ and Lemma 2 says that $\mathbb{C}_{1}=2 c_{0}$ h. The $[\boldsymbol{B}, \boldsymbol{Q}, \boldsymbol{Q}]$ Jacobi identity forces $c_{0}=\mathbb{C}_{1}=0$, which then makes the $[\boldsymbol{P}, \boldsymbol{Q}, \boldsymbol{Q}]$ Jacobi identity be satisfied identically. The $[H, \boldsymbol{Q}, \boldsymbol{Q}]$ Jacobi identity gives two further equations

$$
\mathbb{C}_{2}=\mathfrak{h}_{2}+\mathbb{C}_{2} \overline{\mathbb{h}} \quad \text { and } \quad \mathbb{C}_{2}+\mathbb{C}_{3}=\mathfrak{R}_{\mathbb{C}_{3}}+\mathbb{C}_{3} \overline{\mathbb{h}} \text {. }
$$

The first equation is equivalent to

$$
(1-2 \operatorname{Re}(\mathfrak{h})) \mathbb{C}_{2}=\left[\operatorname{Im} \mathfrak{h}, \mathbb{C}_{2}\right] .
$$

If $\mathbb{C}_{2} \neq 0$, then $\operatorname{Re} \mathbb{h}=\frac{1}{2}$ and $\operatorname{Im} \mathbb{h}$ is collinear with $\mathbb{C}_{2}$. But then the second equation says that $\mathbb{C}_{2}=\left[\operatorname{Im} \mathbb{h}, \mathbb{C}_{3}\right]$, which is incompatible with $\mathbb{C}_{2}$ and $\operatorname{Im} \mathbb{h}$ being collinear. Therefore $\mathbb{C}_{2}=0$ and the second equation then says that $\operatorname{Re} \mathbb{h}=\frac{1}{2}$ and $\operatorname{Im} \mathbb{h}$ collinear with $\mathbb{C}_{3} \neq 0$. We have the following additional brackets

$$
[H, \mathrm{Q}(s)]=\frac{1}{2} \mathrm{Q}\left(s\left(1+\lambda \mathbb{C}_{3}\right)\right) \quad \text { and } \quad[\mathrm{Q}(s), \mathrm{Q}(s)]=-\mathrm{P}\left(s \mathbb{C}_{3} \bar{s}\right)
$$

where $\lambda \in \mathbb{R}$. We may rotate $\mathbb{C}_{3}$ to $\psi \mathbb{k}$, for some nonzero $\psi \in \mathbb{R}$. We can then rescale $\boldsymbol{P}$ and $\boldsymbol{B}$ simultaneously by the same amount to set $\psi=1$. In summary, we are left with the following one-parameter family of Lie superalgebras:

$$
[H, \mathrm{Q}(s)]=\frac{1}{2} \mathrm{Q}(s(1+\lambda \mathbb{k})) \quad \text { and } \quad[\mathrm{Q}(s), \mathrm{Q}(s)]=-\mathrm{P}(s \mathbb{k} \bar{s}) .
$$

As in the case of the Lie superalgebras associated with Lie algebra $\mathrm{K} 3_{\gamma}$, the parameter $\lambda$ is essential and Lie superalgebras with different values of $\lambda$ are not isomorphic.

\subsubsection{Lie superalgebras associated with kinematical Lie algebra K12}

Lemma 1 says that $\mathbb{b}^{2}=\frac{1}{2} \mathfrak{b}$, so that $\mathbb{b} \in \mathbb{R},[\mathfrak{h}, \mathbb{p}]=0$ and $\mathbb{p}^{2}=\frac{1}{2}\left(\mathbb{b}-\frac{1}{2}\right)$, so that $\mathbb{p} \in \operatorname{Im} \mathbb{H}$. (In particular, $\mathfrak{b} p=0$.) Lemma 2 does not simplify at this stage. The $[H, \boldsymbol{Q}, \boldsymbol{Q}]$ Jacobi identity says that $c_{0} \operatorname{Re} \mathbb{h}=0$ and that $\mathbb{h}_{i}+\mathbb{C}_{i} \overline{\mathbb{h}}=0$ for $i=1,2,3$. The $[\boldsymbol{B}, \boldsymbol{Q}, \boldsymbol{Q}]$ Jacobi identity says that $\mathfrak{b}_{\mathbb{C}_{1}}=0, \mathfrak{b}_{\mathbb{C}_{3}}=0$ and $\mathbb{C}_{1}=(2 \mathfrak{b}-1) \mathbb{c}_{2}$. Finally, the $[\boldsymbol{P}, \boldsymbol{Q}, \boldsymbol{Q}]$ Jacobi identity says that $c_{0} \mathrm{p}=0$, among other conditions that will turn out not to play a rôle.

We have two branches depending on the value of $\mathfrak{b}$ :

1. If $\mathbb{b}=0, \mathbb{p}^{2}=-\frac{1}{4}$, so that $c_{0}=0$. This means $\mathbb{C}_{1}+\mathbb{C}_{2}=0$ and $\mathbb{C}_{3}=2 \mathbb{C}_{1} \mathfrak{p}$ and none of $\mathbb{C}_{1,2,3}$ can vanish. This means that $\operatorname{Re} \mathbb{h}=0$ and that $\mathbb{h}$ and $\mathbb{C}_{i}$ are collinear for all $i=1,2,3$. Also $\mathbb{h}$ and $\mathbb{p}$ are collinear and this is inconsistent, unless $\mathbb{h}=0$ : indeed, if $\mathbb{p}$ and $\mathbb{C}_{i}$ are collinear with $\mathbb{h} \neq 0$, then $\mathbb{C}_{3}=2 \mathbb{C}_{1} \mathbb{p}$ cannot be satisfied, since the 1.h.s. is imaginary but the r.h.s. is real and both are nonzero. Therefore we conclude that $\mathbb{h}=0$. The condition $\mathbb{C}_{3}=2 \mathbb{C}_{1} \mathbb{P}$ says that there exists $\psi>0$ such that $\left(\psi^{-1} \mathbb{C}_{1}, 2 \mathfrak{p}, \psi^{-1} \mathbb{C}_{3}\right)$ is an oriented orthonormal basis, which can be rotated to $(\stackrel{0}{0}, \mathfrak{j}, \mathbb{k})$. In other words, we can write $\mathbb{C}_{1}=\psi_{0}^{\circ}, \mathbb{p}=\frac{1}{2} \AA$ and $\mathbb{C}_{3}=\psi \mathbb{k}$, so that $\mathbb{C}_{2}=-\psi$. . We

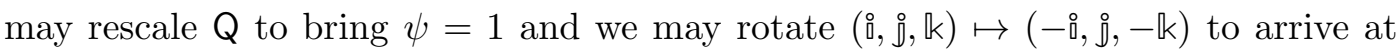
the following Lie superalgebra:

$$
[\mathrm{P}(\pi), \mathrm{Q}(s)]=\frac{1}{2} \mathrm{Q}(s \AA) \quad \text { and } \quad[\mathrm{Q}(s), \mathrm{Q}(s)]=\mathrm{J}(s \bar{\imath} \bar{s})-\mathrm{B}(s \bar{\imath} \bar{s})+\mathrm{P}(s k \bar{s}) .
$$


2. If $\mathbb{b}=\frac{1}{2}$, then $\mathbb{p}=0$ and also $\mathbb{C}_{1}=\mathbb{C}_{3}=0$ and $\mathbb{C}_{2}=2 c_{0} \mathfrak{h}$ with $c_{0} \neq 0$. We have two sub-branches, depending on whether or not $\mathbb{h}=0$.

(a) If $\llbracket$ h $=0$ we have the following Lie superalgebra, after rescaling $H$ to set $c_{0}=1$ :

$$
[\mathrm{B}(\beta), \mathrm{Q}(s)]=\frac{1}{2} \mathrm{Q}(\beta s) \quad \text { and } \quad[\mathrm{Q}(s), \mathrm{Q}(s)]=|s|^{2} H .
$$

(b) On the other hand, if $h \neq 0$, we may rotate it so that $2 h=\psi k$ for some $\psi$ such that $\psi c_{0}>0$. Then we may rescale $H$ and $\mathrm{Q}$ in such that a way that we bring $\psi c_{0}=1$, thus arriving at the following Lie superalgebra:

$$
[\mathrm{B}(\beta), \mathrm{Q}(s)]=\frac{1}{2} \mathrm{Q}(\beta s), \quad[H, \mathrm{Q}(s)]=\frac{1}{2} \mathrm{Q}(s \mathbb{k}) \text { and }[\mathrm{Q}(s), \mathrm{Q}(s)]=|s|^{2} H-\mathrm{B}(s \mathbb{k} \bar{s}) .
$$

\subsubsection{Lie superalgebras associated with kinematical Lie algebra K13}

Here Lemma 1 says that $\mathfrak{b}^{2}=\frac{1}{2} \mathfrak{b}$, so that $\mathfrak{b} \in \mathbb{R}$ and $\mathbb{p}^{2}=-\frac{1}{2}\left(\mathfrak{b}-\frac{1}{2}\right) \in \mathbb{R}$. Lemma 2 does not simplify further at this stage. The $[H, \boldsymbol{Q}, \boldsymbol{Q}]$ Jacobi identity says that $c_{0} \operatorname{Re} \mathbb{h}=0$ and $\mathbb{h}_{\mathbb{C}_{i}}+\mathbb{c}_{i} \overline{\mathrm{h}}=0$ for $i=1,2,3$. The $[\boldsymbol{B}, \boldsymbol{Q}, \boldsymbol{Q}]$ Jacobi identity says that $\mathrm{b}_{\mathbb{C}_{1}}=\mathrm{b}_{\mathbb{C}_{3}}=0$, whereas $\left(\mathbb{b}-\frac{1}{2}\right) \mathbb{C}_{2}=\frac{1}{2} \mathbb{C}_{1}$. Finally, the $[\boldsymbol{P}, \boldsymbol{Q}, \boldsymbol{Q}]$ Jacobi identity says that $\mathbb{C}_{1}=2 \mathbb{p}_{3}, \mathbb{C}_{3}=-2 \mathbb{p}_{2}$ and $\mathbb{C}_{3}=2 \mathrm{pc}_{1}$.

As usual we have two branches depending on the value of $\mathfrak{b}$ :

1. If $\mathfrak{b}=0$, then $\mathbb{p}^{2}=\frac{1}{4}$. Due to the automorphism of $\mathfrak{k}$ which changes the sign of $\boldsymbol{P}$, we may assume $\mathbb{p}=\frac{1}{2}$ without loss of generality. It follows that $\mathbb{C}_{1}=c_{0} \mathbb{R}$ and that $\mathbb{C}_{2}=-\mathbb{C}_{1}=-c_{0}$ th and that $\mathbb{C}_{3}=\mathbb{C}_{1}=c_{0}$ h. If $c_{0}=0$ then $\mathbb{C}_{i}=0$ for all $i$, so we must have $c_{0} \neq 0$. In that case, $\mathbb{h} \in \operatorname{Im} \mathbb{H}$ and $\llbracket$ is collinear with all $\mathbb{C}_{i}$ for $i=1,2,3$. We distinguish two cases, depending on whether or not $\mathbb{h}=0$ :

(a) If $\mathbb{h} \neq 0$, we may rotate it so that $\mathbb{h}=\psi k$ where $\psi c_{0}>0$. We may rescale $H \mapsto \psi^{-1} H$ (which is an automorphism of $\mathfrak{k}$ ) and rescale $\boldsymbol{Q}$ to bring $\psi c_{0}=1$. In summary, we arrive at the following Lie superalgebra:

$$
\begin{aligned}
{[H, \mathrm{Q}(s)] } & =\mathrm{Q}(s \mathbb{k}), \quad[\mathrm{P}(\pi), \mathrm{Q}(s)]=\frac{1}{2} \mathrm{Q}(\pi s) \quad \text { and } \\
{[\mathrm{Q}(s), \mathrm{Q}(s)] } & =|s|^{2} H-\mathrm{J}(s \mathbb{k} \bar{s})+\mathrm{B}(s \mathbb{k})-\mathrm{P}(s \mathbb{k} \bar{s}) .
\end{aligned}
$$

(b) If $\mathrm{t}=0$, then we have the Lie superalgebra

$$
[\mathrm{P}(\pi), \mathrm{Q}(s)]=\frac{1}{2} \mathrm{Q}(\pi s) \quad \text { and } \quad[\mathrm{Q}(s), \mathrm{Q}(s)]=|s|^{2} H .
$$

2. If $\mathbb{b}=\frac{1}{2}$, then $\mathbb{p}=0$ and $\mathbb{C}_{1}=\mathbb{C}_{3}=0$ with $\mathbb{C}_{2}=2 c_{0}$ th with $c_{0} \neq 0$ and $\mathbb{h} \in \operatorname{Im} \mathbb{H}$. Again we distinguish between vanishing and nonvanishing th:

(a) If $\mathfrak{h} \neq 0$, we may rotate it so that $2 h=\psi \mathbb{k}$ with $\psi c_{0}>0$. We apply the $\mathfrak{k}$ automorphism $H \mapsto \psi^{-1} H$ and rescale $\boldsymbol{Q}$ to bring $\psi c_{0}=1$, thus resulting in the Lie superalgebra

$$
[H, \mathrm{Q}(s)]=\frac{1}{2} \mathrm{Q}(s \mathbb{k}), \quad[\mathrm{B}(\beta), \mathrm{Q}(s)]=\frac{1}{2} \mathrm{Q}(\beta s) \text { and }[\mathrm{Q}(s), \mathrm{Q}(s)]=|s|^{2} H-\mathrm{B}(s \mathbb{k} \bar{s}) .
$$


(b) If $\mathrm{h}=0$, we arrive at the Lie superalgebra

$$
[\mathrm{B}(\beta), \mathrm{Q}(s)]=\frac{1}{2} \mathrm{Q}(\beta s) \quad \text { and } \quad[\mathrm{Q}(s), \mathrm{Q}(s)]=|s|^{2} H .
$$

\subsubsection{Lie superalgebras associated with kinematical Lie algebra K14}

Here Lemma 1 says that $\mathfrak{p}=0$ and $2 \mathfrak{b}^{2}=\mathfrak{b}$, so that $\mathfrak{b} \in \mathbb{R}$. Lemma 2 says that $\frac{1}{2} \mathbb{C}_{1}+\mathbb{C}_{2} \mathfrak{b}=$ $c_{0}$ h. The $[\boldsymbol{P}, \boldsymbol{Q}, \boldsymbol{Q}]$ Jacobi identity says that $\mathbb{C}_{1}=0$, so that $c_{0}$ h $=\mathbb{C}_{2} \mathfrak{b}$. The $[\boldsymbol{B}, \boldsymbol{Q}, \boldsymbol{Q}]$ Jacobi identity says that $(2 \mathfrak{b}-1) \mathbb{C}_{2}=0$ and $\mathfrak{b}_{3}=0$, whereas the $[\boldsymbol{B}, \boldsymbol{Q}, \boldsymbol{Q}]$ Jacobi identity says that $\mathbb{h}_{i}+\mathbb{C}_{i} \overline{\mathrm{h}}=0$ for $i=2,3$.

We have two branches, depending on the value of $\mathfrak{b}$ :

1. If $\mathbb{b}=0$ then $\mathbb{C}_{2}=0$ and we have two sub-branches depending on whether or not $c_{0}=0$ :

(a) If $c_{0}=0$ then $\mathbb{C}_{3} \neq 0$, so that $\operatorname{Re} \mathbb{h}=0$ and $\mathbb{h}$ is collinear with $\mathbb{C}_{3}$. We may rotate $\mathbb{C}_{3}$ to lie along $\mathbb{k}$, say, and then use automorphisms of $\mathfrak{k}$ to set $\mathbb{C}_{3}=\mathbb{k}$. If $\mathbb{h} \neq 0$, we may also set it equal to $\mathbb{k}$. In summary, we have two isomorphism classes of Lie superalgebras here:

$$
[H, \mathrm{Q}(s)]=\left\{\begin{array}{l}
0 \\
\mathrm{Q}(s \mathbb{k})
\end{array} \quad \text { and } \quad[\mathrm{Q}(s), \mathrm{Q}(s)]=-\mathrm{P}(s k \bar{s}) .\right.
$$

(b) If $c_{0} \neq 0$, then $\mathbb{h}=0$ and $\mathbb{C}_{3}$ is free: if nonzero we may rotate it to $\mathbb{k}$ and rescaling $\boldsymbol{P}$, which is an automorphism of $\mathfrak{k}$, we can bring it to $\mathbb{k}$. Rescaling $H$ we can bring $c_{0}=1$. This gives two isomorphism classes of Lie superalgebras:

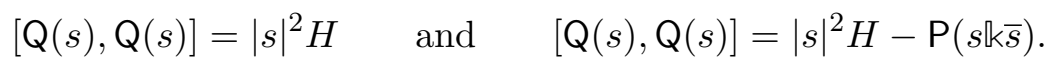

2. If $\mathfrak{b}=\frac{1}{2}$, then $\mathbb{C}_{3}=0$ and $\mathbb{C}_{2}=2 c_{0} \mathfrak{h}$, and we have two cases, depending on whether or not $\mathbb{h}=0$.

(a) If $\mathrm{h}=0$ then $\mathbb{C}_{2}=0$, and then $c_{0} \neq 0$. Rescaling $H$ we can set $c_{0}=1$ and we arrive at the Lie superalgebra

$$
[\mathrm{B}(\beta), \mathrm{Q}(s)]=\frac{1}{2} \mathrm{Q}(\beta s) \quad \text { and } \quad[\mathrm{Q}(s), \mathrm{Q}(s)]=|s|^{2} H .
$$

(b) If $\mathfrak{h} \neq 0$ we can rotate and rescale $\boldsymbol{Q}$ such that $\mathbb{C}_{2}=2 c_{0} \mathfrak{h}=\mathbb{k}$ and then we can rescale $H$ so that $c_{0}=1$. The resulting Lie superalgebra is now

$$
[H, \mathrm{Q}(s)]=\frac{1}{2} \mathrm{Q}(s \mathbb{k}), \quad[\mathrm{B}(\beta), \mathrm{Q}(s)]=\frac{1}{2} \mathrm{Q}(\beta s) \text { and }[\mathrm{Q}(s), \mathrm{Q}(s)]=|s|^{2} H-\mathrm{B}(s \mathbb{k} \bar{s}) \text {. }
$$

\subsubsection{Lie superalgebras associated with kinematical Lie algebra K15}

Here Lemma 1 says that $\mathbb{b}=\mathfrak{p}=0$, whereas Lemma 2 says that $\mathbb{C}_{1}=2 c_{0} \mathfrak{h}$. The $[\boldsymbol{B}, \boldsymbol{Q}, \boldsymbol{Q}]$ Jacobi identity says that $\mathbb{C}_{1}=\mathbb{C}_{2}=0$, and hence the $[\boldsymbol{P}, \boldsymbol{Q}, \boldsymbol{Q}]$ component is identically satisfied. Finally, the $[H, \boldsymbol{Q}, \boldsymbol{Q}]$ Jacobi identity says that $\mathbb{h}_{3}+\mathbb{C}_{3} \overline{\mathrm{h}}=0$, which expands to

$$
2 \operatorname{Re}(\mathfrak{h}) \mathbb{C}_{3}+\left[\operatorname{Im} \mathfrak{h}, \mathbb{C}_{3}\right]=0 .
$$

We have two branches of solutions: 
1. If $c_{0}=0$, then $\mathbb{C}_{3} \neq 0$ and hence $\operatorname{Re} \mathbb{h}=0$ and $\mathbb{h}$ is collinear with $\mathbb{C}_{3}$. We may rotate $\mathbb{C}_{3}$ to lie along $\mathbb{k}$ and then rescale $\boldsymbol{Q}$ so that $\mathbb{C}_{3}=\mathbb{k}$. If $\mathbb{h} \neq 0$, we may use automorphisms of $\mathfrak{k}$ to set $\mathbb{h}=\mathbb{k}$ as well. In summary, we have two isomorphism classes of Lie superalgebras:

$$
[H, \mathrm{Q}(s)]=\left\{\begin{array}{l}
\mathrm{Q}(s \mathbb{k}) \\
0
\end{array} \quad \text { and } \quad[\mathrm{Q}(s), \mathrm{Q}(s)]=-\mathrm{P}(s \mathbb{k} \bar{s}) .\right.
$$

2. If $c_{0} \neq 0$, then $\mathbb{h}=0$ and $\mathbb{C}_{3}$ is unconstrained. If nonzero, we may rotate it to lie along $\mathbb{k}$, rescale $\boldsymbol{Q}$ so that $\mathbb{C}_{3}=\mathbb{k}$ and then use automorphisms of $\mathfrak{k}$ to set $c_{0}=1$. In summary, we have two isomorphism classes of Lie superalgebras:

$$
[\mathrm{Q}(s), \mathrm{Q}(s)]=|s|^{2} H \quad \text { or } \quad[\mathrm{Q}(s), \mathrm{Q}(s)]=|s|^{2} H-\mathrm{P}(s \mathbb{k} \bar{s}) .
$$

\subsubsection{Lie superalgebras associated with kinematical Lie algebra K16}

Here Lemma 1 says that $\mathbb{p}=0$ and $\mathfrak{b}\left(\mathfrak{b}-\frac{1}{2}\right)=0$, so that $\mathbb{b} \in \mathbb{R}$. Lemma 2 then says that $c_{0} \mathfrak{h}=\frac{1}{2} \mathbb{C}_{1}+\mathbb{C}_{2} \mathfrak{b}$. Now the $[\boldsymbol{P}, \boldsymbol{Q}, \boldsymbol{Q}]$ Jacobi identity says that $c_{0}=0$ and $\mathbb{C}_{1}=0$, so that $\mathbb{C}_{2} \mathfrak{b}=0$. The $[H, \boldsymbol{Q}, \boldsymbol{Q}]$ Jacobi identity says that $\mathbb{h}_{\mathbb{C}_{2}}+\mathbb{C}_{2} \overline{\mathrm{h}}=0$ and $\mathbb{h}_{\mathbb{C}_{3}}+\mathbb{C}_{3} \overline{\mathrm{h}}=\mathbb{C}_{3}$. Finally the $[\boldsymbol{B}, \boldsymbol{Q}, \boldsymbol{Q}]$ Jacobi identity says that $\mathbb{b}_{\mathbb{C}_{3}}=0$ and $\left(\mathfrak{b}-\frac{1}{2}\right) \mathbb{C}_{2}=0$.

Notice that if $\mathbb{b}=\frac{1}{2}$ then $\mathbb{C}_{3}=0$ and $\mathbb{C}_{2}=0$, contradicting $[\boldsymbol{Q}, \boldsymbol{Q}] \neq 0$, so we must have $\mathbb{b}=0$. Now $\mathbb{C}_{2}=0$ and hence $\mathbb{C}_{3} \neq 0$. It then follows that $\operatorname{Re} \mathbb{h}=\frac{1}{2}$ and $\operatorname{Im} \mathbb{h}$ is collinear with $\mathbb{C}_{3}$. We can rescale $\boldsymbol{P}$ (which is an automorphism of $\mathfrak{k}$ ) and rotate so that $\mathbb{C}_{3}=\mathbb{k}$, so that $\mathbb{h}=\frac{1}{2}(1+\lambda \mathbb{k})$ for $\lambda \in \mathbb{R}$. The resulting one-parameter family of Lie superalgebras is then

$$
[H, \mathrm{Q}(s)]=\frac{1}{2} \mathrm{Q}(s(1+\lambda \mathbb{k})) \quad \text { and } \quad[\mathrm{Q}(s), \mathrm{Q}(s)]=-\mathrm{P}(s \mathbb{k} \bar{s}) .
$$

As in the case of the Lie superalgebras associated with Lie algebras $\mathrm{K} 3_{\gamma}$ and $\mathrm{K} 5$, the parameter $\lambda$ is essential and Lie superalgebras with different values of $\lambda$ are not isomorphic.

\subsubsection{Lie superalgebras associated with kinematical Lie algebra K17}

Here Lemma 1 simply sets $\mathbb{b}=\mathfrak{p}=0$ and Lemma 2 says $\mathbb{C}_{1}=2 c_{0} \mathfrak{t}$. The $[\boldsymbol{P}, \boldsymbol{Q}, \boldsymbol{Q}]$ Jacobi identity sets $\mathbb{C}_{1}=0$ and hence $c_{0} \mathrm{~h}=0$. The $[\boldsymbol{B}, \boldsymbol{Q}, \boldsymbol{Q}]$ Jacobi identity sets $c_{0}=0$ and $\mathbb{C}_{2}=0$, whereas the $[H, \boldsymbol{Q}, \boldsymbol{Q}]$ Jacobi identity says that $\mathbb{t}$ is collinear with $\mathbb{C}_{3} \neq 0$. We can rotate $\mathbb{C}_{3}$ to lie along $\mathbb{k}$ and rescale $Q$ to effectively set it to $\mathbb{k}$. Then $\mathbb{h}=\frac{\psi}{2} \mathbb{k}$ for some $\psi$ and rescaling $H$ allows us to set $\psi=1$. In summary, we have a unique Lie superalgebra associated with this kinematical Lie algebra: namely,

$$
[H, \mathrm{Q}(s)]=\frac{1}{2} \mathrm{Q}(s \mathbb{k}) \quad \text { and } \quad[\mathrm{Q}(s), \mathrm{Q}(s)]=-\mathrm{P}(s k \bar{s}) .
$$

\subsubsection{Lie superalgebras associated with kinematical Lie algebra K18}

Here Lemma 1 simply sets $\mathbb{b}=\mathfrak{p}=0$ and Lemma 2 says $\mathbb{C}_{1}=2 c_{0}$ h. The $[\boldsymbol{P}, \boldsymbol{Q}, \boldsymbol{Q}]$ Jacobi identity sets $\mathbb{C}_{1}=0$ and $c_{0}=0$, whereas the $[\boldsymbol{B}, \boldsymbol{Q}, \boldsymbol{Q}]$ Jacobi identity sets $\mathbb{C}_{2}=0$. Finally, the $[H, \boldsymbol{Q}, \boldsymbol{Q}]$ Jacobi identity says that $\operatorname{Re} \mathbb{h}=1$ and $\operatorname{Im} \mathfrak{h}=\lambda \mathbb{C}_{3}$ for some $\lambda \in \mathbb{R}$. We can rotate $\mathbb{C}_{3}$ to lie along $\mathbb{k}$ and rescale $Q$ to effectively set it to $\mathbb{k}$. Then $\mathbb{h}=1+\lambda \mathbb{k}$. In summary, 
we have a one-parameter family of Lie superalgebras associated with this kinematical Lie algebra: namely,

$$
[H, \mathrm{Q}(s)]=\mathrm{Q}(s(1+\lambda \mathbb{k})) \quad \text { and } \quad[\mathrm{Q}(s), \mathrm{Q}(s)]=-\mathrm{P}(s \llbracket \bar{s}) .
$$

As in the case of the Lie superalgebras associated with Lie algebras $\mathrm{K} 3_{\gamma}, \mathrm{K} 5$ and $\mathrm{K} 16$, the parameter $\lambda$ is essential and Lie superalgebras with different values of $\lambda$ are not isomorphic.

\subsubsection{Summary}

Table 4 summarises the results. In that table we list the isomorphism classes of kinematical Lie superalgebras (with $[\boldsymbol{Q}, \boldsymbol{Q}] \neq 0$ ). Recall that the Lie brackets involving $\boldsymbol{Q}$ are the $[\boldsymbol{Q}, \boldsymbol{Q}]$ bracket and also

$$
[H, \mathrm{Q}(s)]=\mathrm{Q}(s \mathfrak{h}), \quad[\mathrm{B}(\beta), \mathrm{Q}(s)]=\mathrm{Q}(\beta s \mathrm{~b}), \quad[\mathrm{P}(\pi), \mathrm{Q}(s)]=\mathrm{Q}(\pi s \mathrm{p}),
$$

for some $\mathfrak{h}, \mathfrak{b}, \mathfrak{p} \in \mathbb{H}$. In table 4 we list any nonzero values of $\mathfrak{h}, \mathfrak{b}, \mathfrak{p}$ and the $[\boldsymbol{Q}, \boldsymbol{Q}]$ bracket. The first column is simply the label for the Lie superalgebra, the second column is the corresponding kinematical Lie algebra, the next columns are $\mathbb{h}, \mathbb{b}, \mathbb{p}$ and $[\boldsymbol{Q}, \boldsymbol{Q}]$. The next four columns are the possible $\mathfrak{s o}(3)$-equivariant $\mathbb{Z}$-gradings (with $\boldsymbol{J}$ of degree 0 ) compatible with the $\mathbb{Z}_{2}$-grading; that is, such that the parity is the reduction modulo 2 of the degree. This requires, in particular, that $q$ be an odd integer, which we can take to be -1 by convention, if so desired.

\subsection{Classification of aristotelian Lie superalgebras}

Table 5 lists the aristotelian Lie algebras (with three-dimensional space isotropy), classified in ([4], appendix A). In this section, we classify the $N=1$ supersymmetric extensions of the aristotelian Lie algebras (with $[\boldsymbol{Q}, \boldsymbol{Q}] \neq 0$ ).

\subsubsection{Lie superalgebras associated with aristotelian Lie algebra A1}

We start with the static aristotelian Lie algebra A1, whose only nonzero brackets are $[\boldsymbol{J}, \boldsymbol{J}]=\boldsymbol{J}$ and $[\boldsymbol{J}, \boldsymbol{P}]=\boldsymbol{P}$. Any supersymmetric extension $\mathfrak{g}$ has possible brackets

$[H, \mathrm{Q}(s)]=\mathrm{Q}(s \rrbracket), \quad[\mathrm{P}(\pi), \mathrm{Q}(s)]=\mathrm{Q}(\pi s \mathbb{P})$ and $[\mathrm{Q}(s), \mathrm{Q}(s)]=c_{0}|s|^{2} H-\mathrm{J}\left(s \mathbb{C}_{1} \bar{s}\right)-\mathrm{P}\left(s \mathbb{C}_{3} \bar{s}\right)$

for some $\mathbb{h}, \mathbb{p} \in \mathbb{H}, c_{0} \in \mathbb{R}$ and $\mathbb{C}_{1}, \mathbb{C}_{3} \in \operatorname{Im} \mathbb{H}$, using the same notation as in section 3 . We can reuse Lemmas 1 and 2 , by setting $b=0$ and $\mathbb{C}_{2}=0$ and ignoring $\boldsymbol{B}$. Doing so we find that $\mathbb{p}=0$ and that $\mathbb{C}_{1}=2 c_{0}$ h. The $[H, \boldsymbol{Q}, \boldsymbol{Q}]$ component of the Jacobi identity gives $c_{0} \operatorname{Re} \mathbb{h}=0$ (which already follows from Lemma 2), $\mathbb{c}_{1} \overline{\mathbb{h}}+\mathfrak{h}_{\mathbb{C}_{1}}=0$ and $\mathbb{C}_{3} \overline{\mathfrak{h}}+\mathbb{h}_{\mathbb{C}_{3}}=0$. The $[\boldsymbol{P}, \boldsymbol{Q}, \boldsymbol{Q}]$ component of the Jacobi identity says that $\left[s \mathbb{C}_{1} \bar{s}, \pi\right]=0$ for all $\pi \in \operatorname{Im} \mathbb{H}$ and $s \in \mathbb{H}$, which says $\mathbb{C}_{1}=0$ and hence $c_{0} \mathbb{h}=0$. This gives rise to two branches:

1. If $c_{0}=0$, then $\mathbb{C}_{3} \neq 0$ and the condition $\mathbb{C}_{3} \overline{\mathfrak{h}}+\mathbb{h}_{\mathbb{C}_{3}}=0$ is equivalent to $\left[\operatorname{Im} \mathfrak{h}, \mathbb{C}_{3}\right]=$ $-2 \mathbb{C}_{3} \operatorname{Re} \mathfrak{h}$, which says Re $\mathfrak{h}=0$ and hence that $\mathbb{h}$ and $\mathbb{C}_{3}$ are collinear. We can change 


\begin{tabular}{|c|c|c|c|c|c|c|c|c|c|}
\hline $\mathrm{S} \#$ & $\mathfrak{k}$ & Th & b & $p$ & {$[\mathrm{Q}(s), \mathrm{Q}(s)]$} & $w_{H}$ & $w_{B}$ & $w_{\boldsymbol{P}}$ & $w_{Q}$ \\
\hline 1 & K1 & $\frac{1}{2} \mathbb{k}$ & & & $-\mathrm{P}(s \mathbb{k} \bar{s})$ & 0 & $2 m$ & $2 q$ & $q$ \\
\hline 2 & K1 & & & & $|s|^{2} H-\mathrm{B}\left(s s^{\circ} \bar{s}\right)-\mathrm{P}(s \mathbb{k} \bar{s})$ & $2 q$ & $2 q$ & $2 q$ & $q$ \\
\hline 3 & K1 & & & & $|s|^{2} H-\mathrm{P}(s \mathbb{k} \bar{s})$ & $2 q$ & $2 m$ & $2 q$ & $q$ \\
\hline 4 & K1 & & & & $|s|^{2} H$ & $2 q$ & $2 m$ & $2 p$ & $q$ \\
\hline 5 & $\mathrm{~K} 1$ & & & & $-\mathrm{B}(s \oint \bar{s})-\mathrm{P}(s \llbracket \bar{k})$ & $2 n$ & $2 q$ & $2 q$ & $q$ \\
\hline 6 & K1 & & & & $-\mathrm{P}(s \mathbb{k} \bar{s})$ & $2 n$ & $2 m$ & $2 q$ & $q$ \\
\hline 7 & $\mathrm{~K} 2$ & $\mathbb{k}$ & & & $-\mathrm{P}(s \mathbb{k} \bar{s})$ & 0 & $2 q$ & $2 q$ & $q$ \\
\hline 8 & $\mathrm{~K} 2$ & & & & $-\mathrm{P}(s \mathbb{k} \bar{s})$ & $2 n$ & $2(q-n)$ & $2 q$ & $q$ \\
\hline $9_{\gamma \in[-1,1], \lambda \in \mathbb{R}}$ & $\mathrm{K} 3_{\gamma}$ & $\frac{1}{2}(1+\lambda \mathbb{k})$ & & & $-\mathrm{P}(s \mathbb{k} \bar{s})$ & 0 & $2 m$ & $2 q$ & $q$ \\
\hline $10_{\gamma \in[-1,1), \lambda \in \mathbb{R}}$ & $\mathrm{K} 3_{\gamma}$ & $\frac{1}{2}(\gamma+\lambda \mathbb{k})$ & & & $-\mathrm{B}(s \mathbb{k} \bar{s})$ & 0 & $2 q$ & $2 p$ & $q$ \\
\hline $11_{\chi \geq 0}$ & $\mathrm{~K} 4 \chi$ & $\frac{1}{2}(\chi+\jmath)$ & & & $-\mathrm{B}(s \stackrel{\circ}{\bar{s}})-\mathrm{P}(s \llbracket \bar{s})$ & 0 & $2 q$ & $2 q$ & $q$ \\
\hline $12_{\lambda \in \mathbb{R}}$ & K5 & $\frac{1}{2}(1+\lambda \mathbb{k})$ & & & $-\mathrm{P}(s \mathbb{k} \bar{s})$ & 0 & $2 q$ & $2 q$ & $q$ \\
\hline 13 & K6 & & & & $|s|^{2} H$ & $2 q$ & $2 m$ & $2(q-m)$ & $q$ \\
\hline 14 & K8 & & $\frac{1}{2} \mathbb{k}$ & & $|s|^{2} H-\mathrm{P}(s k \bar{s})$ & $2 q$ & 0 & $2 q$ & $q$ \\
\hline 15 & K11 & $\frac{1}{2} \mathbb{k}$ & $\frac{1}{2} \circ$ & $\frac{1}{2} §$ & $|s|^{2} H+\mathrm{J}(s \mathbb{k} \bar{s})+\mathrm{B}\left(s \_\bar{s}\right)-\mathrm{P}\left(s_{\triangleright}^{\circ} \bar{s}\right)$ & - & - & - & - \\
\hline 16 & $\mathrm{~K} 12$ & & & $\frac{1}{2} \risingdotseq$ & $\mathrm{J}\left(s s^{\circ} \bar{s}\right)-\mathrm{B}(s \overline{0} \bar{s})+\mathrm{P}(s \llbracket \bar{s})$ & - & - & - & - \\
\hline 17 & $\mathrm{~K} 12$ & & $\frac{1}{2}$ & & $|s|^{2} H$ & $2 q$ & 0 & 0 & $q$ \\
\hline 18 & $\mathrm{~K} 12$ & $\frac{1}{2} \mathbb{k}$ & $\frac{1}{2}$ & & $|s|^{2} H-\mathrm{B}(s \mathbb{k} \bar{s})$ & - & - & - & - \\
\hline 19 & K13 & $\mathbb{k}$ & & $\frac{1}{2}$ & $|s|^{2} H-\mathrm{J}(s \mathbb{k} \bar{s})+\mathrm{B}(s \mathbb{k} \bar{s})-\mathrm{P}(s \mathbb{k} \bar{s})$ & - & - & - & - \\
\hline 20 & K13 & & & $\frac{1}{2}$ & $|s|^{2} H$ & $2 q$ & 0 & 0 & $q$ \\
\hline 21 & K13 & & $\frac{1}{2}$ & & $|s|^{2} H$ & $2 q$ & 0 & 0 & $q$ \\
\hline 22 & K13 & $\frac{1}{2} \mathbb{k}$ & $\frac{1}{2}$ & & $|s|^{2} H-\mathrm{B}(s \llbracket \bar{s})$ & - & - & - & - \\
\hline 23 & K14 & $\mathbb{k}$ & & & $-\mathrm{P}(s \mathbb{k} \bar{s})$ & 0 & 0 & $2 q$ & $q$ \\
\hline 24 & K14 & & & & $-\mathrm{P}(s \mathbb{k} \bar{s})$ & $2 n$ & 0 & $2 q$ & $q$ \\
\hline 25 & K14 & & & & $|s|^{2} H$ & $2 q$ & 0 & $2 p$ & $q$ \\
\hline 26 & K14 & & & & $|s|^{2} H-\mathrm{P}(s \mathbb{k} \bar{s})$ & $2 q$ & 0 & $2 q$ & $q$ \\
\hline 27 & $\mathrm{~K} 14$ & & $\frac{1}{2}$ & & $|s|^{2} H$ & $2 q$ & 0 & $2 p$ & $q$ \\
\hline 28 & K14 & $\frac{1}{2} \mathbb{k}$ & $\frac{1}{2}$ & & $|s|^{2} H-\mathrm{B}(s \llbracket \bar{s})$ & - & - & - & - \\
\hline 29 & K15 & $\mathbb{k}$ & & & $-\mathrm{P}(s \mathbb{k} \bar{s})$ & - & - & - & - \\
\hline 30 & K15 & & & & $-\mathrm{P}(s \mathbb{k} \bar{s})$ & - & - & - & - \\
\hline 31 & K15 & & & & $|s|^{2} H$ & $2 q$ & $2 m$ & $4 m$ & $q$ \\
\hline 32 & K15 & & & & $|s|^{2} H-\mathrm{P}(s \mathbb{k} \bar{s})$ & - & - & - & - \\
\hline $33_{\lambda \in \mathbb{R}}$ & K16 & $\frac{1}{2}(1+\lambda \mathbb{k})$ & & & $-\mathrm{P}(s \mathbb{k} \bar{s})$ & 0 & 0 & $2 q$ & $q$ \\
\hline 34 & K17 & $\frac{1}{2} \mathbb{k}$ & & & $-\mathrm{P}(s \mathbb{k} \bar{s})$ & - & - & - & - \\
\hline $35_{\lambda \in \mathbb{R}}$ & K18 & $1+\lambda \mathbb{k}$ & & & $-\mathrm{P}(s \mathbb{k} \bar{s})$ & - & - & - & - \\
\hline
\end{tabular}

Table 4. Kinematical Lie superalgebras (with $[\boldsymbol{Q}, \boldsymbol{Q}] \neq 0$ ).

The first column is our identifier for $\mathfrak{s}$, whereas the second column is the kinematical Lie algebra $\mathfrak{k}=\mathfrak{s}_{\overline{0}}$ in table 2 . The next four columns specify the brackets of $\mathfrak{s}$ not of the form $[\boldsymbol{J},-]$. Supercharges $\mathrm{Q}(s)$ are parametrised by $s \in \mathbb{H}$, whereas $\mathrm{J}(\omega), \mathrm{B}(\beta)$ and $\mathrm{P}(\pi)$ are parametrised by $\omega, \beta, \pi \in \operatorname{Im} \mathbb{H}$. The brackets are given by $[H, \mathrm{Q}(s)]=\mathrm{Q}(s \mathfrak{h}),[\mathrm{B}(\beta), \mathrm{Q}(s)]=$ $\mathrm{Q}(\beta s \mathfrak{b})$ and $[\mathrm{P}(\pi), \mathrm{Q}(s)]=\mathrm{Q}(\pi s \mathbb{P})$, for some $\mathbb{h}, \mathbb{b}, \mathbb{P} \in \mathbb{H}$. (This formalism is explained in section 2.2.) The final four columns specify compatible gradings of $\mathfrak{s}$, with $m, n, p, q \in \mathbb{Z}$ and $q$ odd. 


\begin{tabular}{|l|rr|l|}
\hline A\# & \multicolumn{2}{|c|}{ Nonzero Lie brackets } & \multicolumn{1}{|c|}{ Spacetime } \\
\hline 1 & & static \\
2 & {$[H, \boldsymbol{P}]=\boldsymbol{P}$} & torsional static \\
$3_{+}$ & & {$[\boldsymbol{P}, \boldsymbol{P}]=\boldsymbol{J}$} & $\mathbb{R} \times S^{3}$ \\
$3_{-}$ & & {$[\boldsymbol{P}, \boldsymbol{P}]=-\boldsymbol{J}$} & $\mathbb{R} \times H^{3}$ \\
\hline
\end{tabular}

Table 5. Aristotelian Lie algebras and their spacetimes.

basis so that $\mathbb{C}_{3}=\mathbb{k}$ and $\mathbb{h}=\mathbb{k}$ if nonzero. This leaves two possible Lie superalgebras depending on whether or not $\mathbb{h}=0$ :

$$
[H, \mathrm{Q}(s)]=\left\{\begin{array}{l}
\mathrm{Q}(s \mathbb{k}) \\
0
\end{array} \quad \text { and } \quad[\mathrm{Q}(s), \mathrm{Q}(s)]=-\mathrm{P}(s \mathbb{k} \bar{s}) .\right.
$$

2. If $c_{0} \neq 0$, then $\mathbb{h}=0$ and $\mathbb{C}_{3}$ is free. We can set $c_{0}=1$ and, if nonzero, we can also set $\mathbb{C}_{3}=\mathbb{k}$. This gives two possible Lie superalgebras:

$$
[\mathrm{Q}(s), \mathrm{Q}(s)]=\left\{\begin{array}{l}
|s|^{2} H \\
|s|^{2} H-\mathrm{P}(s \mathbb{k} \bar{s}) .
\end{array}\right.
$$

\subsubsection{Lie superalgebras associated with aristotelian Lie algebra A2}

Let us now consider the aristotelian Lie algebra $\mathrm{A} 2$, with additional bracket $[H, \boldsymbol{P}]=\boldsymbol{P}$. Lemma 1 again says $\mathbb{p}=0$ and Lemma 2 again says that $\mathbb{C}_{1}=2 c_{0}$ h. The $[H, \boldsymbol{Q}, \boldsymbol{Q}]$ component of the Jacobi identity implies that $c_{0} \operatorname{Re} h=0$ (which, again, is redundant), $\mathbb{C}_{1} \overline{\mathbb{h}}+\mathfrak{h}_{\mathbb{C}_{1}}=0$ and $\mathbb{C}_{3} \overline{\mathfrak{h}}+\mathfrak{h}_{\mathbb{C}_{3}}=\mathbb{C}_{3}$, whereas the $[\boldsymbol{P}, \boldsymbol{Q}, \boldsymbol{Q}]$ component results in $\left[s \mathbb{C}_{1} \bar{s}, \pi\right]=$ $2 c_{0}|s|^{2} \pi$ for all $\pi \in \operatorname{Im} \mathbb{H}$ and $s \in \mathbb{H}$. This can only be the case if $c_{0}=0$ and hence $\mathbb{C}_{1}=0$, which then forces $\mathbb{C}_{3} \neq 0$. The equation $\mathbb{C}_{3} \overline{\mathfrak{h}}+\mathfrak{h}_{\mathbb{C}_{3}}=\mathbb{C}_{3}$ results in $\left[\operatorname{Im} \mathbb{h}, \mathbb{C}_{3}\right]=(1-2 \operatorname{Re} \mathfrak{h}) \mathbb{C}_{3}$, which implies $\operatorname{Re} \mathbb{h}=\frac{1}{2}$ and $\operatorname{Im} \mathbb{h}$ collinear with $\mathbb{C}_{3}$. We can change basis so that $\mathbb{C}_{3}=\mathbb{k}$ and we end up with a one-parameter family of Lie superalgebras with brackets

$$
[H, \mathrm{Q}(s)]=\mathrm{Q}\left(\frac{1}{2} s(1+\lambda \mathbb{k})\right), \quad[\mathrm{Q}(s), \mathrm{Q}(s)]=-\mathrm{P}(s \mathbb{k} \bar{s})
$$

for $\lambda \in \mathbb{R}$, in addition to $[H, \mathrm{P}(\pi)]=\mathrm{P}(\pi)$.

\subsubsection{Lie superalgebras associated with aristotelian Lie algebras $A 3_{ \pm}$}

Finally, we consider the aristotelian Lie algebras $\mathrm{A}_{ \pm}$with bracket $[\boldsymbol{P}, \boldsymbol{P}]= \pm \boldsymbol{J}$. Lemma 1 says that $[\mathfrak{h}, \mathbb{p}]=0$ and $\mathbb{p}^{2}= \pm \frac{1}{4}$, whereas Lemma 2 says that $c_{0} \mathfrak{h}=\frac{1}{2} \mathbb{C}_{1}+\mathbb{C}_{3} \mathfrak{p}$. The $[H, \boldsymbol{Q}, \boldsymbol{Q}]$ Jacobi says $c_{0} \operatorname{Re} \mathfrak{h}=0, \mathbb{C}_{1} \overline{\mathfrak{h}}+\mathfrak{h}_{1}=0$ and $\mathbb{C}_{3} \overline{\mathfrak{h}}+\mathfrak{h}_{\mathbb{C}_{3}}=0$, whereas the $[\boldsymbol{P}, \boldsymbol{Q}, \boldsymbol{Q}]$ Jacobi gives the following relations:

$$
c_{0} \operatorname{Re}(\bar{s} \pi s \mathrm{p})=0, \quad \pi s \mathbb{p}_{3} \bar{s}-s \mathbb{C}_{3} \overline{\mathrm{p} s} \pi=\frac{1}{2}\left[\pi, s \mathbb{C}_{1} \bar{s}\right] \quad \text { and } \pi s \mathbb{p}_{1} \bar{s}-s \mathbb{C}_{1} \overline{\mathrm{P} s} \pi= \pm \frac{1}{2}\left[\pi, s \mathbb{C}_{3} \bar{s}\right]
$$

We must distinguish two cases depending on the choice of signs. 
1. Let's take the + sign. Then $\mathbb{p}^{2}=\frac{1}{4} \in \mathbb{R}$. Without loss of generality we can take $\mathbb{p}=\frac{1}{2}$ by changing the sign of $\boldsymbol{P}$ if necessary. Then the $[\boldsymbol{P}, \boldsymbol{Q}, \boldsymbol{Q}]$ Jacobi equations say that $\mathbb{C}_{1}=\mathbb{C}_{3}$ and hence $c_{0} \mathfrak{h}=\mathbb{C}_{1}$. If $c_{0}=0$, then $\mathbb{C}_{1}=\mathbb{C}_{3}=0$, hence we take $c_{0} \neq 0$ and thus $\operatorname{Re} \mathbb{h}=0$. We can change basis so that $c_{0}=1$ and hence $\mathbb{h}=\mathbb{C}_{1}=\mathbb{C}_{3}$. If nonzero, we can take them all equal to $\mathbb{k}$. In summary, we have two possible aristotelian Lie superalgebras extending $\mathrm{A} 3_{+}$, with brackets $\left[\mathrm{P}(\pi), \mathrm{P}\left(\pi^{\prime}\right)\right]=\frac{1}{2} \mathrm{~J}\left(\left[\pi, \pi^{\prime}\right]\right)$ and in addition either

$$
[\mathrm{P}(\pi), \mathrm{Q}(s)]=\mathrm{Q}\left(\frac{1}{2} \pi s\right), \quad \text { and } \quad[\mathrm{Q}(s), \mathrm{Q}(s)]=|s|^{2} H
$$

or

$[H, \mathrm{Q}(s)]=\mathrm{Q}(s \mathbb{k}), \quad[\mathrm{P}(\pi), \mathrm{Q}(s)]=\mathrm{Q}\left(\frac{1}{2} \pi s\right)$ and $[\mathrm{Q}(s), \mathrm{Q}(s)]=|s|^{2} H-\mathrm{J}(s \mathbb{k} \bar{s})-\mathrm{P}(s \mathbb{k} \bar{s})$.

2. Let us now take the - sign. Here $p^{2}=-\frac{1}{4}$, so that $p \in \operatorname{Im} H($ and $p \neq 0$ ) and hence $\operatorname{Im} t h$ collinear with $p$. The $[H, \boldsymbol{Q}, \boldsymbol{Q}]$ Jacobi equations force th $=0$ and the $[\boldsymbol{P}, \boldsymbol{Q}, \boldsymbol{Q}]$ Jacobi equations force $c_{0}=0$ and $\mathbb{C}_{3} \mathbb{P}=-\frac{1}{2} \mathbb{C}_{1}$. This means that $\left(\mathbb{C}_{1}, 2 \mathfrak{p}, \mathbb{C}_{3}\right)$ is an oriented orthonormal frame for $\operatorname{Im} \mathbb{H}$ and hence we can rotate them so that $\left(\mathbb{C}_{1}, 2 \mathfrak{p}, \mathbb{C}_{3}\right)=(-\mathfrak{j}, \stackrel{0}{0}, \mathbb{k})$, for later uniformity. This results in the aristotelian Lie superalgebra extending $\mathrm{A}_{-}$- by the following brackets in addition to $\left[\mathrm{P}(\pi), \mathrm{P}\left(\pi^{\prime}\right)\right]=\frac{1}{2} \mathrm{~J}\left(\left[\pi, \pi^{\prime}\right]\right):$

$$
[\mathrm{P}(\pi), \mathrm{Q}(s)]=\mathrm{Q}\left(\frac{1}{2} \pi s^{\circ}\right) \quad \text { and } \quad[\mathrm{Q}(s), \mathrm{Q}(s)]=\mathrm{J}\left(s \bar{s}_{\bar{s}}\right)-\mathrm{P}(s \llbracket \bar{s}) .
$$

These results are summarised in table 6 below, together with the possible compatible $\mathbb{Z}$-gradings. This table also classifies the homogeneous aristotelian superspaces.

\subsection{Unpacking the quaternionic notation}

The quaternionic formalism we have employed in the classification of kinematical and aristotelian Lie superalgebras, which has the virtue of uniformity and ease in computation, does result in expressions which are perhaps unfamiliar and which therefore might hinder comparison with other formulations. In this section, we will go through an example illustrating how to unpack the notation.

The nonzero brackets of the Poincaré superalgebra S14 are given by equation (2.5) and

$$
[\mathrm{B}(\beta), \mathrm{Q}(s)]=\mathrm{Q}\left(\frac{1}{2} \beta s \mathbb{k}\right) \quad \text { and } \quad[\mathrm{Q}(s), \mathrm{Q}(s)]=|s|^{2} H-\mathrm{P}(s \mathbb{k} \bar{s})
$$

where

$$
\mathrm{B}(\beta)=\sum_{i=1}^{3} \beta_{i} B_{i} \quad \text { and } \quad \mathrm{Q}(s)=\sum_{a=1}^{4} s_{a} Q_{a}
$$

and where

$$
\beta=\beta_{1} \AA+\beta_{2} \AA+\beta_{3} \mathbb{k} \quad \text { and } \quad s=s_{1} \AA+s_{2} \AA+s_{3} \mathbb{k}+s_{4} .
$$

This allows us to simply unpack the brackets into the following

$$
\left[B_{i}, Q_{a}\right]=\frac{1}{2} \sum_{b=1}^{4} Q_{b} \beta_{i}{ }^{b}{ }_{a} \quad \text { and } \quad\left[Q_{a}, Q_{b}\right]=\sum_{\mu=0}^{3} P_{\mu} \gamma_{a b}^{\mu}
$$




\begin{tabular}{|c|c|c|c|c|c|c|c|}
\hline $\mathrm{S} \#$ & $\mathfrak{a}$ & Th & $\mathrm{p}$ & {$[\mathrm{Q}(s), \mathrm{Q}(s)]$} & $w_{H}$ & $w_{\boldsymbol{P}}$ & $w_{Q}$ \\
\hline 36 & $\mathrm{~A} 1$ & $\mathbb{k}$ & & $-\mathrm{P}(s \mathbb{k} \bar{s})$ & 0 & $2 q$ & $q$ \\
\hline 37 & $\mathrm{~A} 1$ & & & $-\mathrm{P}(s \mathbb{k} \bar{s})$ & $2 n$ & $2 q$ & $q$ \\
\hline 38 & $\mathrm{~A} 1$ & & & $|s|^{2} H$ & $2 q$ & $2 p$ & $q$ \\
\hline 39 & $\mathrm{~A} 1$ & & & $|s|^{2} H-\mathrm{P}(s \llbracket \bar{s})$ & $2 q$ & $2 q$ & $q$ \\
\hline $40_{\lambda \in \mathbb{R}}$ & $\mathrm{A} 2$ & $\frac{1}{2}(1+\lambda \mathbb{k})$ & & $-\mathrm{P}(s \mathbb{k} \bar{s})$ & 0 & $2 q$ & $q$ \\
\hline 41 & $\mathrm{~A} 3_{+}$ & & $\frac{1}{2}$ & $|s|^{2} H$ & $2 q$ & 0 & $q$ \\
\hline 42 & $\mathrm{~A} 3_{+}$ & $\mathbb{k}$ & $\frac{1}{2}$ & $|s|^{2} H-\mathrm{J}(s \mathbb{k} \bar{s})-\mathrm{P}(s \mathbb{k} \bar{s})$ & - & - & - \\
\hline 43 & $\mathrm{~A} 3_{-}$ & & $\frac{1}{2} \AA$ & $\mathrm{J}\left(s \rho_{\bar{s}}\right)-\mathrm{P}(s \llbracket \bar{s})$ & - & - & - \\
\hline
\end{tabular}

Table 6. Aristotelian Lie superalgebras (with $[Q, Q] \neq 0$ ).

The first column is our identifier for $\mathfrak{s}$, whereas the second column is the aristotelian Lie algebra $\mathfrak{a}=\mathfrak{s}_{\overline{0}}$ in table 5 . The next three columns specify the brackets of $\mathfrak{s}$ not of the form $[\boldsymbol{J},-]$. Supercharges $\mathrm{Q}(s)$ are parametrised by $s \in \mathbb{H}$, whereas $\mathrm{J}(\omega)$ and $\mathrm{P}(\pi)$ are parametrised by $\omega, \pi \in \operatorname{Im} H$. The brackets are given by $[H, \mathrm{Q}(s)]=\mathrm{Q}(s h)$ and $[\mathrm{P}(\pi), \mathrm{Q}(s)]=$ $\mathrm{Q}(\pi s \mathrm{p})$, for some $\mathfrak{h}, \mathbb{p} \in \mathbb{H}$. (The formalism is explained in section 2.2.) The final three columns are compatible gradings of $\mathfrak{s}$, with $n, p, q \in \mathbb{Z}$ and $q$ odd.

where we have introduced $P_{0}=H$ and where the matrices $\boldsymbol{\beta}_{i}:=\left[\beta_{i}{ }_{a}{ }\right]$ are given by

$$
\boldsymbol{\beta}_{1}=\left(\begin{array}{cc}
0 & -\mathbb{1} \\
-\mathbb{1} & 0
\end{array}\right), \quad \boldsymbol{\beta}_{2}=\left(\begin{array}{cc}
0 & i \sigma_{2} \\
-i \sigma_{2} & 0
\end{array}\right) \quad \text { and } \quad \boldsymbol{\beta}_{3}=\left(\begin{array}{cc}
\mathbb{1} & 0 \\
0 & -\mathbb{1}
\end{array}\right)
$$

and where the symmetric matrices $\gamma^{\mu}:=\left[\gamma_{a b}^{\mu}\right]$ are given by

$$
\boldsymbol{\gamma}^{0}=\left(\begin{array}{ll}
\mathbb{1} & 0 \\
0 & \mathbb{1}
\end{array}\right), \quad \boldsymbol{\gamma}^{1}=\left(\begin{array}{ll}
0 & \mathbb{1} \\
\mathbb{1} & 0
\end{array}\right), \quad \boldsymbol{\gamma}^{2}=\left(\begin{array}{cc}
0 & -i \sigma_{2} \\
i \sigma_{2} & 0
\end{array}\right) \quad \text { and } \quad \boldsymbol{\gamma}^{3}=\left(\begin{array}{cc}
-\mathbb{1} & 0 \\
0 & \mathbb{1}
\end{array}\right) \text {. }
$$

As shown in section 4.6, there is a two-parameter family of symplectic forms on the spinor representation $S$ which are invariant under the action of $B_{i}$ and $J_{i}$. They are given by

$$
\omega\left(s_{1}, s_{2}\right):=\operatorname{Re}\left(s_{1}\left(\alpha \stackrel{\circ}{0} \beta_{j}\right) \bar{s}_{2}\right),
$$

for $\alpha, \beta \in \mathbb{R}$ not both zero. We may normalise $\omega$ such that $\alpha^{2}+\beta^{2}=1$, resulting in a circle of symplectic structures. Relative to the standard real basis $(\stackrel{0}{0}, j, \mathbb{k}, 1)$ for $\mathbb{H}$, the matrix $\Omega$ of $\omega$ is given by $\Omega=i \sigma_{2} \otimes\left(-\alpha \sigma_{1}+\beta \sigma_{3}\right)$, whose inverse is $\Omega^{-1}=-\Omega$, due to the chosen normalisation. Let us define endomorphisms $\gamma^{\mu}$ of $S$ such that $\left(\gamma^{\mu}\right)^{a}{ }_{b}=\left(\Omega^{-1}\right)^{a c} \gamma_{c b}^{\mu}$. Explicitly, they are given by

$$
\begin{array}{ll}
\gamma^{0}=i \sigma_{2} \otimes\left(\alpha \sigma_{1}-\beta \sigma_{3}\right) & \gamma^{2}=-\mathbb{1} \otimes\left(\alpha \sigma_{3}+\beta \sigma_{1}\right) \\
\gamma^{1}=\sigma_{3} \otimes\left(\alpha \sigma_{1}-\beta \sigma_{3}\right) & \gamma^{3}=\sigma_{1} \otimes\left(\alpha \sigma_{1}-\beta \sigma_{3}\right) .
\end{array}
$$

It then follows that these endomorphisms represent the Clifford algebra $C \ell(1,3)$ :

$$
\gamma^{\mu} \gamma^{\nu}+\gamma^{\nu} \gamma^{\mu}=-2 \eta^{\mu \nu} \mathbb{1}
$$

We thus arrive at the description of the Poincaré superalgebra described in the appendix. 


\subsection{Central extensions}

In this section, we determine the possible central extensions of the kinematical and aristotelian Lie superalgebras.

We start with the kinematical Lie superalgebras. Let $\mathfrak{s}=\mathfrak{s}_{\overline{0}} \oplus \mathfrak{s}_{\overline{1}}$ be one of the Lie superalgebras in table 4 . By a central extension of $\mathfrak{s}$, we mean a short exact sequence of Lie superalgebras

$$
0 \longrightarrow \mathfrak{z} \longrightarrow \mathfrak{\mathfrak { s }} \longrightarrow \mathfrak{s} \longrightarrow 0,
$$

where $\mathfrak{z}$ is central in $\widehat{\mathfrak{s}}$. We may choose a vector space splitting and view (as a vector space) $\widehat{\mathfrak{s}}=\mathfrak{s} \oplus \mathfrak{z}$ and the Lie bracket is given, for $(X, z),\left(Y, z^{\prime}\right) \in \mathfrak{s} \oplus \mathfrak{z}$, by

$$
\left[(X, z),\left(Y, z^{\prime}\right)\right]_{\mathfrak{s}}=\left([X, Y]_{\mathfrak{s}}, \omega(X, Y)\right)
$$

where $\omega: \wedge^{2} \mathfrak{s} \rightarrow \mathfrak{z}$ is a cocycle. (Here $\wedge$ is taken in the super sense, so that it is symmetric on odd elements.) Central extensions of $\mathfrak{s}$ are classified up to isomorphism by the Chevalley-Eilenberg cohomology group $H^{2}(\mathfrak{s})$, which by Hochschild-Serre, can be computed from the subcomplex relative to the rotational subalgebra $\mathfrak{r} \subset \mathfrak{s}_{\overline{0}}$. Indeed, we have the isomorphism [17]

$$
H^{2}(\mathfrak{s}) \cong H^{2}(s, \mathfrak{r}) .
$$

Let $W=\operatorname{span}_{\mathbb{R}}\{H, \boldsymbol{B}, \boldsymbol{P}, \boldsymbol{Q}\}$. Then the cochains in $C^{2}(s, \mathfrak{r})$ are $\mathfrak{r}$-equivariant maps $\wedge^{2} W \rightarrow$ $\mathbb{R}$ or, equivalently, $\mathfrak{r}$-invariant vectors in $\wedge^{2} W^{*}$. This is a two-dimensional real vector space which, in quaternionic language, is given for $x, y \in \mathbb{R}$ by

$$
\omega(\mathrm{B}(\beta), \mathrm{P}(\pi))=x \operatorname{Re}(\beta \pi)=-\omega(\mathrm{P}(\pi), \mathrm{B}(\beta)) \quad \text { and } \quad \omega\left(\mathrm{Q}\left(s_{1}\right), \mathrm{Q}\left(s_{2}\right)\right)=y \operatorname{Re}\left(s_{1} \bar{s}_{2}\right) .
$$

The cocycle conditions (i.e., the Jacobi identities of the central extension $\widehat{\mathfrak{s}}$ ) has several components. Letting $\mathrm{V}$ stand for either $\mathrm{B}$ or $\mathrm{P}$, the cocycle conditions are given by

$$
\begin{array}{r}
\omega([H, \mathrm{~V}(\alpha)], \mathrm{V}(\beta))+\omega(\mathrm{V}(\alpha),[H, \mathrm{~V}(\beta)])=0, \\
\omega([\mathrm{V}(\alpha), \mathrm{V}(\beta)], \mathrm{V}(\gamma))+\text { cyclic }=0, \\
\omega([H, \mathrm{Q}(s)], \mathrm{Q}(s))=0, \\
2 \omega([\mathrm{V}(\alpha), \mathrm{Q}(s)], \mathrm{Q}(s))+\omega([\mathrm{Q}(s), \mathrm{Q}(s)], \mathrm{V}(\alpha))=0 .
\end{array}
$$

The first two of the above equations only involve the even generators and hence depend only on the underlying kinematical Lie algebra, whereas the last two equations do depend on the precise superalgebra we are dealing with. In the case of aristotelian Lie superalgebras, there is no $\boldsymbol{B}$ and hence $\mathrm{V}=\mathrm{P}$ in the above equations and, of course, the cocycle can only modify the $[\boldsymbol{Q}, \boldsymbol{Q}]$ bracket and hence the cocycle conditions are simply

$$
\omega([H, \mathrm{Q}(s)], \mathrm{Q}(s))=0 \quad \text { and } \quad \omega([\mathrm{P}(\alpha), \mathrm{Q}(s)], \mathrm{Q}(s))=0 .
$$

The calculations are routine, and we will not give any details, but simply collect the results in table 7 , where $Z$ is the basis for the one-dimensional central ideal $\mathfrak{z}=\operatorname{span}_{\mathbb{R}}\{Z\}$, and where we list only the brackets which are liable to change under central extension. 


\begin{tabular}{|c|c|c|}
\hline $\mathrm{S} \#$ & {$[\mathrm{~B}(\beta), \mathrm{P}(\pi)]$} & {$[\mathrm{Q}(s), \mathrm{Q}(s)]$} \\
\hline 1 & & $|s|^{2} Z-\mathrm{P}(s \mathbb{k} \bar{s})$ \\
\hline 4 & $-\operatorname{Re}(\beta \pi) Z$ & $|s|^{2} H$ \\
\hline 5 & & 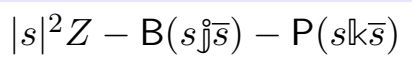 \\
\hline 6 & & $|s|^{2} Z-\mathrm{P}(s \mathbb{k} \bar{s})$ \\
\hline 7 & & $|s|^{2} Z-\mathrm{P}(s \mathbb{k} \bar{s})$ \\
\hline 8 & & $|s|^{2} Z-\mathrm{P}(s \mathbb{k} \bar{s})$ \\
\hline $10_{\gamma=0, \lambda \in \mathbb{R}}$ & & $|s|^{2} Z-\mathrm{B}(s \mathbb{k} \bar{s})$ \\
\hline $11_{\chi=0}$ & & $|s|^{2} Z-\mathrm{B}\left(s \varsigma^{\circ} \bar{s}\right)-\mathrm{P}(s \llbracket \bar{s})$ \\
\hline 13 & $-\operatorname{Re}(\beta \pi)(H+Z)$ & $|s|^{2} H$ \\
\hline 23 & & $|s|^{2} Z-\mathrm{P}(s \mathbb{k} \bar{s})$ \\
\hline 24 & & $|s|^{2} Z-\mathrm{P}(s \llbracket \bar{s})$ \\
\hline 29 & & $|s|^{2} Z-\mathrm{P}(s \mathbb{k} \bar{s})$ \\
\hline 30 & & $|s|^{2} Z-\mathrm{P}(s \mathbb{k} \bar{s})$ \\
\hline 34 & & $|s|^{2} Z-\mathrm{P}(s \mathbb{k} \bar{s})$ \\
\hline 36 & - & $|s|^{2} Z-\mathrm{P}(s \mathbb{k} \bar{s})$ \\
\hline 37 & - & $|s|^{2} Z-\mathrm{P}(s \mathbb{k} \bar{s})$ \\
\hline
\end{tabular}

Table 7. Central extensions of kinematical and aristotelian Lie superalgebras.

The first column is our identifier for $\mathfrak{s}$, whereas the other two columns are the possible central terms in the central extension $\widehat{\mathfrak{s}}$. Here $\beta, \pi \in \operatorname{Im} \mathbb{H}$ and $s \in \mathbb{H}$ are (some of) the parameters defining the Lie brackets in the quaternionic formalism explained in section 2.2.

\subsection{Automorphisms of kinematical Lie superalgebras}

In the next section, we will classify the homogeneous superspaces associated to the kinematical Lie superalgebras. As we will explain below, the first stage is to classify "super Lie pairs" up to isomorphism. To that end, it behoves us to determine the group of automorphisms of the Lie superalgebras in table 4, to which we now turn.

Without loss of generality, we can restrict to automorphisms which are the identity when restricted to $\mathfrak{r}$ : we call them $\mathfrak{r}$-fixing automorphisms. Following from our discussion in section 2.5 , these are parametrised by triples

$$
\left(A:=\left(\begin{array}{ll}
a & b \\
c & d
\end{array}\right), \mu, q\right) \in \operatorname{GL}(2, \mathbb{R}) \times \mathbb{R}^{\times} \times \mathbb{H}^{\times}
$$

subject to the condition that the associated linear transformations leave the Lie brackets in $\mathfrak{s}$ unchanged.

It is easy to read off from equation (2.39) what $(A, \mu, \mathbb{q})$ must satisfy for the $\mathfrak{r}$ equivariant linear transformation $\Phi: \mathfrak{s} \rightarrow \mathfrak{s}$ defined by them to be an automorphism 
of $\mathfrak{s}$, namely:

$$
\begin{aligned}
& \text { Thq }=\mu q \mathbb{h} \\
& \mathfrak{b q}=\mathbb{q}(a \mathfrak{b}+c \mathfrak{p}) \\
& \mathrm{pq}=\mathbb{q}(b \mathfrak{b}+d \mathfrak{p}) \\
& \mu c_{0}=|\mathbb{q}|^{2} c_{0} \\
& \mathbb{Q}_{1} \overline{\mathbb{q}}=\mathbb{C}_{1} \\
& \mathbb{q \mathbb { C }}_{2} \overline{\mathbb{q}}=a \mathbb{C}_{2}+b \mathbb{C}_{3} \\
& \mathbb{q \mathbb { C }}_{3} \overline{\mathbb{q}}=c \mathbb{C}_{2}+d \mathbb{\mathbb { C }}_{3} .
\end{aligned}
$$

It is then a straightforward - albeit lengthy — process to go through each Lie superalgebra in table 4 and solve equations (3.75) for $(A, \mu, \mathfrak{q})$. In particular, $(A, \mu) \in$ Aut $_{\mathfrak{r}}(\mathfrak{k})$ and they are given in table 3 . The results of this section are summarised in tables 8 and 9 , which list the $\mathfrak{r}$-fixing automorphisms for the Lie superalgebras S1-S15 and S16-S35, respectively, in table 4 .

The first six Lie superalgebras in table 4 are supersymmetric extensions of the static kinematical Lie algebra for which $(A, \mu)$ can be any element in $\operatorname{GL}(2, \mathbb{R}) \times \mathbb{R}^{\times}$.

\subsubsection{Automorphisms of Lie superalgebra S1}

Here $\mathbb{h}=\frac{1}{2} \mathbb{k}, \mathbb{b}=\mathbb{p}=0, c_{0}=0, \mathbb{C}_{1}=\mathbb{C}_{2}=0$ and $\mathbb{C}_{3}=\mathbb{k}$. The invariance conditions (3.75) give

$$
\mu q \mathbb{k}=\mathbb{k} q, \quad b \mathbb{k}=0 \quad \text { and } \quad d \mathbb{k}=\mathbb{q} k \bar{q}
$$

The second equation requires $b=0$. The third equation says that the real linear map $\alpha_{\mathbb{q}}: \mathbb{H} \rightarrow \mathbb{H}$ defined by $\alpha_{\mathbb{q}}(\mathfrak{x})=\mathbb{q} \times \overline{\mathbb{q}}$ preserves the $\mathbb{k}$-axis in $\operatorname{Im} \mathbb{H}$.

Lemma 3. Let $\mathbb{q} \mathbb{k} \bar{q}=d q$ for some $d \in \mathbb{R}$. Then either $d=|q|^{2}$ and $\mathbb{q} \in \operatorname{span}_{\mathbb{R}}\{1, \mathbb{k}\}$ or $d=-|\mathbb{q}|^{2}$ and $\mathbb{q} \in \operatorname{span}_{\mathbb{R}}\{\stackrel{0}{0}, \mathfrak{j}\}$.

Proof. Taking the quaternion norm of both sides of the equation $\mathbb{q} \bar{k} \bar{q}=d q$ and using that $\mathbb{q} \neq 0$, we see that $d= \pm|\mathbb{q}|^{2}$ and hence right multiplying by $\mathbb{q}$, the equation becomes $\pm \mathbb{k} \mathfrak{q}=\mathbb{q} \mathbb{k}$. If $\mathbb{k} \mathbb{q}=\mathbb{q} \mathbb{k}$, then $\mathbb{q} \in \operatorname{span}_{\mathbb{R}}\{1, \mathbb{k}\}$ and $d=|\mathfrak{q}|^{2}$, whereas if $-\mathbb{k} \mathbb{q}=\mathbb{q} \mathbb{k}$, then $\mathbb{q} \in \operatorname{span}_{\mathbb{R}}\{\stackrel{\circ}{0}, \stackrel{\jmath}{\jmath}\}$ and $d=-|q|^{2}$.

Taking the quaternion norm of the first equation, shows that $\mu= \pm 1$ and hence that $d=\mu|\mathbb{q}|^{2}$. In summary, we have that the typical automorphism $(A, \mu, \mathbb{q})$ takes one of two possible forms:

$$
\begin{aligned}
A & =\left(\begin{array}{cc}
a & 0 \\
c & |\mathbb{q}|^{2}
\end{array}\right), \quad \mu=1 \quad \text { and } \quad \mathbb{q}=q_{4}+q_{3} \mathbb{k} \\
\text { or } \quad A & =\left(\begin{array}{cc}
a & 0 \\
c & -|\mathfrak{q}|^{2}
\end{array}\right), \quad \mu=-1 \quad \text { and } \quad \mathbb{q}=q_{1} \AA+q_{2} \AA .
\end{aligned}
$$

\subsubsection{Automorphisms of Lie superalgebra S2}

Here $\mathbb{h}=\mathbb{b}=\mathfrak{p}=0, c_{0}=1, \mathbb{C}_{1}=0, \mathbb{C}_{2}=\mathfrak{\jmath}$ and $\mathbb{C}_{3}=\mathbb{k}$. The invariance conditions (3.75) give

$$
\mu=|\mathbb{q}|^{2}, \quad a ̊+b \mathbb{k}=\mathbb{q} \AA \bar{\Phi} \quad \text { and } \quad c \AA+d \mathbb{k}=q \mathbb{q} k \overline{\mathbb{q}} .
$$

The last two equations say that the real linear map $\alpha_{\llbracket}: \mathbb{H} \rightarrow \mathbb{H}$ defined earlier preserves

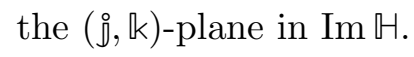


Lemma 4. The map $\alpha_{\mathbb{q}}: \mathbb{H} \rightarrow \mathbb{H}$ preserves the $(\stackrel{\wp}{\mathbb{j}}, \mathbb{k})$-plane in $\operatorname{Im} \mathbb{H}$ if and only if $\mathbb{q} \in$ $\operatorname{span}_{\mathbb{R}}\{1, \stackrel{\circ}{0}\} \cup \operatorname{span}_{\mathbb{R}}\{\mathfrak{j}, \mathbb{k}\}$.

Proof. Since $q \neq 0$, we can write it as $q=|q| u$, for some unique $u \in \operatorname{Sp}(1)$ and $\alpha_{\mathbb{q}}=|\mathbb{q}|^{2} \alpha_{u}$. The map $\alpha_{\mathbb{q}}$ preserves separately the real and imaginary subspaces of $\mathbb{H}$ and $\alpha_{\mathbb{q}}$ preserves

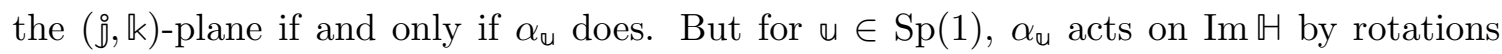

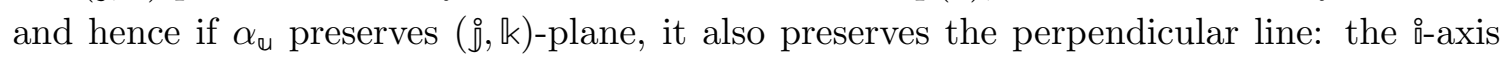

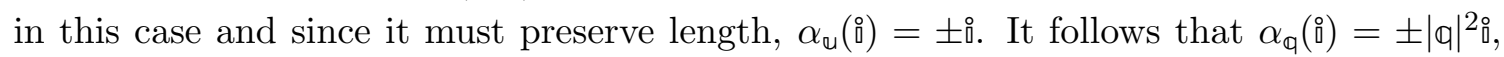
so that $\alpha_{\mathbb{q}}$ too preserves the $\stackrel{\AA}{\mathrm{d}}$-axis. By an argument similar to that of Lemma 3 it follows that $q$ belongs either to the complex line in $\mathbb{H}$ generated by $₫$ or to its perpendicular complement.

From the Lemma we have two cases to consider: $\mathbb{q}=q_{4}+q_{1} \stackrel{\circ}{\text { or }} \mathfrak{q}=q_{2} \AA+q_{3} \mathbb{k}$. In each case we can use the last two equations to solve for $a, b, c, d$ in terms of the components of $q$. Summarising, we have that the typical automorphism $(A, \mu, \mathbb{q})$ takes one of two possible forms:

$$
\begin{aligned}
A & =\left(\begin{array}{cc}
q_{4}^{2}-q_{1}^{2} & 2 q_{1} q_{4} \\
-2 q_{1} q_{4} & q_{4}^{2}-q_{1}^{2}
\end{array}\right), \quad \mu=q_{1}^{2}+q_{4}^{2} \quad \text { and } \quad q=q_{4}+q_{1} \AA \\
\text { or } \quad A & =\left(\begin{array}{cc}
q_{2}^{2}-q_{3}^{2} & 2 q_{2} q_{3} \\
2 q_{2} q_{3} & q_{3}^{2}-q_{2}^{2}
\end{array}\right), \quad \mu=q_{2}^{2}+q_{3}^{2} \quad \text { and } \quad \mathbb{q}=q_{2} \AA+q_{3} \mathbb{k} .
\end{aligned}
$$

\subsubsection{Automorphisms of Lie superalgebra S3}

Here $\mathbb{h}=\mathbb{b}=\mathfrak{p}=0, c_{0}=1, \mathbb{C}_{1}=\mathbb{C}_{2}=0$ and $\mathbb{C}_{3}=\mathbb{k}$. The invariance conditions (3.75) give

$$
\mu=|\mathbb{q}|^{2}, \quad b \mathbb{k}=0 \quad \text { and } \quad d \mathbb{k}=\mathbb{q} \mathbb{k} \overline{\mathfrak{q}} .
$$

This is very similar to the case of the Lie superalgebra S1 and, in particular, Lemma 3 applies. The typical automorphism $(A, \mu, \mathbb{q})$ takes one of two possible forms:

$$
\begin{aligned}
A & =\left(\begin{array}{cc}
a & 0 \\
c & |\mathfrak{q}|^{2}
\end{array}\right), \quad \mu=|\mathfrak{q}|^{2} \quad \text { and } \quad \mathbb{q}=q_{4}+q_{3} \mathbb{k} \\
\text { or } \quad A & =\left(\begin{array}{cc}
a & 0 \\
c & -|\mathbb{q}|^{2}
\end{array}\right), \quad \mu=|\mathbb{q}|^{2} \quad \text { and } \quad \mathbb{q}=q_{1} \AA+q_{2} \AA .
\end{aligned}
$$

\subsubsection{Automorphisms of Lie superalgebra S4}

Here $\mathfrak{h}=\mathbb{b}=\mathfrak{p}=0, c_{0}=1$ and $\mathbb{C}_{1}=\mathbb{C}_{2}=\mathbb{C}_{3}=0$. The only condition is $\mu=|\mathfrak{q}|^{2}$. Hence the typical automorphism $(A, \mu, \mathbb{q})$ takes the form

$$
A=\left(\begin{array}{ll}
a & b \\
c & d
\end{array}\right), \quad \mu=|\mathbb{q}|^{2} \quad \text { and } \quad \mathbb{q} \in \mathbb{H}^{\times} .
$$




\subsubsection{Automorphisms of Lie superalgebra S5}

Here $\mathbb{h}=\mathbb{b}=\mathfrak{p}=0, c_{0}=0, \mathbb{C}_{1}=0, \mathbb{C}_{2}=£$ and $\mathbb{C}_{3}=\mathbb{k}$. The invariance conditions (3.75) are as for Lie superalgebra $\mathrm{S} 2$, except that $\mu$ is unconstrained. In other words, the typical automorphism $(A, \mu, \mathbb{q})$ takes one of two possible forms:

$$
\begin{aligned}
A & =\left(\begin{array}{cc}
q_{4}^{2}-q_{1}^{2} & 2 q_{1} q_{4} \\
-2 q_{1} q_{4} & q_{4}^{2}-q_{1}^{2}
\end{array}\right), \quad \mu \quad \text { and } \quad \mathbb{q}=q_{4}+q_{1} \AA \\
\text { or } \quad A & =\left(\begin{array}{cc}
q_{2}^{2}-q_{3}^{2} & 2 q_{2} q_{3} \\
2 q_{2} q_{3} & q_{3}^{2}-q_{2}^{2}
\end{array}\right), \quad \mu \quad \text { and } \quad \mathbb{q}=q_{2} \AA+q_{3} \mathbb{k} .
\end{aligned}
$$

\subsubsection{Automorphisms of Lie superalgebra S6}

Here $\mathbb{h}=\mathbb{b}=\mathfrak{p}=0, c_{0}=0, \mathbb{C}_{1}=\mathbb{C}_{2}=0$ and $\mathbb{C}_{3}=\mathbb{k}$. This is similar to Lie superalgebra $\mathrm{S} 3$, except that $\mu$ remains unconstrained. In summary, the typical automorphisms $(A, \mu, \mathbb{q})$ takes one of two possible forms:

$$
\begin{aligned}
& A=\left(\begin{array}{cc}
a & 0 \\
c & |\mathfrak{q}|^{2}
\end{array}\right), \quad \mu \quad \text { and } \quad \mathbb{q}=q_{4}+q_{3} \mathbb{k}
\end{aligned}
$$

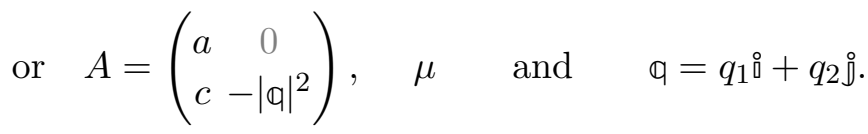

The next two Lie superalgebras (S7 and S8) are supersymmetric extensions of the galilean Lie algebra, where $(A, \mu)$ take the form

$$
A=\left(\begin{array}{ll}
a & b \\
c & d
\end{array}\right) \quad \text { and } \quad \mu=\frac{d}{a} .
$$

\subsubsection{Automorphisms of Lie superalgebra S7}

Here $\mathbb{h}=\mathbb{k}, \mathfrak{b}=\mathfrak{p}=0, c_{0}=0, \mathbb{C}_{1}=\mathbb{C}_{2}=0$ and $\mathbb{C}_{3}=\mathbb{k}$. The invariance conditions (3.75) are

$$
d \mathbb{q} \mathbb{k}=a \mathbb{k} \mathfrak{q} \quad \text { and } \quad d \mathbb{k}=\mathbb{q} \mathbb{k} \bar{q} .
$$

Multiplying the second equation on the right by $\mathbb{q}$, using the first equation and the fact that $q \neq 0$, results in $a=d^{2} /|q|^{2}$, so that $a>0$. Taking the quaternion norm of the first equation shows that $a=|d|$, so that $a=|\mathbb{q}|^{2}$. The first equation now follows from the second, and that is solved by Lemma 3 .

In summary the typical automorphism $(A, \mu, \mathbb{q})$ takes one of two possible forms:

$$
\begin{array}{rlrl}
A & =\left(\begin{array}{cc}
|\mathbb{q}|^{2} & 0 \\
c & |\mathbb{q}|^{2}
\end{array}\right), \quad \mu=1 \quad \text { and } & \mathbb{q}=q_{4}+q_{3} \mathbb{k} \\
\text { or } \quad A & =\left(\begin{array}{cc}
|\mathbb{q}|^{2} & 0 \\
c & -|\mathbb{q}|^{2}
\end{array}\right), \quad \mu=-1 \quad \text { and } \quad \mathbb{q}=q_{1} \stackrel{\mathbb{1}}{ }+q_{2} \AA .
\end{array}
$$




\subsubsection{Automorphisms of Lie superalgebra S8}

Here $\mathbb{h}=\mathbb{b}=\mathbb{p}=0, c_{0}=0, \mathbb{C}_{1}=\mathbb{C}_{2}=0$ and $\mathbb{C}_{3}=\mathbb{k}$. The invariance conditions (3.75) reduce to just $d \mathbb{k}=q \mathbb{k} \overline{\mathfrak{q}}$, which we solve by Lemma 3 . In summary, the typical automorphism $(A, \mu, \mathfrak{q})$ is as in the previous Lie superalgebra, except that $a$ is unconstrained (but nonzero). It can thus take one of two possible forms:

$$
\begin{array}{rlrl}
A & =\left(\begin{array}{cc}
a & 0 \\
c & |\mathfrak{q}|^{2}
\end{array}\right), \quad \mu=\frac{|\mathfrak{q}|^{2}}{a} & \text { and } & \mathbb{q}=q_{4}+q_{3} \mathbb{k} \\
\text { or } \quad A & =\left(\begin{array}{cc}
a & 0 \\
c & -|\mathbb{q}|^{2}
\end{array}\right), \quad \mu=-\frac{|\mathfrak{q}|^{2}}{a} \quad \text { and } \quad \mathbb{q}=q_{1} \AA+q_{2} \supseteqq .
\end{array}
$$

The next two classes of Lie superalgebras are associated with the one-parameter family of kinematical Lie algebras $\mathrm{K}_{\gamma}$, whose typical automorphisms $(A, \mu)$ depend on the value of $\gamma \in[-1,1]$. In the interior of the interval, it takes the form

$$
A=\left(\begin{array}{ll}
a & 0 \\
0 & d
\end{array}\right) \quad \text { and } \quad \mu=1
$$

but at the boundaries this is enhanced: at $\gamma=-1$ one can also have automorphisms of the form

$$
A=\left(\begin{array}{ll}
0 & b \\
c & 0
\end{array}\right) \quad \text { and } \quad \mu=-1,
$$

whereas at $\gamma=1$, the typical automorphism takes the form

$$
A=\left(\begin{array}{ll}
a & b \\
c & d
\end{array}\right) \quad \text { and } \quad \mu=1 .
$$

\subsubsection{Automorphisms of Lie superalgebra $S 9_{\gamma, \lambda}$}

Here $\mathbb{h}=\frac{1}{2}(1+\lambda \mathbb{k}), \mathbb{b}=\mathbb{p}=0, c_{0}=0, \mathbb{C}_{1}=\mathbb{C}_{2}=0$ and $\mathbb{C}_{3}=\mathbb{k}$. The invariance conditions (3.75) reduce to $b=0$ and, in addition,

$$
\mu \mathbb{q}(1+\lambda \mathbb{k})=(1+\lambda \mathbb{k}) \mathbb{q} \quad \text { and } \quad d \mathbb{k}=\mathbb{q} \mathbb{k} \overline{\mathbb{q}} .
$$

Taking the norm of the first equation, we find that $\mu= \pm 1$. If $\mu=1$, then $\lambda[\mathbb{k}, \mathbb{q}]=0$ so that either $\lambda \neq 0$, in which case $\mathbb{q} \in \operatorname{span}_{\mathbb{R}}\{1, \mathbb{k}\}$ or $\lambda=0$ and $\mathbb{q}$ is not constrained by this equation. The second equation is dealt with by Lemma 3 , which implies in particular that $d= \pm\left.|q|\right|^{2}$ and since $q \neq 0, d \neq 0$. This precludes the case $\mu=-1$ by inspecting the possible automorphisms $(A, \mu)$ of $\mathfrak{k}$. In summary, for generic $\gamma$ and $\lambda$, the typical automorphism $(A, \mu, \mathbb{q})$ takes the form

$$
A=\left(\begin{array}{cc}
a & 0 \\
0 & |q|^{2}
\end{array}\right), \quad \mu=1 \quad \text { and } \quad \mathbb{q}=q_{4}+q_{3} \mathbb{k},
$$

which is enhanced for $\gamma=1$ (but $\lambda$ still generic) to

$$
A=\left(\begin{array}{cc}
a & 0 \\
c & |\mathbb{q}|^{2}
\end{array}\right), \quad \mu=1 \quad \text { and } \quad \mathbb{q}=q_{4}+q_{3} \mathbb{k} .
$$


If $\lambda=0$, then the automorphisms are enhanced by the addition of $(A, \mu, \mathbb{q})$ of the form

$$
A=\left(\begin{array}{cc}
a & 0 \\
0 & -|q|^{2}
\end{array}\right), \quad \mu=1 \quad \text { and } \quad \mathbb{q}=q_{1} \AA+q_{2} \AA
$$

for generic $\gamma$ or, for $\gamma=1$ only, also

$$
A=\left(\begin{array}{cc}
a & 0 \\
c & -|q|^{2}
\end{array}\right), \quad \mu=1 \quad \text { and } \quad \mathbb{q}=q_{1} \stackrel{\imath}{ }+q_{2} \AA .
$$

\subsubsection{Automorphisms of Lie superalgebra $\mathrm{S} 10_{\gamma, \lambda}$}

Here $\mathbb{h}=\frac{1}{2}(\gamma+\lambda \mathbb{k}), \mathbb{b}=\mathbb{p}=0, c_{0}=0, \mathbb{C}_{1}=\mathbb{C}_{3}=0$ and $\mathbb{C}_{2}=\mathbb{k}$. The invariance conditions (3.75) imply that $c=0$ and also

$$
\mu \llbracket(\gamma+\lambda \mathbb{k})=(\gamma+\lambda \mathbb{k}) \mathbb{q} \quad \text { and } \quad a \mathbb{k}=q \mathbb{k} \overline{\mathbb{q}} .
$$

It is very similar to the previous Lie superalgebra, except that here $\gamma \neq 1$. Lemma 3 says now that either $a=|\mathbb{q}|^{2}$ and $q=q_{4}+q_{3} \mathbb{k}$ or $a=-|\mathfrak{q}|^{2}$ and $\mathfrak{q}=q_{1} \stackrel{\AA}{ }+q_{2} \AA$. In particular, since $q \neq 0, a \neq 0$. From the expressions for the automorphisms $(A, \mu)$ of $\mathfrak{k}$, we see that $\mu=1$. This means that the first equation says $q$ commutes with $\gamma+\lambda \mathbb{k}$. If $\lambda=0$, this condition is vacuous, but if $\lambda \neq 0$, then it forces $\mathbb{q}=q_{4}+q_{3} \mathbb{k}$ and hence $a=|\mathbb{q}|^{2}$.

In summary, for $\lambda \neq 0$ we have that $(A, \mu, \mathbb{q})$ takes the form

$$
A=\left(\begin{array}{cc}
|q|^{2} & 0 \\
0 & d
\end{array}\right), \quad \mu=1 \quad \text { and } \quad \mathbb{q}=q_{4}+q_{3} \mathbb{k}
$$

whereas if $\lambda=0$ it can also take the form

$$
A=\left(\begin{array}{cc}
-|q|^{2} & 0 \\
0 & d
\end{array}\right), \quad \mu=1 \quad \text { and } \quad \mathbb{q}=q_{1} \stackrel{\imath}{ }+q_{2} \stackrel{\jmath}{ } .
$$

The next Lie superalgebra is based on the kinematical Lie algebra $\mathrm{K} 4_{\chi}$, whose automorphisms $(A, \mu)$ take the form

$$
A=\left(\begin{array}{cc}
a & b \\
-b & a
\end{array}\right) \quad \text { and } \quad \mu=1
$$

for generic $\chi$, whereas if $\chi=0$, then they can also be of the form

$$
A=\left(\begin{array}{cc}
a & b \\
b & -a
\end{array}\right) \quad \text { and } \quad \mu=-1 .
$$

\subsubsection{Automorphisms of Lie superalgebra $S 11_{\chi}$}

Here $\mathfrak{h}=\frac{1}{2}(\chi+i), \mathfrak{b}=\mathbb{p}=0, c_{0}=0, \mathbb{C}_{1}=0, \mathbb{C}_{2}=\AA$ and $\mathbb{C}_{3}=\mathbb{k}$. The invariance conditions $(3.75)$ reduce to

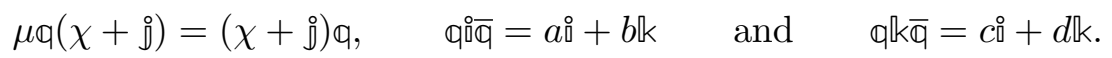


The last two equations are solved via Lemma 4: either $\mathbb{q}=q_{4}+q_{2} \AA$ or else $\mathbb{q}=q_{1} \AA+q_{3} \mathbb{k}$. This latter case can only happen when $\chi=0$. Substituting these possible expressions for $\mathbb{q}$ in the last two equations, we determine the entries of the matrix $A$.

In summary, $(A, \mu, \mathbb{q})$ takes the form

$$
A=\left(\begin{array}{cc}
q_{4}^{2}-q_{2}^{2}-2 q_{2} q_{4} \\
2 q_{2} q_{4} & q_{4}^{2}-q_{2}^{2}
\end{array}\right), \quad \mu=1 \quad \text { and } \quad \mathbb{q}=q_{4}+q_{2} \AA
$$

and (only) if $\chi=0$ it can also take the form

$$
A=\left(\begin{array}{cc}
q_{1}^{2}-q_{3}^{2} & 2 q_{1} q_{3} \\
2 q_{1} q_{3} & q_{3}^{2}-q_{1}^{2}
\end{array}\right), \quad \mu=-1 \quad \text { and } \quad \mathbb{q}=q_{1} \stackrel{\AA}{\mathbb{1}}+q_{3} \mathbb{k} .
$$

The next Lie superalgebra is the supersymmetric extension of the kinematical Lie algebra $\mathrm{K} 5$, whose automorphisms $(A, \mu)$ are of the form

$$
A=\left(\begin{array}{ll}
a & 0 \\
c & a
\end{array}\right) \quad \text { and } \quad \mu=1
$$

\subsubsection{Automorphisms of Lie superalgebra $S 12_{\lambda}$}

Here $\mathbb{h}=\frac{1}{2}(1+\lambda \mathbb{k}), \mathbb{b}=\mathbb{p}=0, c_{0}=0, \mathbb{C}_{1}=\mathbb{C}_{2}=0$ and $\mathbb{C}_{3}=\mathbb{k}$. The invariance conditions $(3.75)$ reduce to

$$
\mathbb{q} \text { h }=\text { thq } \quad \text { and } \quad a \mathbb{k}=\mathbb{q} k \bar{q} .
$$

The second equation is solved via Lemma 3, which says that either $a=|\mathfrak{q}|^{2}$ and $\mathbb{q}=q_{4}+q_{3} \mathbb{k}$ or $a=-|\mathbb{q}|^{2}$ and $\mathbb{q}=q_{1} \AA+q_{2} \AA$. The first equation is identically satisfied if $\lambda=0$, but otherwise it forces $\mathbb{q}=q_{4}+q_{3} \mathbb{k}$ and hence $a=|\mathbb{q}|^{2}$. In summary, for general $\lambda$, an automorphism $(A, \mu, q)$ takes the form

$$
A=\left(\begin{array}{cc}
|\mathbb{q}|^{2} & 0 \\
c & |\mathbb{q}|^{2}
\end{array}\right), \quad \mu=1 \quad \text { and } \quad \mathbb{q}=q_{4}+q_{3} \mathbb{k},
$$

whereas if $\lambda=0$, it can also take the form

$$
A=\left(\begin{array}{cc}
-|\mathbb{q}|^{2} & 0 \\
c & -|q|^{2}
\end{array}\right), \quad \mu=1 \quad \text { and } \quad \mathbb{q}=q_{1} \AA+q_{2} \AA .
$$

The next Lie superalgebra is the supersymmetric extension of the Carroll algebra, whose automorphisms $(A, \mu)$ take the form

$$
A=\left(\begin{array}{ll}
a & b \\
c & d
\end{array}\right) \quad \text { and } \quad \mu=a d-b c .
$$




\subsubsection{Automorphisms of Lie superalgebra S13}

Here $\mathbb{h}=\mathbb{b}=\mathfrak{p}=0, c_{0}=1$ and $\mathbb{C}_{1}=\mathbb{C}_{2}=\mathbb{C}_{3}=0$. The invariance conditions (3.75) reduce to a single condition: $a d-b c=|\mathbb{q}|^{2}$. The automorphisms $(A, \mu, \mathbb{q})$ are of the form

$$
A=\left(\begin{array}{ll}
a & b \\
c & d
\end{array}\right), \quad \mu=a d-b c=|\mathbb{q}|^{2} \quad \text { and } \quad q \in \mathbb{H}^{\times} .
$$

The next Lie superalgebra is the Poincaré superalgebra whose ( $\mathfrak{r}$-fixing) automorphisms $(A, \mu)$ can take one of two possible forms:

$$
\begin{aligned}
A & =\left(\begin{array}{ll}
1 & 0 \\
c & d
\end{array}\right) \quad \text { and } \quad \mu \\
\text { or } \quad A & =\left(\begin{array}{cc}
-1 & 0 \\
c & d
\end{array}\right) \text { and } \quad \mu=-d .
\end{aligned}
$$

\subsubsection{Automorphisms of Lie superalgebra S14}

Here $\mathbb{h}=\mathfrak{p}=0, \mathbb{b}=\frac{1}{2} \mathbb{k}, c_{0}=1, \mathbb{C}_{1}=\mathbb{C}_{2}=0$ and $\mathbb{C}_{3}=\mathbb{k}$. The invariance conditions (3.75) translate into

$$
\pm \mathbb{q} \mathbb{k}=\mathbb{k} \mathfrak{q}, \quad d= \pm|\mathbb{q}|^{2} \quad \text { and } \quad d \mathbb{k}=\mathbb{q} \mathbb{k} \overline{\mathfrak{q}},
$$

where the signs are correlated and the last equation follows from the first two.

Choosing the plus sign, $\mathbb{q} \mathbb{k}=\mathbb{k} \mathfrak{q}$, so that $\mathbb{q}=q_{4}+q_{3} \mathbb{k}$ and $d=|\mathbb{q}|^{2}$, whereas choosing the minus sign, $\mathfrak{q} \mathbb{k}=-\mathbb{k} \mathfrak{q}$, so that $\mathfrak{q}=q_{1} \AA+q_{2} \AA$ and $d=-|\mathfrak{q}|^{2}$.

In summary, automorphisms $(A, \mu, q)$ of the Poincaré superalgebra take the form

$$
\begin{aligned}
A & =\left(\begin{array}{cc}
1 & 0 \\
c & |\mathbb{q}|^{2}
\end{array}\right), \quad \mu=|\mathbb{q}|^{2} \quad \text { and } \quad \mathbb{q}=q_{4}+q_{3} \mathbb{k} \\
\text { or } \quad A & =\left(\begin{array}{cc}
-1 & 0 \\
c & -|q|^{2}
\end{array}\right), \quad \mu=|\mathbb{q}|^{2} \quad \text { and } \quad \mathbb{q}=q_{1} \AA+q_{2} \AA .
\end{aligned}
$$

The next Lie superalgebra is the AdS superalgebra, whose ( $\mathfrak{r}$-fixing) automorphisms $(A, \mu)$ are of the form

$$
A=\left(\begin{array}{cc}
a & b \\
\mp b & \pm a
\end{array}\right) \quad \text { and } \quad \mu= \pm 1
$$

where $a^{2}+b^{2}=1$.

\subsubsection{Automorphisms of Lie superalgebra S15}

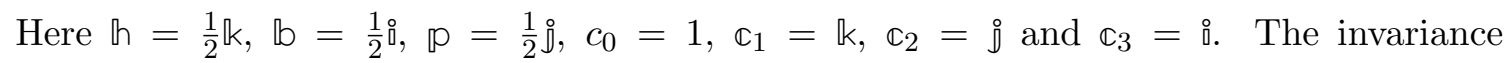
conditions (3.75) include $\mu=|q|^{2}$, which forces $\mu=1$. Taking this into account, another of the invariance conditions $(3.75)$ is $\mathbb{q} \mathbb{k}=\mathbb{k} \mathfrak{q}$, which together with $|\mathbb{q}|=1$, forces $\mathbb{q}=e^{\theta \mathbb{k}}$. The remaining invariance conditions are

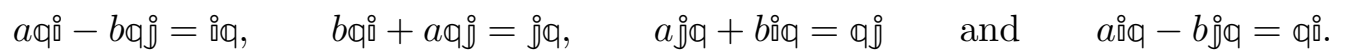


Given the expression for $\mathbb{q}$, these are solved by $a=\cos 2 \theta$ and $b=\sin 2 \theta$. In summary, the (r-fixing) automorphisms $(A, \mu, \mathbb{q})$ of the AdS superalgebra are of the form

$$
A=\left(\begin{array}{cc}
\cos 2 \theta & \sin 2 \theta \\
-\sin 2 \theta & \cos 2 \theta
\end{array}\right), \quad \mu=1 \quad \text { and } \quad \mathbb{q}=e^{\theta \mathbb{k}} .
$$

The next three Lie superalgebras in table 4 are supersymmetric extensions of the kinematical Lie algebra $\mathrm{K} 12$ in table 2 , whose $\mathfrak{r}$-fixing automorphisms $(A, \mu)$ take the following form:

$$
A=\left(\begin{array}{cc}
1 & 0 \\
0 & \pm 1
\end{array}\right) \quad \text { and } \quad \mu \in \mathbb{R}^{\times} .
$$

\subsubsection{Automorphisms of Lie superalgebra S16}

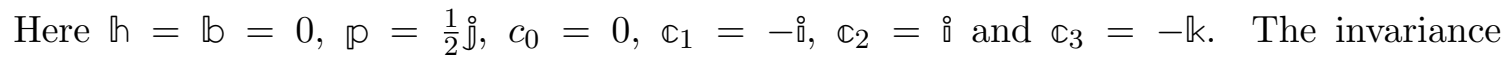
conditions (3.75) reduce to

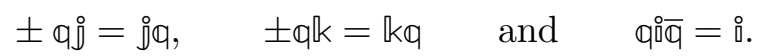

It follows from the last equation that $|q|=1$ and hence that $q q^{\circ}=\AA q$. Depending on the (correlated) signs of the first two equations, we find that, for the plus sign, $\mathbb{q}$ commutes with $\stackrel{\AA}{\AA}, \AA$ and $\mathbb{k}$ and hence $q \in \mathbb{R}$, but since $|\mathbb{q}|=1$, we must have $\mathbb{q}= \pm 1$. For the minus sign, we find that $\mathbb{q}$ commutes with $i$ but anticommutes with $₫$ and $\mathbb{k}$, so that $\mathbb{q}= \pm \AA$, after taking into account that $|\mathbb{q}|=1$. In summary, the automorphisms $(A, \mu, \mathbb{q})$ of this Lie superalgebra take one of two possible forms:

$$
\begin{array}{rlrl}
A & =\left(\begin{array}{ll}
1 & 0 \\
0 & 1
\end{array}\right), \quad \mu \in \mathbb{R}^{\times} \quad \text { and } & \mathbb{q}= \pm 1 \\
\text { or } & A=\left(\begin{array}{cc}
1 & 0 \\
0 & -1
\end{array}\right), \quad \mu \in \mathbb{R}^{\times} \quad \text { and } \quad \mathbb{q}= \pm \stackrel{\circ}{ }
\end{array}
$$

\subsubsection{Automorphisms of Lie superalgebra S17}

Here $\mathbb{h}=\mathfrak{p}=0, \mathbb{b}=\frac{1}{2}, c_{0}=1$ and $\mathbb{C}_{1}=\mathbb{C}_{2}=\mathbb{C}_{3}=0$. There is only one invariance condition: namely, $\mu=\left.|q|\right|^{2}$, and hence the automorphisms $(A, \mu, \mathfrak{q})$ take the form

$$
A=\left(\begin{array}{cc}
1 & 0 \\
0 & \pm 1
\end{array}\right), \quad \mu=|q|^{2} \quad \text { and } \quad \llbracket \in \mathbb{H}^{\times} .
$$

\subsubsection{Automorphisms of Lie superalgebra S18}

Here $\mathbb{h}=\frac{1}{2} \mathbb{k}, \mathbb{b}=\frac{1}{2}, \mathbb{p}=0, c_{0}=1, \mathbb{C}_{1}=\mathbb{C}_{3}=0$ and $\mathbb{C}_{2}=\mathbb{k}$. The invariance conditions (3.75) reduce to

$$
\mu \mathbb{q} \mathbb{k}=\mathbb{k} \mathfrak{q}, \quad \mu=|\mathbb{q}|^{2} \quad \text { and } \quad \mathbb{k}=\mathbb{q} \mathbb{k} \overline{\mathbb{q}} .
$$


From the first equation we see that $\mu= \pm 1$, but from the second it must be positive, so $\mu=1$, which says implies that $|\mathbb{q}|=1$ and hence that $\mathbb{q}$ commutes with $\mathbb{k}$. In summary, the typical automorphism $(A, \mu, q)$ takes the form

$$
A=\left(\begin{array}{cc}
1 & 0 \\
0 & \pm 1
\end{array}\right), \quad \mu=1 \quad \text { and } \quad \mathbb{q}=e^{\theta \mathbb{k}} .
$$

The next four Lie superalgebras in table 4 are supersymmetric extensions of the kinematical Lie algebra $\mathrm{K} 13$ in table 2 , whose typical $\mathfrak{r}$-fixing automorphisms $(A, \mu)$ take the form

$$
A=\left(\begin{array}{cc}
1 & 0 \\
0 & \pm 1
\end{array}\right) \quad \text { and } \quad \mu \in \mathbb{R}^{\times}
$$

\subsubsection{Automorphisms of Lie superalgebra S19}

Here $\mathbb{h}=\mathbb{k}, \mathfrak{b}=0, \mathbb{p}=\frac{1}{2}, c_{0}=1, \mathbb{C}_{1}=\mathbb{C}_{3}=\mathbb{k}$ and $\mathbb{C}_{2}=-\mathbb{k}$. The invariance conditions (3.75) are given by

$$
\mu q \mathbb{k}=\mathbb{k} q, \quad \mu=|\mathbb{q}|^{2} \quad \text { and } \quad d q=\mathbb{q} .
$$

The last equation says that $d=1$, whereas the first says that $\mu= \pm 1$, but from the second equation it is positive and thus $\mu=1$. This also means $|\mathfrak{q}|=1$ and that $q \mathbb{k} k=\mathbb{k} q$. In summary, the typical automorphism $(A, \mu, \mathfrak{q})$ of $\mathfrak{s}$ takes the form

$$
A=\left(\begin{array}{ll}
1 & 0 \\
0 & 1
\end{array}\right), \quad \mu=1 \quad \text { and } \quad \mathbb{q}=e^{\theta \mathbb{k}} .
$$

\subsubsection{Automorphisms of Lie superalgebra S20}

Here $\mathbb{h}=\mathfrak{b}=0, \mathbb{p}=\frac{1}{2}, c_{0}=1, \mathbb{C}_{1}=\mathbb{C}_{2}=\mathbb{C}_{3}=0$. The invariance conditions (3.75) are given by

$$
d q=\mathbb{q} \quad \text { and } \quad \mu=|q|^{2} .
$$

The first equation simply sets $d=1$ and, in summary, the typical automorphism of $\mathfrak{s}$ is takes the form

$$
A=\left(\begin{array}{ll}
1 & 0 \\
0 & 1
\end{array}\right), \quad \mu=|q|^{2} \quad \text { and } \quad \mathbb{q} \in \mathbb{H}^{\times} .
$$

\subsubsection{Automorphisms of Lie superalgebra S21}

Here $\mathfrak{h}=\mathfrak{p}=0, \mathbb{b}=\frac{1}{2}, c_{0}=1$ and $\mathbb{C}_{1}=\mathbb{C}_{2}=\mathbb{C}_{3}=0$. The only invariance condition is $\mu=|\mathbb{q}|^{2}$, so that the typical automorphism $(A, \mu, \mathbb{q})$ takes the form

$$
A=\left(\begin{array}{cc}
1 & 0 \\
0 & \pm 1
\end{array}\right), \quad \mu=|q|^{2} \quad \text { and } \quad \mathbb{q} \in \mathbb{H}^{\times} \text {. }
$$




\subsubsection{Automorphisms of Lie superalgebra S22}

Here $\mathbb{h}=\frac{1}{2} \mathbb{k}, \mathbb{b}=\frac{1}{2}, \mathbb{p}=0, c_{0}=1, \mathbb{C}_{1}=\mathbb{C}_{3}=0$ and $\mathbb{C}_{2}=\mathbb{k}$. The invariance conditions (3.75) reduce to

$$
\mu q \mathbb{k}=\mathbb{k q} \quad \text { and } \quad \mu=|\mathbb{q}|^{2} .
$$

The first equation says that $\mu= \pm 1$, but the second equation says it is positive, so that $\mu=1$ and $|\mathbb{q}|=1$. Furthermore, $\mathbb{q}$ commutes with $\mathbb{k}$, so that $\mathbb{q}=e^{\theta \mathbb{k}}$. In summary, the typical automorphism $(A, \mu, q)$ takes the form

$$
A=\left(\begin{array}{cc}
1 & 0 \\
0 & \pm 1
\end{array}\right), \quad \mu=1 \quad \text { and } \quad \mathbb{q}=e^{\theta \mathbb{k}}
$$

The next six Lie superalgebras in table 4 are supersymmetric extensions of the kinematical Lie algebra $\mathrm{K} 14$ in table 2 , whose $\mathfrak{r}$-fixing automorphisms $(A, \mu)$ take the form

$$
A=\left(\begin{array}{ll}
1 & 0 \\
0 & d
\end{array}\right) \quad \text { and } \quad \mu \in \mathbb{R}^{\times} .
$$

\subsubsection{Automorphisms of Lie superalgebra S23}

Here $\mathbb{h}=\mathbb{k}, \mathfrak{b}=\mathbb{p}=0, c_{0}=0, \mathbb{C}_{1}=\mathbb{C}_{2}=0$ and $\mathbb{C}_{3}=\mathbb{k}$. The invariance conditions $(3.75)$ reduce to

$$
\mu \mathbb{q} k=\mathbb{k} \mathbb{q} \quad \text { and } \quad d \mathbb{k}=\mathbb{q} \mathbb{k} \overline{\mathbb{q}} .
$$

The first equation says that $\mu= \pm 1$, so that $\pm \mathbb{q k}=\mathbb{k q}$. The second equation follows from Lemma 3: either $d=|\mathfrak{q}|^{2}$ and hence $\mathbb{q}=q_{4}+q_{3} \mathbb{k}$ or $d=-|\mathfrak{q}|^{2}$ and hence $\mathbb{q}=q_{1} \AA+q_{2} \AA$. In summary, the typical automorphism $(A, \mu, q)$ takes one of two possible forms:

$$
\begin{aligned}
A & =\left(\begin{array}{cc}
1 & 0 \\
0 & |\mathbb{q}|^{2}
\end{array}\right), \quad \mu=1 \quad \text { and } \quad \mathbb{q}=q_{4}+q_{3} \mathbb{k} \\
\text { or } \quad A & =\left(\begin{array}{cc}
1 & 0 \\
0 & -|\mathbb{q}|^{2}
\end{array}\right), \quad \mu=-1 \quad \text { and } \quad \mathbb{q}=q_{1} \AA+q_{2} \AA .
\end{aligned}
$$

\subsubsection{Automorphisms of Lie superalgebra S24}

Here $\mathbb{h}=\mathbb{b}=\mathfrak{p}=0, c_{0}=0, \mathbb{C}_{1}=\mathbb{C}_{2}=0$ and $\mathbb{C}_{3}=\mathbb{k}$. Hence the only invariance condition is $d \mathbb{k}=\mathbb{q} \mathbb{k} \overline{\mathfrak{q}}$. Lemma 3 says that either $d=|\mathbb{q}|^{2}$ and hence $\mathbb{q}=q_{4}+q_{3} \mathbb{k}$ or else $d=-|\mathbb{q}|^{2}$ and hence $\mathbb{q}=q_{1} \AA+q_{2} \AA$. In summary, the typical automorphism $(A, \mu, q)$ takes one of two possible forms:

$$
\begin{aligned}
& A=\left(\begin{array}{cc}
1 & 0 \\
0 & |\mathbb{q}|^{2}
\end{array}\right), \quad \mu \in \mathbb{R}^{\times} \quad \text { and } \quad \mathbb{q}=q_{4}+q_{3} \mathbb{k} \\
& \text { or } \quad A=\left(\begin{array}{cc}
1 & 0 \\
0 & -|\mathbb{q}|^{2}
\end{array}\right), \quad \mu \in \mathbb{R}^{\times} \quad \text { and } \quad \mathbb{q}=q_{1} \AA+q_{2} \AA \text {. }
\end{aligned}
$$




\subsubsection{Automorphisms of Lie superalgebra S25}

Here $\mathbb{h}=\mathbb{b}=\mathfrak{p}=0, c_{0}=1$ and $\mathbb{C}_{1}=\mathbb{C}_{2}=\mathbb{C}_{3}=0$, so that the only invariance condition is $\mu=|\mathbb{q}|^{2}$. In summary, the typical automorphism $(A, \mu, \mathbb{q})$ takes the form

$$
A=\left(\begin{array}{ll}
1 & 0 \\
0 & d
\end{array}\right), \quad \mu=|\mathbb{q}|^{2} \quad \text { and } \quad \mathbb{q} \in \mathbb{H}^{\times} .
$$

\subsubsection{Automorphisms of Lie superalgebra S26}

Here $\mathbb{h}=\mathbb{b}=\mathfrak{p}=0, c_{0}=1, \mathbb{C}_{1}=\mathbb{C}_{2}=0$ and $\mathbb{C}_{3}=\mathbb{k}$, so that there are two conditions in $(3.75)$ :

$$
\mu=|\mathbb{q}|^{2} \quad \text { and } \quad d \mathbb{k}=\mathbb{q} \mathbb{k} \overline{\mathbb{q}} .
$$

The second equation can be solved via Lemma 3: either $d=|\mathfrak{q}|^{2}$ and $\mathbb{q}=q_{4}+q_{3}$ k or $d=-|\mathfrak{q}|^{2}$ and $\mathfrak{q}=q_{1} \AA+q_{2} \AA$. In summary, the automorphisms $(A, \mu, \mathbb{q})$ take one of two possible forms:

$$
\begin{aligned}
& A=\left(\begin{array}{cc}
1 & 0 \\
0 & |\mathbb{q}|^{2}
\end{array}\right), \quad \mu=|\mathbb{q}|^{2} \quad \text { and } \quad \mathbb{q}=q_{4}+q_{3} \mathbb{k} \\
& \text { or } \quad A=\left(\begin{array}{cc}
1 & 0 \\
0 & -|\mathbb{q}|^{2}
\end{array}\right), \quad \mu=|\mathbb{q}|^{2} \quad \text { and } \quad \mathbb{q}=q_{1} \stackrel{\mathbb{q}}{ }+q_{2} \AA \text {. }
\end{aligned}
$$

\subsubsection{Automorphisms of Lie superalgebra S27}

Here $\mathfrak{h}=\mathbb{p}=0, \mathfrak{b}=\frac{1}{2}, c_{0}=1$ and $\mathbb{C}_{1}=\mathbb{C}_{2}=\mathbb{C}_{3}=0$, so that the only invariance condition is $\mu=|\mathbb{q}|^{2}$. Therefore the typical automorphism $(A, \mu, \mathbb{q})$ takes the form

$$
A=\left(\begin{array}{ll}
1 & 0 \\
0 & d
\end{array}\right), \quad \mu=|q|^{2} \quad \text { and } \quad q \in \mathbb{H}^{\times} .
$$

\subsubsection{Automorphisms of Lie superalgebra S28}

Here $\mathfrak{h}=\mathbb{b}=\frac{1}{2}, \mathbb{p}=0, c_{0}=1, \mathbb{C}_{1}=\mathbb{C}_{3}=0$ and $\mathbb{C}_{2}=\mathbb{k}$. The invariance conditions (3.75) reduce to the following:

$$
\mu=|\mathbb{q}|^{2}, \quad \mu q=\mathbb{q} \quad \text { and } \quad \mathbb{k}=\mathbb{q} \mathbb{k} \bar{q} .
$$

From the second equation we see that $\mu=1$, so that from the first $|q|=1$ and hence $\mathbb{k} \mathbb{q}=\mathbb{q} \mathbb{k}$, so that $\mathbb{q}=e^{\theta \mathbb{k}}$. In summary, the typical automorphism $(A, \mu, \mathbb{p})$ takes the form

$$
A=\left(\begin{array}{ll}
1 & 0 \\
0 & d
\end{array}\right), \quad \mu=1 \quad \text { and } \quad \mathbb{q}=e^{\theta \mathbb{k}} .
$$

The next four Lie superalgebras in table 4 are supersymmetric extensions of the kinematical Lie algebra $\mathrm{K} 15$ in table 2, whose $\mathfrak{r}$-fixing automorphisms $(A, \mu)$ take the form

$$
A=\left(\begin{array}{cc}
a & 0 \\
c & a^{2}
\end{array}\right) \quad \text { and } \quad \mu \in \mathbb{R}^{\times} .
$$




\subsubsection{Automorphisms of Lie superalgebra S29}

Here $\mathbb{b}=\mathfrak{p}=0, \mathbb{h}=\mathbb{k}, c_{0}=0, \mathbb{C}_{1}=\mathbb{C}_{2}=0$ and $\mathbb{C}_{3}=\mathbb{k}$. The invariance conditions (3.75) result in

$$
\mu q \mathbb{k}=\mathbb{k} \mathfrak{q} \quad \text { and } \quad a^{2} \mathbb{k}=\mathbb{q} \mathbb{k} \bar{q} .
$$

Taking the norm of the first equation, we see that $\mu= \pm 1$, and of the second equation, $a^{2}=|q|^{2}$. This then says that $\mathbb{q}$ commutes with $\mathbb{k}$, so that $\mu=1$ and $\mathbb{q}=q_{4}+q_{3} \mathbb{k}$. In summary, the typical automorphism $(A, \mu, \mathbb{q})$ takes the form

$$
A=\left(\begin{array}{cc} 
\pm|\mathbb{q}| & 0 \\
c & |\mathbb{q}|^{2}
\end{array}\right), \quad \mu=1 \quad \text { and } \quad \mathbb{q}=q_{4}+q_{3} \mathbb{k} .
$$

\subsubsection{Automorphisms of Lie superalgebra S30}

Here $\mathbb{h}=\mathbb{b}=\mathbb{p}=0, c_{0}=0, \mathbb{C}_{1}=\mathbb{C}_{2}=0$ and $\mathbb{C}_{3}=\mathbb{k}$. The only invariance condition is $a^{2} \mathbb{k}=\mathfrak{q} \mathbb{k} \overline{\mathbb{q}}$. Taking the norm, $a^{2}=|\mathfrak{q}|^{2}$ and hence $\mathbb{k q}=\mathfrak{q} \mathbb{k}$ and thus $\mathbb{q}=q_{4}+q_{3} \mathbb{k}$. Hence the typical automorphism $(A, \mu, \mathbb{q})$ takes the form

$$
A=\left(\begin{array}{cc} 
\pm|\mathbb{q}| & 0 \\
c & |\mathbb{q}|^{2}
\end{array}\right), \quad \mu \in \mathbb{R}^{\times} \quad \text { and } \quad \mathbb{q}=q_{4}+q_{3} \mathbb{k} .
$$

\subsubsection{Automorphisms of Lie superalgebra S31}

Here $\mathfrak{h}=\mathbb{b}=\mathfrak{p}=0, c_{0}=1$ and $\mathbb{C}_{1}=\mathbb{C}_{2}=\mathbb{C}_{3}=0$, so that the only invariance condition is $\mu=|\mathbb{q}|^{2}$. In summary, the typical automorphism $(A, \mu, \mathbb{q})$ takes the form

$$
A=\left(\begin{array}{cc}
a & 0 \\
c & a^{2}
\end{array}\right), \quad \mu=|q|^{2} \quad \text { and } \quad \mathbb{q} \in \mathbb{H}^{\times} .
$$

\subsubsection{Automorphisms of Lie superalgebra S32}

Here $\mathfrak{h}=\mathbb{b}=\mathfrak{p}=0, c_{0}=1, \mathbb{C}_{1}=\mathbb{C}_{2}=0$ and $\mathbb{C}_{3}=\mathbb{k}$, so that there are two invariance conditions:

$$
\mu=|\mathbb{q}|^{2} \quad \text { and } \quad a^{2} \mathbb{k}=\mathbb{q} k \overline{\mathbb{q}} .
$$

The second shows that $a^{2}=|\mathbb{q}|^{2}$ and hence $\mathbb{q}$ commutes with $\mathbb{k}$, so that $\mathbb{q}=q_{4}+q_{3} \mathbb{k}$. In summary, the typical automorphism $(A, \mu, \mathbb{q})$ takes the form

$$
A=\left(\begin{array}{cc} 
\pm|q| & 0 \\
c & |\mathbb{q}|^{2}
\end{array}\right), \quad \mu=|\mathbb{q}|^{2} \quad \text { and } \quad \mathbb{q}=q_{4}+q_{3} \mathbb{k} .
$$

The next Lie superalgebra in table 4 is a one-parameter family of supersymmetric extensions of the kinematical Lie algebra $\mathrm{K} 16$ in table 2, whose $\mathfrak{r}$-fixing automorphisms $(A, \mu)$ take the form

$$
A=\left(\begin{array}{ll}
1 & 0 \\
0 & d
\end{array}\right) \quad \text { and } \quad \mu=1
$$




\subsubsection{Automorphisms of Lie superalgebra S33}

Here $\mathbb{h}=\frac{1}{2}(1+\lambda \mathbb{k}), \mathbb{b}=\mathfrak{p}=0, c_{0}=0, \mathbb{C}_{1}=\mathbb{C}_{2}=0$ and $\mathbb{C}_{3}=\mathbb{k}$. There are two invariance conditions:

$$
\mathbb{q}(1+\lambda \mathbb{k})=(1+\lambda \mathbb{k}) \mathfrak{q} \quad \text { and } \quad d \mathbb{k}=\mathbb{q} \mathbb{k} \overline{\mathbb{q}} .
$$

For the second equation we use Lemma 3 and for the first equation we must distinguish between $\lambda=0$ and $\lambda \neq 0$. In the latter case, we have that $\mathbb{q}=q_{4}+q_{3} \mathbb{k}$ so that only the $d=|\mathbb{q}|^{2}$ of the lemma survives. If $\lambda=0$, both branches survive. In summary, for $\lambda \neq 0$, the typical automorphism $(A, \mu, \mathfrak{q})$ takes the form

$$
A=\left(\begin{array}{cc}
1 & 0 \\
0 & |q|^{2}
\end{array}\right), \quad \mu=1 \quad \text { and } \quad \mathbb{q}=q_{4}+q_{3} \mathbb{k}
$$

whereas if $\lambda=0$ we have additional automorphisms of the form

$$
A=\left(\begin{array}{cc}
1 & 0 \\
0 & -|q|^{2}
\end{array}\right), \quad \mu=1 \quad \text { and } \quad \mathbb{q}=q_{1} \AA+q_{2} \AA
$$

The next Lie superalgebra in table 4 is the supersymmetric extension of the kinematical Lie algebra $\mathrm{K} 17$ in table 2 , whose $\mathfrak{r}$-fixing automorphisms $(A, \mu)$ take the form

$$
A=\left(\begin{array}{cc}
a & 0 \\
c & a^{2}
\end{array}\right) \quad \text { and } \quad \mu=a .
$$

\subsubsection{Automorphisms of Lie superalgebra S34}

Here $\mathbb{h}=\frac{1}{2} \mathbb{k}, \mathfrak{b}=\mathfrak{p}=0, c_{0}=0, \mathbb{C}_{1}=\mathbb{C}_{2}=0$ and $\mathbb{C}_{3}=\mathbb{k}$. The invariance conditions are

$$
a q \mathbb{k}=\mathbb{k} \mathbb{q} \quad \text { and } \quad a^{2} \mathbb{k}=\mathbb{q} \mathbb{k} \overline{\mathbb{q}} .
$$

Taking norms of the first equation gives $a= \pm 1$ and hence $\pm \mathbb{q} k=\mathbb{k} \mathfrak{q}$ and of the second equation $a^{2}=|\mathfrak{q}|^{2}$ and hence $\mathbb{q} \mathbb{k}=\mathbb{k q}$. This shows that $a=1$ and hence $|\mathfrak{q}|=1$, so that $\mathbb{q}=e^{\theta \mathbb{k}}$. In summary, the typical automorphism $(A, \mu, \mathbb{q})$ takes the form

$$
A=\left(\begin{array}{ll}
1 & 0 \\
c & 1
\end{array}\right), \quad \mu=1 \quad \text { and } \quad \mathbb{q}=e^{\theta \mathbb{k}} .
$$

The last Lie superalgebra in table 4 is a one-parameter family of supersymmetric extensions of the kinematical Lie algebra $\mathrm{K} 18$ in table 2, whose $\mathfrak{r}$-fixing automorphisms $(A, \mu)$ take the form

$$
A=\left(\begin{array}{cc}
a & 0 \\
0 & a^{2}
\end{array}\right) \quad \text { and } \quad \mu=1
$$




\subsubsection{Automorphisms of Lie superalgebra S35}

Here $\mathfrak{h}=1+\lambda \mathbb{k}, \mathbb{b}=\mathfrak{p}=0, c_{0}=0, \mathbb{C}_{1}=\mathbb{C}_{2}=0$ and $\mathbb{C}_{3}=\mathbb{k}$. The invariance conditions (3.75) reduce to

$$
\mathbb{q}(1+\lambda \mathbb{k})=(1+\lambda \mathbb{k}) \mathbb{q} \quad \text { and } \quad a^{2} \mathbb{k}=\mathbb{q} \mathbb{k} \overline{\mathbb{q}} .
$$

Taking the norm of the second equation, $a^{2}=|\mathfrak{q}|^{2}$ so that $\mathbb{q} k=k \mathbb{q}$ and hence $\mathfrak{q}=q_{4}+q_{3} \mathbb{k}$. This also solves the first equation, independently of the value of $\lambda$. In summary, the typical automorphism $(A, \mu, \mathbb{q})$ takes the form

$$
A=\left(\begin{array}{cc} 
\pm|\mathbb{q}| & 0 \\
0 & |\mathbb{q}|^{2}
\end{array}\right), \quad \mu=1 \quad \text { and } \quad \mathbb{q}=q_{4}+q_{3} \mathbb{k} .
$$

\subsubsection{Summary}

Tables 8 and 9 summarise the above discussion and lists the typical automorphisms of each of the Lie superalgebras in table 4.

\section{Homogeneous superspaces}

In this section, we classify the simply-connected (4|4)-dimensional homogeneous kinematical and aristotelian superspaces. We start by classifying the super Lie pairs associated with the kinematical Lie superalgebras. After determining the super Lie pairs, we select those super Lie pairs $(\mathfrak{s}, \mathfrak{h})$ which are effective in a basis where $\mathfrak{h}$ is always the span of $\boldsymbol{J}$ and $\boldsymbol{B}$. In this way, the super Lie pair is uniquely characterised by writing the Lie brackets of $\mathfrak{s}$ in that basis.

Before starting with the classification of super Lie pairs, we first explain the relationship between super Lie pairs and homogeneous supermanifolds. We shall be brief and refer the reader to [18], particularly section 5 , for the details. Although the treatment in that paper is phrased in the context of spin manifolds, the results are more general and apply to the homogeneous spacetimes under consideration, even in the absence of an invariant pseudoriemannian structure.

\subsection{Homogeneous supermanifolds}

In this paper, we shall adopt the following definition for supermanifolds (see, e.g., [19]).

Definition 2. A smooth supermanifold of dimension $(m \mid n)$ is a pair $(M, \mathcal{O})$, where the body $M$ is a smooth $m$-dimensional manifold and the structure sheaf $\mathcal{O}$ is a sheaf of supercommutative superalgebras extending the sheaf $\mathrm{e}^{\infty}$ of smooth function of $M$ by the subalgebra of nilpotent elements $\mathcal{N}$; that is, we have an exact sequence of sheaves of supercommutative superalgebras:

$$
0 \longrightarrow \mathcal{N} \longrightarrow \mathcal{O} \longrightarrow \mathrm{C}^{\infty} \longrightarrow 0
$$

where for every $p \in M$, there is a neighbourhood $p \in U \subset M$ such that

$$
\mathcal{O}(U) \cong \mathcal{C}^{\infty}(U) \otimes \wedge\left[\theta^{1}, \ldots, \theta^{n}\right] .
$$




\begin{tabular}{|c|c|}
\hline S\# & Typical $(A, \mu, q) \in \mathrm{GL}(2, \mathbb{R}) \times \mathbb{R}^{\times} \times \mathbb{H}^{\times}$ \\
\hline 1 & $\left(\left(\begin{array}{cc}a & 0 \\
c & |\mathbb{q}|^{2}\end{array}\right), 1, q_{4}+q_{3} \mathbb{k}\right),\left(\left(\begin{array}{cc}a & 0 \\
c & -|\mathbb{q}|^{2}\end{array}\right),-1, q_{1} \AA+q_{2} \AA\right)$ \\
\hline 2 & $\left(\left(\begin{array}{cc}q_{4}^{2}-q_{1}^{2} & 2 q_{1} q_{4} \\
-2 q_{1} q_{4} & q_{4}^{2}-q_{1}^{2}\end{array}\right), q_{1}^{2}+q_{4}^{2}, q_{4}+q_{1} \stackrel{\emptyset}{\emptyset}\right),\left(\left(\begin{array}{cc}q_{2}^{2}-q_{3}^{2} & 2 q_{2} q_{3} \\
2 q_{2} q_{3} & q_{3}^{2}-q_{2}^{2}\end{array}\right), q_{2}^{2}+q_{3}^{2}, q_{2} \AA+q_{3} \mathbb{k}\right)$ \\
\hline 3 & $\left.\left(\begin{array}{cc}a & 0 \\
c & -|q|^{2}\end{array}\right),|q|^{2}, q_{1} \AA+q_{2} \AA\right)$ \\
\hline 4 & $\left(\left(\begin{array}{ll}a & b \\
c & d\end{array}\right),|q|^{2}, q\right)$ \\
\hline 5 & $\left.q_{1} \mathrm{\AA}\right),\left(\left(\begin{array}{cc}q_{2}^{2}-q_{3}^{2} & 2 q_{2} q_{3} \\
2 q_{2} q_{3} & q_{3}^{2}-q_{2}^{2}\end{array}\right), \mu, q_{2} \AA+q_{3} \mathbb{k}\right)$ \\
\hline 6 & $\left(\left(\begin{array}{ll}a & 0 \\
c & |q|^{2}\end{array}\right), \mu, q_{4}+q_{3} \mathbb{k}\right)$ \\
\hline 7 & $\left(\left(\begin{array}{cc}|q|^{2} & 0 \\
c & |q|^{2}\end{array}\right), 1, q_{4}+q_{3} \mathbb{k}\right),\left(\left(\begin{array}{cc}|q|^{2} & 0 \\
c & -|q|^{2}\end{array}\right),-1, q_{1} \AA+q_{2} \AA\right)$ \\
\hline 8 & $\left.\left(\begin{array}{cc}a & 0 \\
c & -|\mathbb{q}|^{2}\end{array}\right),-\frac{|\mathfrak{q}|^{2}}{a}, q_{1} \AA+q_{2} \AA\right)$ \\
\hline $9_{\gamma \neq 1, \lambda \neq 0}$ & $\left(\left(\begin{array}{cc}a & 0 \\
0 & |\mathfrak{q}|^{2}\end{array}\right), 1, q_{4}+q_{3} \mathbb{k}\right)$ \\
\hline $9_{\gamma=1, \lambda \neq 0}$ & $\left(\left(\begin{array}{cc}a & 0 \\
c & |\mathfrak{q}|^{2}\end{array}\right), 1, q_{4}+q_{3} \mathbb{k}\right)$ \\
\hline $9_{\gamma \neq 1, \lambda=0}$ & $\left(\left(\begin{array}{cc}a & 0 \\
0 & |\mathbb{q}|^{2}\end{array}\right), 1, q_{4}+q_{3} \mathbb{k}\right)$, \\
\hline $9_{\gamma=1, \lambda=0}$ & $\left(\left(\begin{array}{cc}a & 0 \\
c & |q|^{2}\end{array}\right), 1, q_{4}+q_{3} \mathbb{k}\right)$ \\
\hline $10_{\gamma, \lambda \neq 0}$ & $\left(\left(\begin{array}{cc}\left.|q|\right|^{2} & 0 \\
0 & d\end{array}\right), 1, q_{4}+q_{3} \mathbb{k}\right)$ \\
\hline $10_{\gamma, \lambda=0}$ & $\left(\left(\begin{array}{cc}|q| & 0 \\
0 & d\end{array}\right), 1, q_{4}+q_{3} \mathbb{k}\right),($ \\
\hline $11_{\chi>0}$ & $\left(\left(\begin{array}{cc}q_{4}^{2}-q_{2}^{2}-2 q_{2} q_{4} \\
2 q_{2} q_{4} & q_{4}^{2}-q_{2}^{2}\end{array}\right), 1, q_{4}\right.$ \\
\hline $11_{\chi=0}$ & $\left.q_{2} \stackrel{\jmath}{ }\right),\left(\left(\begin{array}{cc}q_{1}^{2}-q_{3}^{2} & 2 q_{1} q_{3} \\
2 q_{1} q_{3} & q_{3}^{2}-q_{1}^{2}\end{array}\right),-1, q_{1} \AA+q_{3} \mathbb{k}\right)$ \\
\hline $12_{\lambda \neq 0}$ & $\left(\left(\begin{array}{cc}|\mathfrak{q}|^{2} & 0 \\
c & \left.|q|\right|^{2}\end{array}\right), 1, q_{4}+q_{3} \mathbb{k}\right)$ \\
\hline $12_{\lambda=0}$ & 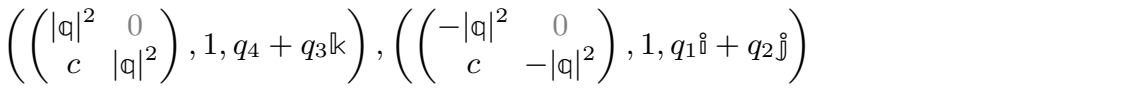 \\
\hline 13 & $\left(\left(\begin{array}{ll}a & b \\
c & d\end{array}\right), a d-b c=|q|^{2}, q\right)$ \\
\hline 14 & 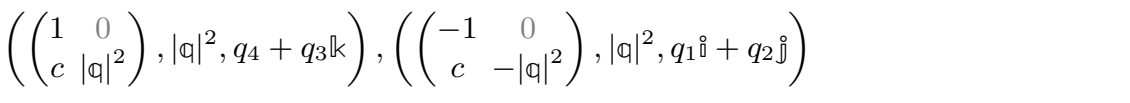 \\
\hline 15 & $\left(\left(\begin{array}{cc}\cos 2 \theta & -\sin 2 \theta \\
\sin 2 \theta & \cos 2 \theta\end{array}\right), 1, e^{\theta \mathbb{k}}\right)$ \\
\hline
\end{tabular}

Table 8. Automorphisms of kinematical Lie superalgebras. 


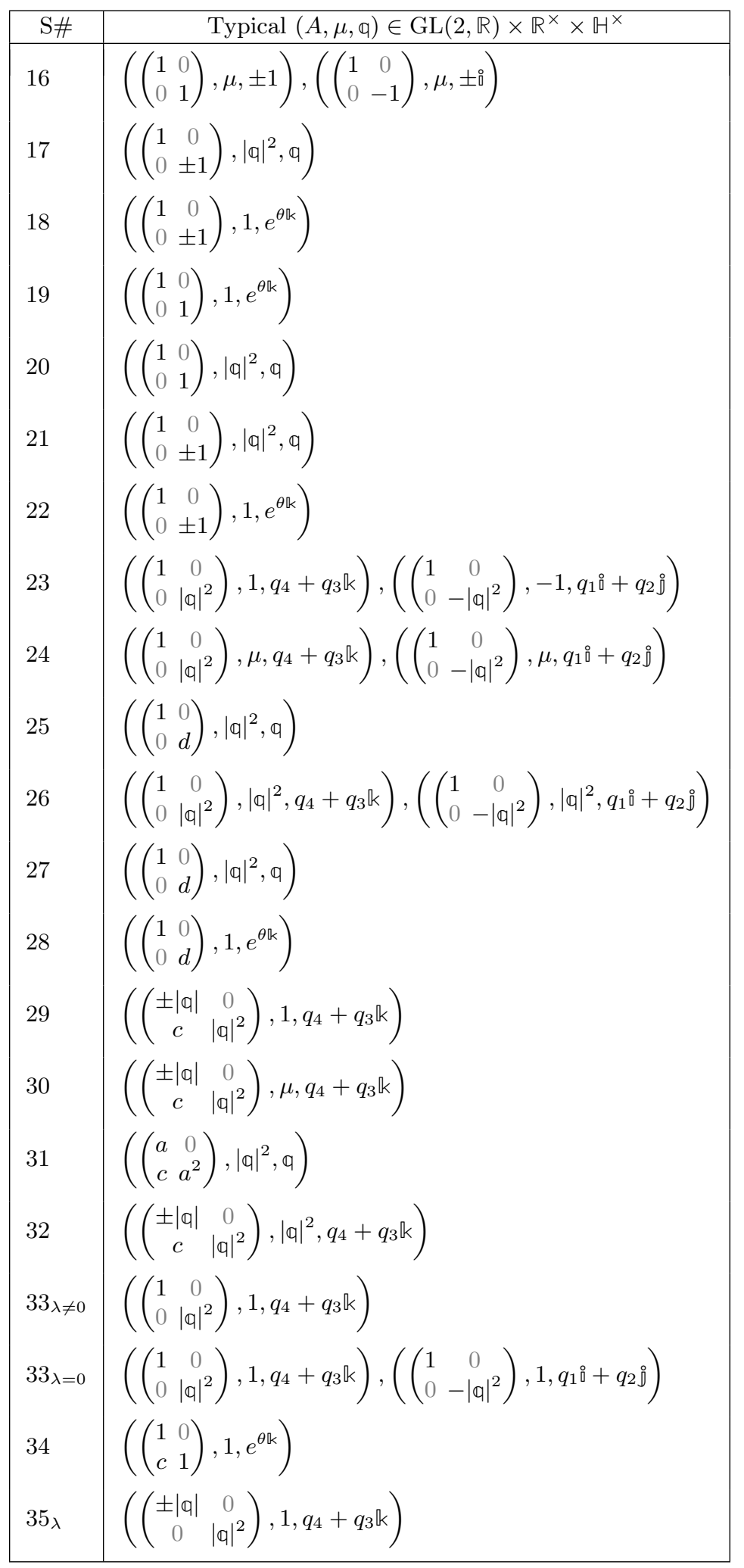

Table 9. Automorphisms of kinematical Lie superalgebras (continued). 
All the homogeneous supermanifolds in this paper are split: $\mathcal{O}$ is isomorphic to the sheaf of sections of the exterior algebra bundle of a homogeneous vector bundle $E \rightarrow M$; that is,

$$
\mathcal{O}(U)=\Gamma\left(U, \oplus_{p \geq 0} \wedge^{p} E\right) \quad \text { with } \quad \mathcal{N}(U)=\Gamma\left(U, \oplus_{p \geq 1} \wedge^{p} E\right) .
$$

A celebrated theorem of Batchelor's states that any smooth supermanifold always admits a splitting; although the splitting is not canonical [20].

Lie supergroups can be described as group objects in the category of supermanifolds, but there is an equivalent description in terms of Harish-Chandra pairs. Indeed, there is an equivalence of categories between Lie supergroups and Harish-Chandra pairs [19, 21] $(\mathcal{K}, \mathfrak{s})$ consisting of a Lie group $\mathcal{K}$ and a Lie superalgebra $\mathfrak{s}=\mathfrak{s}_{\overline{0}} \oplus \mathfrak{s}_{\overline{1}}$ where the Lie algebra of $\mathcal{K}$ is (isomorphic to) $\mathfrak{s}_{\overline{0}}$ and where the adjoint action of $\mathfrak{s}_{\overline{0}}$ on $\mathfrak{s}$ lifts to an action of $\mathcal{K}$ on $\mathfrak{s}$ by automorphisms. By a result of Koszul [21] (see also [18], Thm. 2.2) the structure sheaf of the Lie supergroup corresponding to a Harish-Chandra pair $(\mathcal{K}, \mathfrak{s})$ is the sheaf of smooth functions $\mathcal{K} \rightarrow \wedge^{\bullet} \mathfrak{s}_{\overline{1}}$, which can be interpreted as the sheaf of smooth sections of the trivial vector bundle $\mathcal{K} \times \wedge^{\bullet} \mathfrak{s}_{\overline{1}}$ over $\mathcal{K}$.

Now suppose that $M$ is a simply-connected homogeneous manifold realising a pair $(\mathfrak{k}, \mathfrak{h})$. Recall that this means that $M=\mathcal{K} / \mathcal{H}$ where $\mathcal{K}$ is a connected and simply-connected Lie group with Lie algebra $\mathfrak{k}$ and $\mathcal{H}$ is the connected Lie subgroup of $\mathcal{K}$ generated by $\mathfrak{h}$, assumed closed. Suppose that $\mathfrak{s}=\mathfrak{s}_{\overline{0}} \oplus \mathfrak{s}_{\overline{1}}$ is a Lie superalgebra with $\mathfrak{s}_{\overline{0}}=\mathfrak{k}$. Then $S:=\mathfrak{s}_{\overline{1}}$ is a representation of $\mathfrak{k}$ and, since $\mathcal{K}$ is simply-connected, it is also a representation of $\mathcal{K}$ and, by restriction, also a representation of $\mathcal{H}$. Let $E:=\mathcal{K} \times_{\mathcal{H}} S$ denote the homogeneous vector bundle over $M$ associated with the representation $S$ of $\mathcal{H}$. We define a supermanifold $(M, \mathcal{O})$ where $\mathcal{O}$ is the sheaf of sections of the exterior bundle $\wedge^{\bullet} E$. This supermanifold is called the superisation of $M$ defined by the Lie superalgebra $\mathfrak{s}$ (cf. [18], Thm. 5.6).

Conversely, any homogeneous supermanifold is of this form. Although the result is more general, we need only the special case where $\mathcal{H} \subset \mathcal{K}$ is a closed Lie subgroup. Then the homogeneous superisation of $\mathcal{K} / \mathcal{H}$ has as structure sheaf the $\mathcal{H}$-equivariant smooth functions $\mathcal{K} \rightarrow \wedge^{\bullet} \mathfrak{s}_{\overline{1}}$ (cf. [18], subsection 3.3), but these are precisely the smooth sections of the homogeneous vector bundle over $\mathcal{K} / \mathcal{H}$ associated to the representation $\wedge^{\mathfrak{s}_{\overline{1}}}$ of $\mathcal{H}$.

Therefore to every homogeneous superisation of $\mathcal{K} / \mathcal{H}$ we may associate a pair $(\mathfrak{s}, \mathfrak{h})$ and, conversely, every pair $(\mathfrak{s}, \mathfrak{h})$ defines a homogeneous superisation of $\mathcal{K} / \mathcal{H}$. Let us formally define these pairs in our present context.

Definition 3. A super Lie pair consists of a pair $(\mathfrak{s}, \mathfrak{h})$ where $\mathfrak{s}$ is one of the kinematical Lie superalgebras in table 4 and $\mathfrak{h}$ is a Lie subalgebra containing $\mathfrak{r}$ and decomposing as $\mathfrak{h}=\mathfrak{r} \oplus V$ under the adjoint action of $\mathfrak{r}$, where $V \subset \mathfrak{s}_{\overline{0}}$ is a copy of the vector representation. Just as in the non-super case discussed in [16], we shall refer to such Lie subalgebras as admissible. Two super Lie pairs $(\mathfrak{s}, \mathfrak{h})$ and $\left(\mathfrak{s}, \mathfrak{h}^{\prime}\right)$ are isomorphic if there is an automorphism of $\mathfrak{s}$ under which $\mathfrak{h}$ goes to $\mathfrak{h}^{\prime}$. We shall say that a super Lie pair $(\mathfrak{s}, \mathfrak{h})$ is geometrically realisable if and only if so is the Lie pair $(\mathfrak{k}, \mathfrak{h})$, where $\mathfrak{k}=\mathfrak{s}_{\overline{0}}$. We say that a super Lie pair $(\mathfrak{s}, \mathfrak{h})$ is effective if $\mathfrak{h}$ does not contain an ideal of $\mathfrak{s}$.

We observe that the condition of being geometrically realisable has nothing to do with supersymmetry, whereas the condition of being effective does take into account the 
whole superalgebra. It is thus possible, and indeed we will see examples below, that a geometrically realisable super Lie pair $(\mathfrak{s}, \mathfrak{h})$ is effective, but the underlying pair $(\mathfrak{k}, \mathfrak{h})$ is not. In that case, the vectorial generators in $\mathfrak{h}$ act trivially on the body of the superspace, but nontrivially on the fermionic coordinates; that is, they generate R-symmetries.

As in the classical theory, there is a one-to-one correspondence between (isomorphism classes of) effective, geometrically realisable super Lie pairs and (isomorphism classes of) homogeneous superisations of homogeneous manifolds. To the best of our knowledge, this result is part of the mathematical folklore and we are not aware of any reference where this result is proved or even stated as such.

\subsection{Admissible super Lie pairs}

We are now ready to classify admissible super Lie pairs up to isomorphism. We recall these are pairs $(\mathfrak{s}, \mathfrak{h})$, where $\mathfrak{s}$ is one of the kinematical Lie superalgebras in table 4 and $\mathfrak{h}$ is a Lie subalgebra $\mathfrak{h} \subset \mathfrak{k}=\mathfrak{s}_{0}$ which is admissible in the sense of [4]; that is, it contains the rotational subalgebra $\mathfrak{r}$ and, as a representation of $\mathfrak{r}, \mathfrak{h}=\mathfrak{r} \oplus V$ where $V \subset \mathfrak{k}$ is a copy of the vector representation. Two super Lie pairs $(\mathfrak{s}, \mathfrak{h})$ and $\left(\mathfrak{s}, \mathfrak{h}^{\prime}\right)$ are isomorphic if there is an automorphism of $\mathfrak{s}$ which maps $\mathfrak{h}$ (isomorphically) to $\mathfrak{h}^{\prime}$. As in ([4], section 3 ), our strategy in classifying admissible super Lie pairs up to isomorphism will be to take each kinematical Lie superalgebra $\mathfrak{s}$ in table 4 in turn, determine the admissible subalgebras $\mathfrak{h}$ and study the action of the automorphisms in tables 8 and 9 on the space of admissible subalgebras in order to select one representative from each orbit. In particular, every admissible super Lie pair $(\mathfrak{s}, \mathfrak{h})$ defines a unique admissible Lie pair $(\mathfrak{k}, \mathfrak{h})$ which, if effective and geometrically realisable, is associated with a unique simply-connected kinematical homogeneous spacetime $\mathcal{K} / \mathcal{H}$. That being the case, we may think of the super Lie pair $(\mathfrak{s}, \mathfrak{h})$ as a homogeneous kinematical superspacetime which superises $\mathcal{K} / \mathcal{H}$.

Without loss of generality - since an admissible subalgebra $\mathfrak{h}$ contains $\mathfrak{r}$ - the vectorial complement $V$ can be taken to be the span of $\alpha B_{i}+\beta P_{i}, i=1,2,3$, for some $\alpha, \beta \in \mathbb{R}$ not both zero, since the spans of $\left\{J_{i}, \alpha B_{i}+\beta P_{i}\right\}$ and of $\left\{J_{i}, \alpha B_{i}+\beta P_{i}+\gamma J_{i}\right\}$ coincide for all $\gamma \in \mathbb{R}$. We will often use the shorthand $V=\alpha \boldsymbol{B}+\beta \boldsymbol{P}$. The determination of the possible admissible subalgebras can be found in ([4], subsections 3.1-2), but we cannot simply import the results of that paper wholesale because here we are only allowed to act with automorphisms of $\mathfrak{s}$ and not just of $\mathfrak{k}$.

As in that paper, we will eventually change basis in the Lie superalgebra $\mathfrak{s}$ so that the admissible subalgebra $\mathfrak{h}$ is spanned by $\boldsymbol{J}$ and $\boldsymbol{B}$. Hence in determining the possible super Lie pairs, we will keep track of the required change of basis, ensuring, where possible, that $(\mathfrak{s}, \mathfrak{h})$ is reductive; that is, such that $H, P_{i}, Q_{a}$ (defined by equation (2.4)) span a subspace $\mathfrak{m} \subset \mathfrak{s}$ complementary to $\mathfrak{h}$ and such that $[\mathfrak{h}, \mathfrak{m}] \subset \mathfrak{m}$. This is equivalent to requiring that the span $\mathfrak{m}_{\overline{0}}$ of $H, P_{i}$ satisfies $\left[\mathfrak{h}, \mathfrak{m}_{\overline{0}}\right] \subset \mathfrak{m}_{\overline{0}}$, since the $Q_{i}$ span $\mathfrak{s}_{\overline{1}}$ and $\left[\mathfrak{h}, \mathfrak{s}_{\overline{1}}\right] \subset \mathfrak{s}_{\overline{1}}$ by virtue of $\mathfrak{s}$ being a Lie superalgebra.

It follows by inspection of ([4], subsections $3.1-2)$ that the Lie superalgebras $\mathfrak{s}$ whose automorphisms are listed in table 8 are extensions of kinematical Lie algebras $\mathfrak{k}$ for which any vectorial subspace $V=\alpha \boldsymbol{B}+\beta \boldsymbol{P}$ defines an admissible subalgebra $\mathfrak{h}=\mathfrak{r} \oplus V \subset \mathfrak{k}$. It is then a simple matter to determine the orbits of the action of the automorphisms 
listed in table 8 on the space of vectorial subspaces and hence to arrive at a list of possible inequivalent super Lie pairs $(\mathfrak{s}, \mathfrak{h})$ for such $\mathfrak{s}$.

It also follows by inspection of [4], subsections 3.1-2) that, of the remaining Lie superalgebras (i.e., those whose automorphisms are listed in table 9), most are extensions of kinematical Lie algebras possessing a unique vectorial subspace $V$ for which $\mathfrak{h}=\mathfrak{r} \oplus V$ is an admissible subalgebra. The exceptions are those Lie superalgebras S23-S28 and S33 which are extensions of the kinematical Lie algebras K14 and K16, respectively, for which there are precisely two vectorial subspaces leading to admissible subalgebras.

Let us concentrate first on the Lie superalgebras S1-S15, whose automorphisms are listed in table 8. As mentioned above, for $V$ any vectorial subspace, $\mathfrak{h}=\mathfrak{r} \oplus V$ is an admissible subalgebra. We need to determine the orbits of the action of the automorphisms in table 8. Since $V=\alpha \boldsymbol{B}+\beta \boldsymbol{P}$, this is equivalent to studying the action of the matrix part $A$ of the automorphism $(A, \mu, q)$ on nonzero vectors $(\alpha, \beta) \in \mathbb{R}^{2}$. In fact, since $(\alpha, \beta)$ and $(\lambda \alpha, \lambda \beta)$ for $0 \neq \lambda \in \mathbb{R}$ denote the same vectorial subspace, we must study the action of the subgroup of $\mathrm{GL}(2, \mathbb{R})$ defined by the matrices $A$ in the automorphism group on the projective space $\mathbb{R P}^{1}$. The map $(A, \mu, \mathfrak{q}) \mapsto A$ defines a group homomorphism from the automorphism group of a Lie superalgebra $\mathfrak{s}$ to $\operatorname{GL}(2, \mathbb{R})$. We will let $\mathcal{A}$ denote the image of this homomorphism: it is a subgroup of $\operatorname{GL}(2, \mathbb{R})$ and it is the action of $\mathcal{A}$ on $\mathbb{R P}^{1}$ that we need to investigate. Of course, $\mathcal{A}$ depends on $\mathfrak{s}$, even though we choose not to overload the notation by making this dependence explicit.

It follows by inspection of table 8 , that for $\mathfrak{s}$ any of the Lie superalgebras $\mathrm{S} 2, \mathrm{~S} 4, \mathrm{~S} 5$, $\mathrm{S} 11_{\chi \geq 0}, \mathrm{~S} 13$ and $\mathrm{S} 15$, the subgroup $\mathcal{A} \subset \mathrm{GL}(2, \mathbb{R})$ acts transitively on $\mathbb{R P}^{1}$ and hence for such Lie superalgebras there is a unique admissible subalgebra spanned by $\boldsymbol{J}$ and $\boldsymbol{B}$.

In contrast, if $\mathfrak{s}$ is any of the Lie superalgebras S1, S3, S6, S7, S8, S9 $9_{\gamma=1, \lambda \in \mathbb{R}}, \mathrm{S} 12_{\lambda \in \mathbb{R}}$ and S14, the subgroup $\mathcal{A} \subset \mathrm{GL}(2, \mathbb{R})$ acts with two orbits on $\mathbb{R P}^{1}$. For example, consider the Lie superalgebra $\mathrm{S} 1$, for which any $A \in \mathcal{A}$ takes the form

$$
\left(\begin{array}{ll}
a & 0 \\
c & d
\end{array}\right) \quad \text { for some } a, c, d \in \mathbb{R} \text { with } a, d \neq 0,
$$

and act as

$$
\left(\begin{array}{l}
\alpha \\
\beta
\end{array}\right) \mapsto\left(\begin{array}{ll}
a & 0 \\
c & d
\end{array}\right)\left(\begin{array}{l}
\alpha \\
\beta
\end{array}\right)=\left(\begin{array}{c}
a \alpha \\
d \beta+c \alpha
\end{array}\right) .
$$

If $\alpha \neq 0$, we can choose $c=-d \beta / \alpha$ to bring $(\alpha, \beta)$ to $(a \alpha, 0)$ which is projectively equivalent to $(1,0)$. On the other hand, if $\alpha=0$, then we cannot change that via automorphisms and hence we have $(0, \beta)$, which is projectively equivalent to $(0,1)$. In summary, we have two inequivalent admissible subalgebras with vectorial subspaces $V=\boldsymbol{B}$ and $V=\boldsymbol{P}$. The same result holds for the other Lie superalgebras in this list.

For the cases where $V=\boldsymbol{P}$ we change basis in the Lie superalgebra $\mathfrak{s}$ so that the admissible subalgebra $\mathfrak{h}$ is spanned by $\boldsymbol{J}$ and $\boldsymbol{B}$. This results in different brackets, which we now proceed to list.

Finally, if $\mathfrak{s}$ is any of the Lie superalgebras $S 9_{\gamma \neq 1, \lambda \in \mathbb{R}}$ and $S 10_{\gamma, \lambda \in \mathbb{R}}$, the subgroup $\mathcal{A} \subset \mathrm{GL}(2, \mathbb{R})$ acts with three orbits. Indeed, the matrices $A \in \mathcal{A}$ are now diagonal and of 


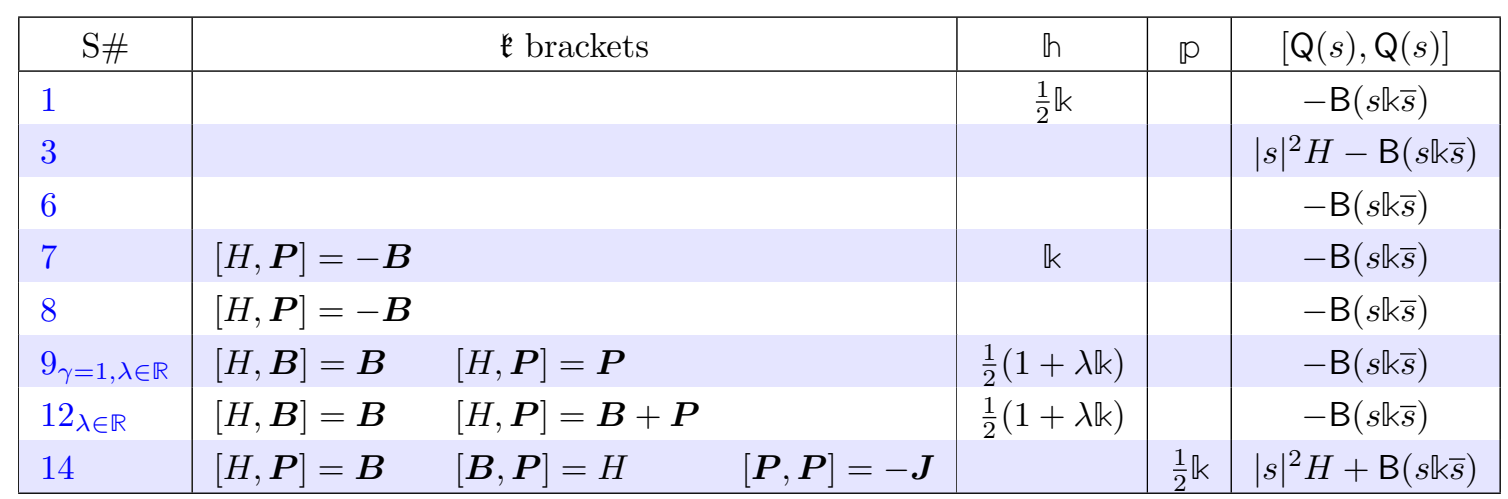

Table 10. Super Lie pairs (with $V=\boldsymbol{P}$ ).

the form

$$
\left(\begin{array}{ll}
a & 0 \\
0 & d
\end{array}\right),
$$

where at least one of $a, d$ can take any nonzero value. If $(\alpha, \beta)$ is such that $\alpha=0$ or $\beta=0$, we cannot alter this via automorphisms and hence projectively we have either $(1,0)$ or $(0,1)$. If $\alpha \beta \neq 0$, then we can always bring it to $(1,1)$ or $(-1,-1)$ via an automorphism, but these are projectively equivalent. In summary, we have three orbits, corresponding to $V=\boldsymbol{B}, V=\boldsymbol{P}$ and $V=\boldsymbol{B}+\boldsymbol{P}$.

When $V=\boldsymbol{P}$, the Lie brackets of $S 9_{\gamma \neq 1, \lambda \in \mathbb{R}}$ in the new basis are given by

$[H, \boldsymbol{B}]=\boldsymbol{B}, \quad[H, \boldsymbol{P}]=\gamma \boldsymbol{P}, \quad[H, \mathrm{Q}(s)]=\mathrm{Q}\left(\frac{1}{2} s(1+\lambda \mathbb{k})\right) \quad$ and $\quad[\mathrm{Q}(s), \mathrm{Q}(s)]=-\mathrm{B}(s \mathbb{k} \bar{s})$,

and those of $S 10_{\gamma, \lambda \in \mathbb{R}}$ by

$[H, \boldsymbol{B}]=\boldsymbol{B}, \quad[H, \boldsymbol{P}]=\gamma \boldsymbol{P}, \quad[H, \mathrm{Q}(s)]=\mathrm{Q}\left(\frac{1}{2} s(\gamma+\lambda \mathbb{k})\right) \quad$ and $\quad[\mathrm{Q}(s), \mathrm{Q}(s)]=-\mathrm{P}(s \mathbb{k} \bar{s})$.

On the other hand, when $V=\boldsymbol{B}+\boldsymbol{P}$, the Lie brackets of $\mathrm{S} 9_{\gamma \neq 1, \lambda \in \mathbb{R}}$ in the new basis are given by

$$
\begin{aligned}
& {[H, \boldsymbol{B}]=-\boldsymbol{P}} \\
& {[H, \boldsymbol{P}]=\gamma \boldsymbol{B}+(1+\gamma) \boldsymbol{P}} \\
& {[H, \mathrm{Q}(s)]=\mathrm{Q}\left(\frac{1}{2} s(1+\lambda \mathbb{k})\right)} \\
& {[\mathrm{Q}(s), \mathrm{Q}(s)]=\frac{1}{1-\gamma}(\gamma \mathrm{B}(s \mathbb{k} \bar{s})+\mathrm{P}(s \mathbb{k} \bar{s})) \text {, }}
\end{aligned}
$$

and those of $\mathrm{S}_{10}, \lambda \in \mathbb{R}$ by

$$
\begin{aligned}
{[H, \boldsymbol{B}] } & =-\boldsymbol{P} & {[H, \mathrm{Q}(s)] } & =\mathrm{Q}\left(\frac{1}{2} s(\gamma+\lambda \mathbb{k})\right) \\
{[H, \boldsymbol{P}] } & =\gamma \boldsymbol{B}+(1+\gamma) \boldsymbol{P} & {[\mathrm{Q}(s), \mathrm{Q}(s)] } & =\frac{1}{\gamma-1}(\mathrm{~B}(s \mathbb{k} \bar{s})+\mathrm{P}(s \mathbb{k} \bar{s})) .
\end{aligned}
$$

Now we turn to the Lie superalgebras whose automorphisms are listed in table 9 . If $\mathfrak{s}$ is one such Lie superalgebra, not every vectorial subspace leads to an admissible subalgebra. From the results in ([4], subsections 3.1-2) we have that Lie superalgebras S16-S22 admit a unique admissible subalgebra with $V=\boldsymbol{B}$, whereas for the Lie superalgebras S29-S32, S34 and $\mathbf{S} 35_{\lambda \in \mathbb{R}}$ also admit a unique admissible subalgebra with $V=\boldsymbol{P}$. Finally, the Lie 


\begin{tabular}{|c|c|c|c|c|c|}
\hline $\mathrm{S} \#$ & & $\mathfrak{k}$ brackets & Th & p & {$[\mathrm{Q}(s), \mathrm{Q}(s)]$} \\
\hline 23 & {$[\boldsymbol{P}, \boldsymbol{P}]=\boldsymbol{P}$} & & $\mathbb{k}$ & & $-\mathrm{B}(s \mathbb{k} \bar{s})$ \\
\hline 24 & {$[\boldsymbol{P}, \boldsymbol{P}]=\boldsymbol{P}$} & & & & $-\mathrm{B}(s \mathbb{k} \bar{s})$ \\
\hline 25 & {$[\boldsymbol{P}, \boldsymbol{P}]=\boldsymbol{P}$} & & & & $|s|^{2} H$ \\
\hline 26 & {$[\boldsymbol{P}, \boldsymbol{P}]=\boldsymbol{P}$} & & & & $|s|^{2} H-\mathrm{B}(s \llbracket \bar{s})$ \\
\hline 27 & {$[\boldsymbol{P}, \boldsymbol{P}]=\boldsymbol{P}$} & & & $\frac{1}{2}$ & $|s|^{2} H$ \\
\hline 28 & {$[\boldsymbol{P}, \boldsymbol{P}]=\boldsymbol{P}$} & & $\frac{1}{2} \mathbb{k}$ & $\frac{1}{2}$ & $|s|^{2} H-\mathrm{P}(s \llbracket \bar{s})$ \\
\hline 29 & {$[\boldsymbol{P}, \boldsymbol{P}]=\boldsymbol{B}$} & & $\mathbb{k}$ & & $-\mathrm{B}(s \mathbb{k} \bar{s})$ \\
\hline 30 & {$[\boldsymbol{P}, \boldsymbol{P}]=\boldsymbol{B}$} & & & & $-\mathrm{B}(s \mathbb{k} \bar{s})$ \\
\hline 31 & {$[\boldsymbol{P}, \boldsymbol{P}]=\boldsymbol{B}$} & & & & $|s|^{2} H$ \\
\hline 32 & {$[\boldsymbol{P}, \boldsymbol{P}]=\boldsymbol{B}$} & & & & 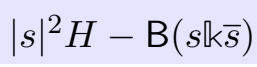 \\
\hline $33_{\lambda \in \mathbb{R}}$ & {$[H, \boldsymbol{B}]=\boldsymbol{B}$} & {$[\boldsymbol{P}, \boldsymbol{P}]=\boldsymbol{P}$} & $\frac{1}{2}(1+\lambda \mathbb{k})$ & & $-\mathrm{B}(s \mathbb{k} \bar{s})$ \\
\hline 34 & {$[H, \boldsymbol{P}]=-\boldsymbol{B}$} & {$[\boldsymbol{P}, \boldsymbol{P}]=\boldsymbol{B}$} & $\frac{1}{2} \mathbb{k}$ & & $-\mathrm{B}(s \llbracket \bar{s})$ \\
\hline $35_{\lambda \in \mathbb{R}}$ & {$[H, \boldsymbol{P}]=\boldsymbol{P}$} & {$[H, \boldsymbol{B}]=2 \boldsymbol{B} \quad[\boldsymbol{P}, \boldsymbol{P}]=\boldsymbol{B}$} & $1+\lambda \mathbb{k}$ & & $-\mathrm{B}(s \mathbb{k} \bar{s})$ \\
\hline
\end{tabular}

Table 11. More super Lie pairs (with $V=\boldsymbol{P}$ ).

superalgebras S23-S28 and S33 ${ }_{\lambda \in \mathbb{R}}$ admit precisely two admissible subalgebras with $V=\boldsymbol{B}$ and $V=\boldsymbol{P}$, which cannot be related by automorphisms.

Table 12 summarises the above results. For each Lie superalgebra $\mathfrak{s}$ in table 4 it lists the admissible subalgebras $\mathfrak{h}$ and hence the possible super Lie pairs $(\mathfrak{s}, \mathfrak{h})$. The notation for $\mathfrak{h}$ is simply the generators of the vectorial subspace $V \subset \mathfrak{h}$, where the span of $\alpha B_{a}+\beta P_{a}$ is abbreviated as $\alpha \boldsymbol{B}+\beta \boldsymbol{P}$. The blue entries correspond to effective super Lie pairs, whereas the green and greyed out correspond to non-effective super Lie pairs: the green ones giving rise to aristotelian superspaces upon quotienting by ideal. In section 3.2, we classified aristotelian Lie superspaces by classifying their corresponding aristotelian Lie superalgebras (see table 6 ) and in section 4.4 we exhibit the precise correspondence between the aristotelian non-effective super Lie pairs and the aristotelian superspaces (see table 13).

\subsection{Effective super Lie pairs}

Recall that a super Lie pair $(\mathfrak{s}, \mathfrak{h})$ is said to be effective if $\mathfrak{h}$ does not contain an ideal of $\mathfrak{s}$. Since $\mathfrak{h} \subset \mathfrak{k}$ and contains the rotational subalgebra, which has nonvanishing brackets with $\boldsymbol{Q}$, the only possible ideal of $\mathfrak{s}$ contained in $\mathfrak{h}$ would be the vectorial subspace $V \subset \mathfrak{h}$. It is then a simple matter to inspect the super Lie pairs determined in the previous section and select those for which $V$ is not an ideal of $\mathfrak{s}$. Those super Lie pairs have been highlighted in blue in table 12 . We now take each such super Lie pair in turn, change basis if needed so that $V$ is spanned by $\boldsymbol{B}$, and then list the resulting brackets in that basis. Every such super Lie pair $(\mathfrak{s}, \mathfrak{h})$ determines a Lie pair $(\mathfrak{k}, \mathfrak{h})$. If the Lie pair $(\mathfrak{k}, \mathfrak{h})$ is effective (and geometrically realisable), then $(\mathfrak{s}, \mathfrak{h})$ describes a homogeneous superisation of one of 


\begin{tabular}{|c|c|c|c|c|}
\hline $\mathfrak{s}$ & $\mathfrak{k}$ & \multicolumn{3}{|c|}{$V \subset \mathfrak{h}$} \\
\hline S1 & K1 & $B$ & $P$ & \\
\hline S2 & K1 & $B$ & & \\
\hline S3 & K1 & $B$ & $P$ & \\
\hline S4 & K1 & $B$ & & \\
\hline S5 & K1 & $B$ & & \\
\hline S6 & $\mathrm{K} 1$ & $B$ & $P$ & \\
\hline S7 & $\mathrm{K} 2$ & $B$ & $P$ & \\
\hline S8 & $\mathrm{K} 2$ & $B$ & $P$ & \\
\hline $\mathrm{S} 9_{\gamma \in[-1,1), \lambda \in \mathbb{R}}$ & $\mathrm{K} 3_{\gamma}$ & $B$ & $P$ & $B+P$ \\
\hline $\mathrm{S} 9_{\gamma=1, \lambda \in \mathbb{R}}$ & $\mathrm{K} 3_{\gamma=1}$ & $B$ & $P$ & \\
\hline $\mathrm{S}^{10}{ }_{\gamma \in[-1,1), \lambda \in \mathbb{R}}$ & $\mathrm{K} 3_{\gamma}$ & $B$ & $P$ & $B+P$ \\
\hline $\mathrm{S} 11_{\chi \geq 0}$ & $\mathrm{~K} 4_{\chi}$ & $B$ & & \\
\hline
\end{tabular}

\begin{tabular}{|l|l|l|l|}
\hline \multicolumn{1}{|c|}{$\mathfrak{s}$} & \multicolumn{1}{c|}{$\mathfrak{k}$} & \multicolumn{2}{|c|}{$V \subset \mathfrak{h}$} \\
\hline $\mathrm{S} 12_{\lambda \in \mathbb{R}}$ & $\mathrm{K} 5$ & $\boldsymbol{B}$ & $P$ \\
$\mathrm{~S} 13$ & $\mathrm{~K} 6$ & $\boldsymbol{B}$ & \\
$\mathrm{S} 14$ & $\mathrm{~K} 8$ & $\boldsymbol{B}$ & $\boldsymbol{P}$ \\
$\mathrm{S} 15$ & $\mathrm{~K} 11$ & $\boldsymbol{B}$ & \\
$\mathrm{S} 16$ & $\mathrm{~K} 12$ & $\boldsymbol{B}$ & \\
$\mathrm{S} 17$ & $\mathrm{~K} 12$ & $\boldsymbol{B}$ & \\
$\mathrm{S} 18$ & $\mathrm{~K} 12$ & $\boldsymbol{B}$ & \\
$\mathrm{S} 19$ & $\mathrm{~K} 13$ & $\boldsymbol{B}$ & \\
$\mathrm{S} 20$ & $\mathrm{~K} 13$ & $\boldsymbol{B}$ & \\
$\mathrm{S} 21$ & $\mathrm{~K} 13$ & $\boldsymbol{B}$ & \\
$\mathrm{S} 22$ & $\mathrm{~K} 13$ & $\boldsymbol{B}$ & \\
$\mathrm{S} 23$ & $\mathrm{~K} 14$ & $\boldsymbol{B}$ & $P$ \\
\hline
\end{tabular}

\begin{tabular}{|l|c|c|c|}
\hline \multicolumn{1}{|c|}{$\mathfrak{s}$} & $\mathfrak{k}$ & \multicolumn{2}{|c|}{$V \subset \mathfrak{h}$} \\
\hline S24 & $\mathrm{K} 14$ & $\boldsymbol{B}$ & $P$ \\
S25 & $\mathrm{K} 14$ & $\boldsymbol{B}$ & $\boldsymbol{P}$ \\
S26 & $\mathrm{K} 14$ & $\boldsymbol{B}$ & $\boldsymbol{P}$ \\
S27 & $\mathrm{K} 14$ & $\boldsymbol{B}$ & $\boldsymbol{P}$ \\
S28 & $\mathrm{K} 14$ & $\boldsymbol{B}$ & $\boldsymbol{P}$ \\
S29 & $\mathrm{K} 15$ & $P$ & \\
S30 & $\mathrm{K} 15$ & $P$ & \\
S31 & $\mathrm{K} 15$ & $\boldsymbol{P}$ & \\
S32 & $\mathrm{K} 15$ & $\boldsymbol{P}$ & \\
S33 & $\mathrm{K} 16$ & $\boldsymbol{B}$ & $P$ \\
S34 & $\mathrm{K} 17$ & $P$ & \\
S35 $_{\lambda \in \mathbb{R}}$ & $\mathrm{K} 18$ & $P$ & \\
\hline
\end{tabular}

Table 12. Summary of super Lie pairs.

The blue pairs (e.g., $\boldsymbol{B}$ ) are effective; the green pairs (e.g., $\boldsymbol{B}$ ) though not effective, give rise to aristotelian superspaces; whereas the greyed out pairs (e.g., B) are not effective and will not be considered further.

the spatially-isotropic homogeneous spacetimes in [4]. We remark that there are effective super Lie pairs $(\mathfrak{s}, \mathfrak{h})$ for which the underlying Lie pair $(\mathfrak{k}, \mathfrak{h})$ is not effective. In those cases, there are no boosts on the body of the superspacetime, but instead there are R-symmetries in the odd coordinates.

As usual, in writing the Lie brackets of $\mathfrak{s}$ below we do not include any bracket involving $\boldsymbol{J}$, which are given in equation (2.5) and instead give any non-zero additional brackets.

\subsubsection{Galilean superspaces}

Galilean spacetime is described by $(\mathfrak{k}, \mathfrak{h})$ where $\mathfrak{k}$ has the additional bracket $[H, \boldsymbol{B}]=-\boldsymbol{P}$. There are two possible superisations $(\mathfrak{s}, \mathfrak{h})$, with brackets

$$
[H, \mathrm{Q}(s)]=\left\{\begin{array}{l}
\mathrm{Q}(s \mathbb{k}) \\
0
\end{array} \quad \text { and } \quad[\mathrm{Q}(s), \mathrm{Q}(s)]=-\mathrm{P}(s \mathbb{k} \bar{s})\right.
$$

These are associated with Lie superalgebras S7 and S8 in table 4.

\subsubsection{Galilean de Sitter superspace}

Galilean de Sitter spacetime is described by $(\mathfrak{k}, \mathfrak{h})$ where $\mathfrak{k}$ has the additional brackets $[H, \boldsymbol{B}]=-\boldsymbol{P}$ and $[H, \boldsymbol{P}]=-\boldsymbol{B}$. There are two one-parameter family of superisations $(\mathfrak{s}, \mathfrak{h})$, with brackets

$$
[H, \mathrm{Q}(s)]=\mathrm{Q}\left(\frac{1}{2} s( \pm 1+\lambda \mathbb{k})\right) \quad \text { and } \quad[\mathrm{Q}(s), \mathrm{Q}(s)]=-\frac{1}{2}(\mathrm{~B}(s \mathbb{k} \bar{s}) \mp \mathrm{P}(s \mathbb{k} \bar{s}))
$$

for $\lambda \in \mathbb{R}$. They are associated with Lie superalgebras $S 9_{\gamma=-1, \lambda}$ and $S 10_{\gamma=-1, \lambda}$, respectively. 


\subsubsection{Torsional galilean de Sitter superspaces}

Torsional galilean de Sitter spacetime is described by $(\mathfrak{k}, \mathfrak{h})$ where $\mathfrak{k}$ has the additional brackets $[H, \boldsymbol{B}]=-\boldsymbol{P}$ and $[H, \boldsymbol{P}]=\gamma \boldsymbol{B}+(1+\gamma) \boldsymbol{P}$, where $\gamma \in(-1,1)$. There are two one-parameter family of superisations $(\mathfrak{s}, \mathfrak{h})$, with brackets

$$
[H, \mathrm{Q}(s)]=\mathrm{Q}\left(\frac{1}{2} s(1+\lambda \mathbb{k})\right) \quad \text { and } \quad[\mathrm{Q}(s), \mathrm{Q}(s)]=\frac{1}{1-\gamma}(\gamma \mathrm{B}(s \mathbb{k} \bar{s})+\mathrm{P}(s \mathbb{k} \bar{s}))
$$

and

$$
[H, \mathrm{Q}(s)]=\mathrm{Q}\left(\frac{1}{2} s(\gamma+\lambda \mathbb{k})\right) \quad \text { and } \quad[\mathrm{Q}(s), \mathrm{Q}(s)]=\frac{1}{\gamma-1}(\mathrm{~B}(s \mathbb{k} \bar{s})+\mathrm{P}(s \mathbb{k} \bar{s}))
$$

for $\lambda \in \mathbb{R}$. The associated Lie superalgebras are $\mathrm{S} 9_{\gamma, \lambda}$ and $\mathrm{S} 10_{\gamma, \lambda}$, respectively.

For $\gamma=1$, with additional brackets $[H, \boldsymbol{B}]=-\boldsymbol{P}$ and $[H, \boldsymbol{P}]=\boldsymbol{B}+2 \boldsymbol{P}$, there is a one-parameter family of superisations, with brackets

$$
[H, \mathrm{Q}(s)]=\mathrm{Q}\left(\frac{1}{2} s(1+\lambda \mathbb{k})\right) \quad \text { and } \quad[\mathrm{Q}(s), \mathrm{Q}(s)]=\mathrm{B}(s \mathbb{k} \bar{s})+\mathrm{P}(s \mathbb{k} \bar{s}) .
$$

The associated Lie superalgebras are $\mathrm{S} 12_{\lambda}$.

\subsubsection{Galilean anti de Sitter superspace}

Galilean anti de Sitter spacetime is described by $(\mathfrak{k}, \mathfrak{h})$ where $\mathfrak{k}$ has the additional brackets $[H, \boldsymbol{B}]=-\boldsymbol{P}$ and $[H, \boldsymbol{P}]=\boldsymbol{B}$. It admits a superisation $(\mathfrak{s}, \mathfrak{h})$, with brackets

$$
[H, \mathrm{Q}(s)]=\mathrm{Q}\left(\frac{1}{2} s \stackrel{\jmath}{)} \quad \text { and } \quad[\mathrm{Q}(s), \mathrm{Q}(s)]=-\mathrm{B}(s \AA \bar{s})+\mathrm{P}(s \llbracket \bar{s}),\right.
$$

which corresponds to the Lie superalgebra $S 11_{\chi=0}$, after changing basis the sign of $\boldsymbol{P}$.

\subsubsection{Torsional galilean anti de Sitter superspace}

Torsional galilean anti de Sitter spacetime is described by $(\mathfrak{k}, \mathfrak{h})$ where $\mathfrak{k}$ has the additional brackets $[H, \boldsymbol{B}]=\chi \boldsymbol{B}+\boldsymbol{P}$ and $[H, \boldsymbol{P}]=\chi \boldsymbol{P}-\boldsymbol{B}$, where $\chi>0$. There is a unique superisation $(\mathfrak{s}, \mathfrak{h})$, with brackets

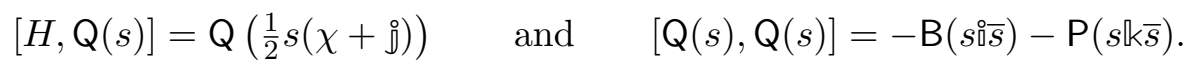

For uniformity, we change basis so that $[H, \boldsymbol{B}]=-\boldsymbol{P}$ as for all galilean spacetimes. Then the resulting super Lie pair $(\mathfrak{s}, \mathfrak{h})$ is determined by the brackets $[H, \boldsymbol{B}]=-\boldsymbol{P},[H, \boldsymbol{P}]=$ $\left(1+\chi^{2}\right) \boldsymbol{B}+2 \chi \boldsymbol{P}$ and, in addition,

$$
[H, \mathrm{Q}(s)]=\mathrm{Q}\left(\frac{1}{2} s(\chi+\AA)\right) \quad \text { and } \quad[\mathrm{Q}(s), \mathrm{Q}(s)]=\mathrm{B}(s \mathbb{k}(\chi+\jmath) \bar{s})+\mathrm{P}(s \mathbb{k} \bar{s}),
$$

corresponding to the Lie superalgebra $\mathrm{S} 11_{\chi}$.

\subsubsection{Carrollian superspace}

Carrollian spacetime is described by $(\mathfrak{k}, \mathfrak{h})$ where $\mathfrak{k}$ has the additional brackets $[\boldsymbol{B}, \boldsymbol{P}]=H$. It admits a superisation $(\mathfrak{s}, \mathfrak{h})$, with brackets

$$
[\mathrm{Q}(s), \mathrm{Q}(s)]=|s|^{2} H,
$$

which corresponds to the Lie superalgebra S13. 


\subsubsection{Minkowski superspace}

Minkowski superspace arises as a superisation of Minkowski spacetime, described by $(\mathfrak{k}, \mathfrak{h})$ with brackets $[H, \boldsymbol{B}]=-\boldsymbol{P},[\boldsymbol{B}, \boldsymbol{P}]=H$ and $[\boldsymbol{B}, \boldsymbol{B}]=-\boldsymbol{J}$ and in addition

$$
[\mathrm{B}(\beta), \mathrm{Q}(s)]=\mathrm{Q}\left(\frac{1}{2} \beta s \mathbb{k}\right) \quad \text { and } \quad[\mathrm{Q}(s), \mathrm{Q}(s)]=|s|^{2} H-\mathrm{P}(s \mathbb{k} \bar{s}) .
$$

This is, of course, the Poincaré superalgebra S14.

\subsubsection{Carrollian anti de Sitter superspace}

Carrollian anti de Sitter spacetime is described as $(\mathfrak{k}, \mathfrak{h})$ where the $\mathfrak{k}$ brackets are given by $[H, \boldsymbol{P}]=\boldsymbol{B},[\boldsymbol{B}, \boldsymbol{P}]=H$ and $[\boldsymbol{P}, \boldsymbol{P}]=-\boldsymbol{J}$. It admits a unique superisation $(\mathfrak{s}, \mathfrak{h})$ with brackets (we have rotated $\mathbb{k}$ to $\stackrel{\circ}{0}$ )

$$
[\mathrm{P}(\pi), \mathrm{Q}(s)]=\mathrm{Q}\left(\frac{1}{2} \pi s_{0}^{\circ}\right) \quad \text { and } \quad[\mathrm{Q}(s), \mathrm{Q}(s)]=|s|^{2} H+\mathrm{B}(s \circ \bar{s}) .
$$

We remark that just as with carrollian anti de Sitter and Minkowski spacetimes, which are both homogeneous spacetimes of the Poincaré group, their superisations have isomorphic supersymmetry algebras: namely, the Poincaré superalgebra S14.

\subsubsection{Anti de Sitter superspace}

Anti de Sitter spacetime is described kinematically as $(\mathfrak{k}, \mathfrak{h})$ with brackets

$$
[H, \boldsymbol{B}]=-\boldsymbol{P}, \quad[H, \boldsymbol{P}]=\boldsymbol{B}, \quad[\boldsymbol{B}, \boldsymbol{P}]=H, \quad[\boldsymbol{B}, \boldsymbol{B}]=-\boldsymbol{J} \quad \text { and } \quad[\boldsymbol{P}, \boldsymbol{P}]=-\boldsymbol{J} .
$$

It admits a unique superisation $(\mathfrak{s}, \mathfrak{h}$ ), with additional brackets (where we have rotated $(\stackrel{\circ}{i}, \mathfrak{j}, \mathbb{k}) \mapsto(\mathbb{k}, i, 0, j)$ for uniformity $)$

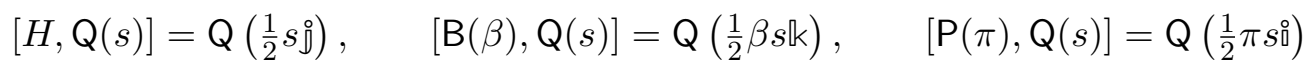

$$
\begin{aligned}
& \text { and } \quad[\mathrm{Q}(s), \mathrm{Q}(s)]=|s|^{2} H+\mathrm{J}(s \bar{\jmath} \bar{s})+\mathrm{B}(s \bar{\AA} \bar{s})-\mathrm{P}(s \llbracket \bar{s}) \text {. }
\end{aligned}
$$

The associated Lie superalgebra is $\mathrm{S} 15$, which is isomorphic to $\mathfrak{o s p}(1 \mid 4)$.

\subsubsection{Super-spacetimes extending $\mathbb{R} \times S^{3}$}

These correspond to the effective super Lie pairs associated with the Lie superalgebras S21 and S22. The super Lie pairs $(\mathfrak{s}, \mathfrak{h})$ are effective, but the underlying Lie pair $(\mathfrak{k}, \mathfrak{h})$ is not. Indeed, the brackets of $\mathfrak{k}$ are now $[\boldsymbol{B}, \boldsymbol{B}]=\boldsymbol{B}$ and $[\boldsymbol{P}, \boldsymbol{P}]=\boldsymbol{J}-\boldsymbol{B}$, from where we see that $\boldsymbol{B}$ spans an ideal of $\mathfrak{k}$; although not one of $\mathfrak{s}$, due to the brackets

$$
[\mathrm{B}(\beta), \mathrm{Q}(s)]=\mathrm{Q}\left(\frac{1}{2} \beta s\right) \quad \text { and } \quad[\mathrm{Q}(s), \mathrm{Q}(s)]=|s|^{2} H
$$

for $\mathfrak{s}$ the Lie superalgebra S21 or

$$
[H, \mathrm{Q}(s)]=\mathrm{Q}\left(\frac{1}{2} s \mathbb{k}\right), \quad[\mathrm{B}(\beta), \mathrm{Q}(s)]=\mathrm{Q}\left(\frac{1}{2} \beta s\right) \quad \text { and } \quad[\mathrm{Q}(s), \mathrm{Q}(s)]=|s|^{2} H-\mathrm{B}(s k \bar{s}),
$$

for $\mathfrak{s}$ the Lie superalgebra S22. In both superspaces, $\boldsymbol{B}$ do not generate boosts but Rsymmetries. The underlying spacetime in both cases is the Einstein static universe $\mathbb{R} \times S^{3}$. 


\subsubsection{Super-spacetimes extending $\mathbb{R} \times H^{3}$}

These correspond to the effective super Lie pairs associated with the Lie superalgebras S17 and S18. The super Lie pairs $(\mathfrak{s}, \mathfrak{h})$ are effective, but the underlying Lie pair $(\mathfrak{k}, \mathfrak{h})$ is not. Indeed, the brackets of $\mathfrak{k}$ are $[\boldsymbol{B}, \boldsymbol{B}]=\boldsymbol{B}$ and $[\boldsymbol{P}, \boldsymbol{P}]=\boldsymbol{B}-\boldsymbol{J}$, so that $\boldsymbol{B}$ span an ideal $\boldsymbol{v} \subset \mathfrak{k}$. The resulting aristotelian spacetime $(\mathfrak{k} / \boldsymbol{v}, \mathfrak{r})$ is the hyperbolic version of the Einstein static universe $\mathbb{R} \times H^{3}$.

For $\mathfrak{s}$ the Lie superalgebra S17, the brackets are

$$
[\mathrm{B}(\beta), \mathrm{Q}(s)]=\mathrm{Q}\left(\frac{1}{2} \beta s\right) \quad \text { and } \quad[\mathrm{Q}(s), \mathrm{Q}(s)]=|s|^{2} H,
$$

so that $\boldsymbol{B}$ does not span an ideal of $\mathfrak{s}$. In other words, $\boldsymbol{B}$ do not generate boosts in the underlying homogeneous spacetime, but rather R-symmetries.

A similar story holds for $\mathfrak{s}$ the Lie superalgebra $\mathbf{S 1 8}$, with the additional brackets

$$
[H, \mathrm{Q}(s)]=\mathrm{Q}\left(\frac{1}{2} s \mathbb{k}\right), \quad[\mathrm{B}(\beta), \mathrm{Q}(s)]=\mathrm{Q}\left(\frac{1}{2} \beta s\right) \quad \text { and } \quad[\mathrm{Q}(s), \mathrm{Q}(s)]=|s|^{2} H-\mathrm{B}(s \mathbb{k} \bar{s}) .
$$

Again, $\boldsymbol{B}$ are to be interpreted as R-symmetries.

\subsubsection{Super-spacetimes extending the static aristotelian spacetime}

This corresponds to the Lie superalgebras S27 and S28. In either case the resulting super Lie pair $(\mathfrak{s}, \mathfrak{h})$ is effective, but the underlying Lie pair $(\mathfrak{k}, \mathfrak{h})$ is not since $[\boldsymbol{B}, \boldsymbol{B}]=\boldsymbol{B}$ spans an ideal of $\mathfrak{k}$. The homogeneous spacetime associated with the non-effective $(\mathfrak{k}, \mathfrak{h})$ is the aristotelian static spacetime $\mathrm{S}$.

As in the previous cases, the generators $\boldsymbol{B}$ do not act as boosts but rather as Rsymmetries, as evinced by the brackets:

$$
[\mathrm{B}(\beta), \mathrm{Q}(s)]=\mathrm{Q}\left(\frac{1}{2} \beta s\right) \quad \text { and } \quad[\mathrm{Q}(s), \mathrm{Q}(s)]=|s|^{2} H .
$$

for $\mathfrak{s}$ the Lie superalgebra S27, or

$$
[H, \mathrm{Q}(s)]=\mathrm{Q}\left(\frac{1}{2} s \mathbb{k}\right), \quad[\mathrm{B}(\beta), \mathrm{Q}(s)]=\mathrm{Q}\left(\frac{1}{2} \beta s\right) \quad \text { and } \quad[\mathrm{Q}(s), \mathrm{Q}(s)]=|s|^{2} H-\mathrm{B}(s \llbracket \bar{s}) .
$$

for $\mathfrak{s}$ the Lie superalgebra S28.

\subsection{Aristotelian homogeneous superspaces}

The super Lie pairs $(\mathfrak{s}, \mathfrak{h})$ in green in table 12 are such that the vectorial subspace $V \subset \mathfrak{h}$ is an ideal $\boldsymbol{v}$ of $\mathfrak{s}$. Quotienting $\mathfrak{s}$ by this ideal yields a Lie superalgebra $\mathfrak{s a} \cong \mathfrak{s} / \boldsymbol{v}$ with $\mathfrak{a}=\mathfrak{s a}_{\overline{0}}$ an aristotelian Lie algebra (see [4], appendix A for a classification). The resulting aristotelian super Lie pair $(\mathfrak{s a}, \mathfrak{r})$ is effective by construction and geometrically realisable. It is then a simple matter to identify the aristotelian Lie superalgebra to which each of those non-effective super Lie pairs in table 12 leads. We summarise this in table 13, which exhibits the correspondence between aristotelian super Lie pairs in table 12 and aristotelian Lie superalgebras in table 6 . We identify the super Lie pair $(\mathfrak{s}, \mathfrak{h})$ by the label for $\mathfrak{s}$ as in table 4 and the ideal $\boldsymbol{v} \subset \mathfrak{h}$. 


\begin{tabular}{|l|l|l|}
\hline \multicolumn{1}{|c|}{$\mathfrak{s}$} & $\boldsymbol{v}$ & \multicolumn{1}{c|}{$\mathfrak{a}$} \\
\hline S1 & $\boldsymbol{B}$ & $\mathrm{S} 36$ \\
S2 & $\boldsymbol{B}$ & $\mathrm{S} 39$ \\
S3 & $\boldsymbol{B}$ & $\mathrm{S} 39$ \\
S3 & $\boldsymbol{P}$ & $\mathrm{S} 38$ \\
S4 & $\boldsymbol{B}$ & $\mathrm{S} 38$ \\
S5 & $\boldsymbol{B}$ & $\mathrm{S} 37$ \\
S6 & $\boldsymbol{B}$ & $\mathrm{S} 37$ \\
S9 $_{\gamma \in[-1,1), \lambda \in \mathbb{R}}$ & $\boldsymbol{B}$ & $\mathrm{S}_{\gamma_{\lambda}}$ \\
S9 $_{\gamma=1, \lambda \in \mathbb{R}}$ & $\boldsymbol{B}$ & $\mathrm{S}_{\lambda} 0_{\lambda}$ \\
\hline
\end{tabular}

\begin{tabular}{|c|c|c|}
\hline $\mathfrak{s}$ & $\boldsymbol{v}$ & $\mathfrak{s a}$ \\
\hline $\mathrm{S} 10_{\gamma \in[-1,0) \cup(0,1), \lambda \in \mathbb{R}}$ & $P$ & $\mathrm{~S} 40_{\lambda}$ \\
\hline $\mathrm{S} 10_{\gamma=0, \lambda \neq 0}$ & $P$ & S36 \\
\hline $\mathrm{S} 10_{\gamma=0, \lambda=0}$ & $P$ & S37 \\
\hline S16 & $B$ & S43 \\
\hline S19 & $B$ & S42 \\
\hline S20 & $B$ & S41 \\
\hline S23 & $B$ & S36 \\
\hline S24 & $B$ & S37 \\
\hline
\end{tabular}

\begin{tabular}{|l|l|l|}
\hline \multicolumn{1}{|c|}{$\mathfrak{s}$} & $\boldsymbol{v}$ & \multicolumn{1}{c|}{$\mathfrak{a}$} \\
\hline $\mathrm{S} 25$ & $\boldsymbol{B}$ & $\mathrm{S} 38$ \\
$\mathrm{~S} 25$ & $\boldsymbol{P}$ & $\mathrm{S} 38$ \\
$\mathrm{~S} 26$ & $\boldsymbol{B}$ & $\mathrm{S} 39$ \\
$\mathrm{~S} 26$ & $\boldsymbol{P}$ & $\mathrm{S} 38$ \\
S27 & $\boldsymbol{P}$ & $\mathrm{S} 41$ \\
S28 & $\boldsymbol{P}$ & $\mathrm{S} 42$ \\
S31 & $\boldsymbol{P}$ & $\mathrm{S} 38$ \\
S32 & $\boldsymbol{P}$ & $\mathrm{S}_{3}$ \\
S33 $_{\lambda \in \mathbb{R}}$ & $\boldsymbol{B}$ & S40 $_{\lambda}$ \\
\hline
\end{tabular}

Table 13. Correspondence between non-effective super Lie pairs and aristotelian superalgebras.

\subsection{Summary}

Table 14 lists the homogeneous superspaces we have classified in this paper. Each superspacetime is a superisation of an underlying spatially-isotropic, homogeneous (kinematical or aristotelian) spacetime, which we list in table 1, which is borrowed from [4] (see also [5]), to which we refer the reader for a detailed discussion of these spacetimes. Let us recall that table 1 is divided into five sections, corresponding to the different invariant structures which the homogeneous spacetimes admit, as recalled in the introduction. We have a similar division of table 14: with the superisations of spacetimes admitting a lorentzian, galilean, carrollian, aristotelian (with R-symmetries) and aristotelian (without R-symmetries) structures, respectively. All spacetimes admit superisations with the exception of the riemannian spaces, de Sitter spacetime $\left(\mathrm{dS}_{4}\right)$ and two of the carrollian spacetimes: carrollian de sitter (dSC) and the carrollian light-cone (LC).

\subsection{Low-rank invariants}

In this section, we exhibit the low-rank invariants of the homogeneous superspaces in table 14, all of which are reductive. Indeed, a homogeneous supermanifold with super Lie pair $(\mathfrak{s}, \mathfrak{h})$, where $\mathfrak{h} \subset \mathfrak{k}=\mathfrak{s}_{0}$, is reductive if and only if so is the underlying homogeneous manifold $(\mathfrak{k}, \mathfrak{h})$. This is because if $\mathfrak{k}=\mathfrak{h} \oplus \mathfrak{m}$ is a reductive split, then so is $\mathfrak{s}=\mathfrak{h} \oplus(\mathfrak{m} \oplus S)$, with $S=\mathfrak{s}_{\overline{1}}$ : the bracket $[\mathfrak{h}, \mathfrak{m}] \subset \mathfrak{m}$ because $(\mathfrak{k}, \mathfrak{h})$ is reductive and the bracket $[\mathfrak{h}, S] \subset S$ because $\mathfrak{h} \in \mathfrak{s}_{\overline{0}}$ and $S=\mathfrak{s}_{\overline{1}}$. In [4] it is shown that all the homogeneous spacetimes in table 1 are reductive with the exception of the carrollian light-cone LC, which in any case does not admit any (4|4)-dimensional superisation. Hence all the superspaces in table 14 are reductive.

Let $(\mathfrak{s}, \mathfrak{h})$ be the super Lie pair associated with one of the homogeneous superspaces in table 14 . We will write $\mathfrak{s}=\mathfrak{h} \oplus \mathfrak{m}$, where we have promoted $\mathfrak{m}$ to a vector superspace $\mathfrak{m}=\mathfrak{m}_{\overline{0}} \oplus \mathfrak{m}_{\overline{1}}$, with $\mathfrak{k}=\mathfrak{h} \oplus \mathfrak{m}_{\overline{0}}$ a reductive split and $\mathfrak{m}_{\overline{1}}=\mathfrak{s}_{\overline{1}}=S$.

Invariant tensors on the simply-connected superspace with super Lie pair $(\mathfrak{s}, \mathfrak{h})$ are in one-to-one correspondence with $\mathfrak{h}$-invariant tensors on $\mathfrak{m}$. Since $\mathfrak{h}$ contains the rotational subalgebra $\mathfrak{r} \cong \mathfrak{s o}(3), \mathfrak{h}$-invariant tensors are in particular also rotationally invariant. It is not difficult to write down the rotationally invariant tensors of low order. 


\begin{tabular}{|c|c|c|c|c|c|c|c|}
\hline SM\# & M & $\mathfrak{s}$ & $\mathfrak{k}($ or $\mathfrak{a})$ & th & $\mathbb{b}$ & $p$ & {$[\mathrm{Q}(s), \mathrm{Q}(s)]$} \\
\hline 1 & $M^{4}$ & S14 & K8 & & $\frac{1}{2} \mathbb{k}$ & & $|s|^{2} H-\mathrm{P}(s \mathbb{k} \bar{s})$ \\
\hline 2 & $\mathrm{AdS}_{4}$ & S15 & $\mathrm{K} 11$ & $\frac{1}{2} \oslash$ & $\frac{1}{2} \mathbb{k}$ & $\frac{1}{2} \curvearrowleft$ & $|s|^{2} H+\mathrm{J}(s \rrbracket \bar{s})+\mathrm{B}\left(s \bar{s}^{\circ}\right)-\mathrm{P}(s \llbracket \bar{s})$ \\
\hline 3 & G & S7 & $\mathrm{K} 2$ & $\mathbb{k}$ & & & $-\mathrm{P}(s \mathbb{k} \bar{s})$ \\
\hline 4 & G & S8 & $\mathrm{K} 2$ & & & & $-\mathrm{P}(s \mathbb{k} \bar{s})$ \\
\hline $5_{\lambda \in \mathbb{R}}$ & dSG & $\mathrm{S} 9_{-1, \lambda}$ & $\mathrm{K} 3-1$ & $\frac{1}{2}(1+\lambda \mathbb{k})$ & & & $-\frac{1}{2}(\mathrm{~B}(s \mathbb{k} \bar{s})-\mathrm{P}(s \mathbb{k} \bar{s}))$ \\
\hline $6_{\lambda \in \mathbb{R}}$ & dSG & $\mathrm{S} 10_{-1, \lambda}$ & $\mathrm{K} 3-1$ & $\frac{1}{2}(-1+\lambda \mathbb{k})$ & & & $-\frac{1}{2}(\mathrm{~B}(s \mathbb{k} \bar{s})+\mathrm{P}(s \mathbb{k} \bar{s}))$ \\
\hline $7_{\gamma \in(-1,1), \lambda \in \mathbb{R}}$ & $\mathrm{dSG}_{\gamma}$ & $\mathrm{S} 9_{\gamma, \lambda}$ & $\mathrm{K} 3 \gamma$ & $\frac{1}{2}(1+\lambda \mathbb{k})$ & & & $\frac{1}{1-\gamma}(\gamma \mathrm{B}(s \mathbb{k} \bar{s})+\mathrm{P}(s \mathbb{k} \bar{s}))$ \\
\hline $8_{\gamma \in(-1,1), \lambda \in \mathbb{R}}$ & $\mathrm{dSG}_{\gamma}$ & $\mathrm{S} 10_{\gamma, \lambda}$ & $\mathrm{K} 3 \gamma$ & $\frac{1}{2}(\gamma+\lambda \mathbb{k})$ & & & $\frac{1}{\gamma-1}(\mathrm{~B}(s \llbracket \bar{s})+\mathrm{P}(s \llbracket \bar{s}))$ \\
\hline $9_{\lambda \in \mathbb{R}}$ & $\mathrm{dSG}_{\gamma=1}$ & $\mathrm{~S} 12_{\lambda}$ & $\mathrm{K}_{3}$ & $\frac{1}{2}(1+\lambda \mathbb{k})$ & & & $\mathrm{B}(s \mathbb{k} \bar{s})+\mathrm{P}(s \mathbb{k} \bar{s})$ \\
\hline 10 & AdSG & $\mathrm{S} 11_{0}$ & $\mathrm{~K} 4_{0}$ & $\frac{1}{2} §$ & & & $-\mathrm{B}(s \circ \bar{s})+\mathrm{P}(s \mathbb{k} \bar{s})$ \\
\hline $11_{\chi>0}$ & $\mathrm{AdSG}_{\chi}$ & $\mathrm{S} 11_{\chi}$ & $\mathrm{K} 4 \chi$ & $\frac{1}{2}(\chi+§)$ & & & $\mathrm{B}(s \mathbb{k}(\chi+\jmath) \bar{s})+\mathrm{P}(s \mathbb{k} \bar{s})$ \\
\hline 12 & C & $\mathrm{S} 13$ & K6 & & & & $|s|^{2} H$ \\
\hline 13 & AdSC & S14 & $\mathrm{K} 8$ & & & $\frac{1}{2} \AA$ & $|s|^{2} H+\mathrm{B}(s \mathrm{\circ} \bar{s})$ \\
\hline 14 & $\mathbb{R} \times H^{3}$ & S17 & $\mathrm{K} 12$ & & $\frac{1}{2}$ & & $|s|^{2} H$ \\
\hline 15 & $\mathbb{R} \times H^{3}$ & S18 & $\mathrm{K} 12$ & $\frac{1}{2} k$ & $\frac{1}{2}$ & & $|s|^{2} H-\mathrm{B}(s \mathbb{k} \bar{s})$ \\
\hline 16 & $\mathbb{R} \times S^{3}$ & S21 & $\mathrm{K} 13$ & & $\frac{1}{2}$ & & $|s|^{2} H$ \\
\hline 17 & $\mathbb{R} \times S^{3}$ & S22 & $\mathrm{K} 13$ & $\frac{1}{2} \mathbb{k}$ & $\frac{1}{2}$ & & $|s|^{2} H-\mathrm{B}(s \mathbb{k} \bar{s})$ \\
\hline 18 & S & S27 & K14 & & $\frac{1}{2}$ & & $|s|^{2} H$ \\
\hline 19 & $\mathrm{~S}$ & $\mathrm{~S} 28$ & K14 & $\frac{1}{2} \mathbb{k}$ & $\frac{1}{2}$ & & $|s|^{2} H-\mathrm{B}(s \llbracket \bar{s})$ \\
\hline 20 & S & S36 & $\mathrm{A} 1$ & k & - & & $-\mathrm{P}(s \mathbb{k} \bar{s})$ \\
\hline 21 & S & S37 & $\mathrm{A} 1$ & & - & & $-\mathrm{P}(s \llbracket \bar{s})$ \\
\hline 22 & S & S38 & $\mathrm{A} 1$ & & - & & $|s|^{2} H$ \\
\hline 23 & S & S39 & $\mathrm{A} 1$ & & - & & $|s|^{2} H-\mathrm{P}(s \mathbb{k} \bar{s})$ \\
\hline $24_{\lambda \in \mathbb{R}}$ & TS & $S 40_{\lambda}$ & $\mathrm{A} 2$ & $\frac{1}{2}(1+\lambda \mathbb{k})$ & - & & $-\mathrm{P}(s \mathbb{k} \bar{s})$ \\
\hline 25 & $\mathbb{R} \times S^{3}$ & S41 & $\mathrm{A} 3_{+}$ & & - & $\frac{1}{2}$ & $|s|^{2} H$ \\
\hline 26 & $\mathbb{R} \times S^{3}$ & S42 & $\mathrm{A} 3_{+}$ & $\mathbb{k}$ & - & $\frac{1}{2}$ & $|s|^{2} H-\mathrm{J}(s \mathbb{k} \bar{s})-\mathrm{P}(s \mathbb{k} \bar{s})$ \\
\hline 27 & $\mathbb{R} \times H^{3}$ & S43 & $\mathrm{A} 3_{-}$ & & - & $\frac{1}{2} \AA$ & $\mathrm{J}\left(s \rho_{\bar{s}} \bar{s}\right)-\mathrm{P}(s \mathbb{k} \bar{s})$ \\
\hline
\end{tabular}

Table 14. Simply-connected spatially-isotropic homogeneous superspaces.

The first column is our identifier for the superspace, whereas the second column is the underlying homogeneous spacetime it superises. The next two columns are the isomorphism classes of kinematical Lie superalgebra and kinematical Lie algebra, respectively. The next columns specify the brackets of $\mathfrak{s}$ not of the form $[\boldsymbol{J},-]$ in a basis where $\mathfrak{h}$ is spanned by $\boldsymbol{J}$ and $\boldsymbol{B}$. As explained in section 2.2, supercharges $\mathrm{Q}(s)$ are parametrised by $s \in \mathbb{H}$, whereas $\mathrm{J}(\omega), \mathrm{B}(\beta)$ and $\mathrm{P}(\pi)$ are parametrised by $\omega, \beta, \pi \in \operatorname{Im} \nVdash$. The brackets are given by $[H, \mathrm{Q}(s)]=\mathrm{Q}(s \mathfrak{h}),[\mathrm{B}(\beta), \mathrm{Q}(s)]=\mathrm{Q}(\beta s \mathfrak{b})$ and $[\mathrm{P}(\pi), \mathrm{Q}(s)]=\mathrm{Q}(\pi s \mathbb{p})$, for some $\mathbb{h}, \mathbb{b}, \mathbb{p} \in \mathbb{H}$. The table is divided into five sections from top to bottom: lorentzian, galilean, carrollian, aristotelian with R-symmetries and aristotelian.

As an $\mathfrak{r}$-module, $\mathfrak{m}=\mathbb{R} \oplus V \oplus S$, where $\mathbb{R}$ is the trivial one-dimensional representation, $V$ is the vector three-dimensional representation and $S$ is the spinor four-dimensional representation. Under the isomorphism $\mathfrak{r}=\mathfrak{s p}(1)=\operatorname{Im} \mathbb{H}, \mathfrak{m}=\mathbb{R} \oplus \operatorname{Im} H \oplus \mathbb{H}$, where the 
integrated action of a unit-norm quaternion $u \in \operatorname{Sp}(1)$ on $(h, p, s) \in \mathfrak{m}$ is given by

$$
u \cdot(h, p, s)=(h, u p \bar{u}, u s) .
$$

Let $H, P_{i}, Q_{a}$ denote a basis for $\mathfrak{m}$, where $P_{i}$ and $Q_{a}$ have been defined in equation (2.4). We let $\eta, \pi^{i}, \theta^{a}$ denote the canonically dual basis for $\mathfrak{m}^{*}$. There is a rotationally invariant line in $\mathfrak{m}$ : namely, the span of $H$, which lives in $\mathfrak{m}_{\overline{0}}$. Dually, there is a rotationally invariant line in $\mathfrak{m}^{*}$, which is the span of $\eta$. These are all the rotationally invariant tensors of rank 1.

Let us now consider rank 2. As a representation of $\operatorname{Sp}(1), \mathfrak{m} \otimes \mathfrak{m}$ has the following invariants. First of all, we have $H^{2}$, which is the only invariant featuring $H$. Another invariant is $P^{2}:=\sum_{i} P_{i} \otimes P_{i}$, which corresponds to the $\mathrm{Sp}(1)$-invariant inner product $\langle-,-\rangle: \operatorname{Im} \mathbb{H} \times \operatorname{Im} \mathbb{H} \rightarrow \mathbb{R}$ given by $\langle\alpha, \beta\rangle=\operatorname{Re}(\alpha \bar{\beta})=-\operatorname{Re}(\alpha \beta)$. If $q \in \mathbb{H}$ is any quaternion, the real bilinear form

$$
\omega_{q}: \mathbb{H} \rightarrow \mathbb{R} \quad \text { defined by } \quad \omega_{q}\left(s_{1}, s_{2}\right)=\operatorname{Re}\left(s_{1} q \bar{s}_{2}\right)
$$

is $\mathrm{Sp}(1)$-invariant: symmetric if $q$ is real and symplectic if $q$ is imaginary (and nonzero). This gives rise to four $\operatorname{Sp}(1)$-invariants quadratic in $Q: \sum_{a} Q_{a} \otimes Q_{a}$ and the triplet $\sum_{a, b} I_{a b} Q_{a} \otimes Q_{b}, \sum_{a, b} J_{a b} Q_{a} \otimes Q_{b}$ and $\sum_{a, b} K_{a b} Q_{a} \otimes Q_{b}$, where $I, J, K$ are the matrices representing right-multiplication by the quaternions $\stackrel{\AA}{0}, \mathfrak{j}$, $\mathbb{k}$; that is,

$$
\mathrm{Q}\left(s^{\circ}\right)=\sum_{a, b=1}^{4} Q_{a} I_{a b} s_{b}, \quad \mathrm{Q}(s \AA)=\sum_{a, b=1}^{4} Q_{a} J_{a b} s_{b} \quad \text { and } \quad \mathrm{Q}(s \mathbb{k})=\sum_{a, b=1}^{4} Q_{a} K_{a b} s_{b} .
$$

Similarly there are several rotational invariants in $\mathfrak{m}^{*} \otimes \mathfrak{m}^{*}: \eta^{2}$ and, in addition, the symmetric tensors $\pi^{2}$ and $\theta^{2}$, and the triplet of symplectic forms $\omega_{I}, \omega_{J}$ and $\omega_{K}$, defined as follows:

$$
\begin{aligned}
\pi^{2}\left(\mathrm{P}\left(\alpha^{\prime}\right), \mathrm{P}(\alpha)\right) & =\operatorname{Re}\left(\alpha^{\prime} \bar{\alpha}\right)=-\operatorname{Re}\left(\alpha^{\prime} \alpha\right) \\
\theta^{2}\left(\mathrm{Q}\left(s^{\prime}\right), \mathrm{Q}(s)\right) & =\operatorname{Re}\left(s^{\prime} \bar{s}\right) \\
\omega_{I}\left(\mathrm{Q}\left(s^{\prime}\right), \mathrm{Q}(s)\right) & =\operatorname{Re}\left(s^{\prime} \circ \bar{s}\right) \\
\omega_{J}\left(\mathrm{Q}\left(s^{\prime}\right), \mathrm{Q}(s)\right) & =\operatorname{Re}\left(s^{\prime} \oslash \bar{s}\right) \\
\omega_{K}\left(\mathrm{Q}\left(s^{\prime}\right), \mathrm{Q}(s)\right) & =\operatorname{Re}\left(s^{\prime} \llbracket \bar{k}\right) .
\end{aligned}
$$

To investigate the invariant tensors on $(\mathfrak{s}, \mathfrak{h})$ we need to investigate the action of $\boldsymbol{B}$ on the tensors. For the classical invariants (i.e., those not involving $Q_{a}$ or $\theta^{a}$ ), we may consult [4]: the lorentzian metric (and the corresponding cometric) are invariant for the lorentzian spacetimes, the clock one-form and spatial cometric for the galilean spacetimes, the carrollian vector and the spatial metric for the carrollian spacetimes. The generators $\boldsymbol{B}$ act trivially on aristotelian spacetimes, so the rotationally invariant tensors are the invariant tensors. For the invariants involving $Q_{a}$ or $\theta^{a}$, we need to examine how $\boldsymbol{B}$ acts on $S$.

As can be gleaned from table $14, \boldsymbol{B}$ acts trivially on $\boldsymbol{Q}$ in most cases. The exceptions are Minkowski and AdS superspaces and the aristotelian superspaces where $\boldsymbol{B}$ acts via 
R-symmetries. Hence in all other superspaces, the four rotational invariants in $\mathfrak{m}_{\overline{1}} \otimes \mathfrak{m}_{\overline{1}}$ defined above and $\theta^{2}, \omega_{I}, \omega_{J}$ and $\omega_{K}$ in $\mathfrak{m}_{\overline{1}}^{*} \otimes \mathfrak{m}_{\overline{1}}^{*}$ are $\mathfrak{h}$-invariant. This situation continues to hold for the aristotelian superspaces with R-symmetry, namely SM14-SM19. Indeed, one can show that all the rotational invariants which are quadratic in $\boldsymbol{Q}$ or in the $\theta^{a}$ are also R-symmetry invariant. Indeed, the R-symmetry generator $B_{i}$ acts on $\mathfrak{m}_{\overline{1}}$ in the same way as the infinitesimal rotation generator $J_{i}$.

Hence it is only for Minkowski and AdS superspaces that the $\mathfrak{h}$-invariants do not agree with the $\mathfrak{r}$-invariants. For both of these superspaces, $\mathfrak{h} \cong \mathfrak{s o}(3,1)$, acting in the same way on the spinors:

$$
[\mathrm{B}(\beta), \mathrm{Q}(s)]=\mathrm{Q}\left(\frac{1}{2} \beta s \mathbb{k}\right)
$$

It is a simple calculation to see that the following are $\mathfrak{h}$-invariant: $\sum_{a, b} I_{a b} Q_{a} \otimes Q_{b}$, $\sum_{a, b} J_{a b} Q_{a} \otimes Q_{b}, \omega_{I}$ and $\omega_{J}$.

Since $\mathfrak{h}$ is isomorphic to the Lorentz subalgebra, we recover the well-known fact that there are two independent Lorentz-invariant symplectic structures on the Majorana spinors. This does not contradict the fact that the Majorana spinor representation $S$ of $\mathfrak{s o}(3,1)$ is irreducible as a real representation, since its complexification (the Dirac spinor representation) decomposes as a direct sum of the two Weyl spinor representations, each one having a Lorentz-invariant symplectic structure.

\section{Limits between superspaces}

In this section, we exhibit some limits between the superspaces in table 14 and interpret them in terms of contractions of the underlying Lie superalgebras.

As we will show, a limit between two superspaces induces a limit of the underlying homogeneous spacetimes. These were determined in [4]. Our discussion will closely follow that in ([4], section 5). There contractions of a Lie algebra $\mathfrak{g}=(V, \phi)$, where $V$ is a finite-dimensional real vector space and $\phi: \wedge^{2} V \rightarrow V$ is a linear map satisfying the Jacobi identity, were defined as limits of curves in the space of Lie brackets. If $g:(0,1] \rightarrow \operatorname{GL}(V)$, mapping $t \mapsto g_{t}$, is a continuous curve with $g_{1}=\mathbb{1}_{V}$, we can define a curve of isomorphic Lie algebras $\left(V, \phi_{t}\right)$, where

$$
\phi_{t}(X, Y):=\left(g_{t}^{-1} \cdot \phi\right)(X, Y)=g_{t}^{-1}\left(\phi\left(g_{t} X, g_{t} Y\right)\right) \text {. }
$$

If the limit $\phi_{0}=\lim _{t \rightarrow 0} \phi$ exists, it defines a Lie algebra $\mathfrak{g}_{0}=\left(V, \phi_{0}\right)$ which is then a contraction of $\mathfrak{g}=\left(V, \phi_{1}\right)$.

In the current case, we will contract Lie superalgebras $\mathfrak{s}=(V, \phi)$, where $V$ is now a real finite-dimensional super vector space and $\phi: \wedge^{2} V \rightarrow V$ is a linear map, where $\wedge^{2}$ is defined in the super sense, satisfying the super-Jacobi identity. We will define contractions of $\mathfrak{s}$ in a completely analogous manner. 


\subsection{Contractions of the AdS superalgebra}

We begin with the superalgebra for the AdS superspace SM2, whose generators $\boldsymbol{J}, \boldsymbol{B}, \boldsymbol{P}$, $H$ and $\boldsymbol{Q}$ satisfy the following brackets (in shorthand notation):

$$
\begin{array}{llll}
{[\boldsymbol{J}, \boldsymbol{J}]} & =\boldsymbol{J} & {[H, \boldsymbol{B}]=-\boldsymbol{P}} & \\
{[\boldsymbol{J}, \boldsymbol{B}]=\boldsymbol{B}} & {[H, \boldsymbol{P}]=\boldsymbol{B}} & & {[\boldsymbol{B}, \boldsymbol{Q}]=\boldsymbol{Q}} \\
{[\boldsymbol{J}, \boldsymbol{P}]=\boldsymbol{P}} & {[\boldsymbol{B}, \boldsymbol{P}]=H} & {[\boldsymbol{P}, \boldsymbol{Q}]=\boldsymbol{Q}} \\
{[\boldsymbol{J}, \boldsymbol{Q}]=\boldsymbol{Q}} & {[\boldsymbol{B}, \boldsymbol{B}]=-\boldsymbol{J}} & {[\boldsymbol{Q}, \boldsymbol{Q}]=H+\boldsymbol{J}+\boldsymbol{B}-\boldsymbol{P} .}
\end{array}
$$

Consider the following three-parameter family of linear transformations $g_{\kappa, c, \tau}$ defined by

$$
g_{\kappa, c, \tau} \cdot \boldsymbol{J}=\boldsymbol{J}, \quad g_{\kappa, c, \tau} \cdot \boldsymbol{B}=\frac{\tau}{c} \boldsymbol{B}, \quad g_{\kappa, c, \tau} \cdot \boldsymbol{P}=\frac{\kappa}{c} \boldsymbol{P}, \quad g_{\kappa, c, \tau} \cdot H=\tau \kappa H, \quad g_{\kappa, c, \tau} \cdot \boldsymbol{Q}=\frac{\kappa \tau}{c} \boldsymbol{Q} .
$$

The action on the even generators is as in ([4], section 5) and the action on $\boldsymbol{Q}$ is chosen to ensure that the bracket $[\boldsymbol{Q}, \boldsymbol{Q}]$ has well-defined limits as $\kappa \rightarrow 0, c \rightarrow \infty$ or $\tau \rightarrow 0$.

The brackets involving $\boldsymbol{J}$ remain unchanged for the above transformations and the remaining brackets become

$$
\begin{aligned}
& {[H, \boldsymbol{B}]=-\tau^{2} \boldsymbol{P} \quad[\boldsymbol{B}, \boldsymbol{B}]=-\frac{\tau^{2}}{c^{2}} \boldsymbol{J} \quad[\boldsymbol{B}, \boldsymbol{Q}]=\frac{\tau}{c} \boldsymbol{Q}} \\
& {[H, \boldsymbol{P}]=\kappa^{2} \boldsymbol{B} \quad[\boldsymbol{P}, \boldsymbol{P}]=-\frac{\kappa^{2}}{c^{2}} \boldsymbol{J} \quad[\boldsymbol{P}, \boldsymbol{Q}]=\frac{\kappa}{c} \boldsymbol{Q}} \\
& {[\boldsymbol{B}, \boldsymbol{P}]=\frac{1}{c^{2}} H \quad[H, \boldsymbol{Q}]=\kappa \tau \boldsymbol{Q} \quad[\boldsymbol{Q}, \boldsymbol{Q}]=\frac{1}{c} H+\frac{\kappa \tau}{c} \boldsymbol{J}+\kappa \boldsymbol{B}-\tau \boldsymbol{P} .}
\end{aligned}
$$

We now want to take the limits $\kappa \rightarrow 0, c \rightarrow \infty$, and $\tau \rightarrow 0$ in turn, corresponding to the flat, non-relativistic, and ultra-relativistic limits, respectively. Notice that the limits of the brackets between the even generators will produce the same Lie algebra contractions as in [4]. Thus we cannot have a limit from one superspace to another unless there exists a limit between their underlying homogeneous spacetimes.

Taking the flat limit $\kappa \rightarrow 0$, we are left with

$$
[H, \boldsymbol{B}]=-\tau^{2} \boldsymbol{P}, \quad[\boldsymbol{B}, \boldsymbol{P}]=\frac{1}{c^{2}} H, \quad[\boldsymbol{B}, \boldsymbol{B}]=-\frac{\tau^{2}}{c^{2}} \boldsymbol{J}, \quad[\boldsymbol{B}, \boldsymbol{Q}]=\frac{\tau}{c} \boldsymbol{Q} \text { and }[\boldsymbol{Q}, \boldsymbol{Q}]=\frac{1}{c} H-\tau \boldsymbol{P} .
$$

For $\frac{\tau}{c} \neq 0$, this is the Poincaré superalgebra (S14). Thus, we obtain the limit SM2 $\rightarrow$ SM1. Subsequently taking the non-relativistic limit $c \rightarrow \infty$, the brackets reduce to

$$
[H, \boldsymbol{B}]=-\tau^{2} \boldsymbol{P} \quad \text { and } \quad[\boldsymbol{Q}, \boldsymbol{Q}]=-\tau \boldsymbol{P} .
$$

For $\tau \neq 0$, this shows us that we have the limit SM1 $\rightarrow$ SM4.

Alternatively, we could have taken the ultra-relativistic limit $\tau \rightarrow 0$, which, for $c \neq 0$, gives us the Carroll superalgebra (S13):

$$
[\boldsymbol{B}, \boldsymbol{P}]=\frac{1}{c^{2}} H \quad \text { and } \quad[\boldsymbol{Q}, \boldsymbol{Q}]=\frac{1}{c} H .
$$

Thus, we have SM1 $\rightarrow$ SM12. 
Returning to the AdS superalgebra (S15) and taking the non-relativistic limit $c \rightarrow \infty$, we find

$$
[H, \boldsymbol{B}]=-\tau^{2} \boldsymbol{P}, \quad[H, \boldsymbol{P}]=\kappa^{2} \boldsymbol{B}, \quad[H, \boldsymbol{Q}]=\kappa \tau \boldsymbol{Q} \quad \text { and } \quad[\boldsymbol{Q}, \boldsymbol{Q}]=\kappa \boldsymbol{B}-\tau \boldsymbol{P} .
$$

For $\tau \kappa \neq 0$, this is $\mathrm{S}_{1} 1_{0}$ (under a suitable basis change). Therefore, we have SM2 $\rightarrow \mathrm{SM} 10$. Because these limits commute, we may now take the flat limit to arrive at SM4.

Finally, we may take the ultra-relativistic limit of AdS (S15). This limit leaves the brackets

$$
[H, \boldsymbol{P}]=\kappa^{2} \boldsymbol{B}, \quad[\boldsymbol{B}, \boldsymbol{P}]=\frac{1}{c^{2}} H, \quad[\boldsymbol{P}, \boldsymbol{P}]=-\frac{\kappa^{2}}{c^{2}} \boldsymbol{J}, \quad[\boldsymbol{P}, \boldsymbol{Q}]=\frac{\kappa}{c} \boldsymbol{Q} \text { and }[\boldsymbol{Q}, \boldsymbol{Q}]=\frac{1}{c} H+\kappa \boldsymbol{B},
$$

for $\frac{\kappa}{c} \neq 0$. Thus, we arrive at SM13. Subsequently taking the flat limit, we find SM12, as expected.

We can also take limits from the superspaces discussed above to non-effective super Lie pairs, which will have associated aristotelian superspaces. Since all of the above superspaces have either SM4 or SM12 as a limit, we will only show the limits to aristotelian superspaces coming form these two cases. Beginning with SM4, we can use the transformation

$$
g_{t} \cdot \boldsymbol{B}=t \boldsymbol{B}, \quad g_{t} \cdot H=H, \quad g_{t} \cdot \boldsymbol{P}=\boldsymbol{P} \quad \text { and } \quad g_{t} \cdot \boldsymbol{Q}=\boldsymbol{Q}
$$

and the limit $t \rightarrow 0$ to obtain SM21. Using the same transformation and limit, we can also start with SM12 and find SM22.

\subsection{Remaining galilean superspaces}

We have shown that we obtain the other lorentzian and two carrollian superspaces as limits of the AdS superspace SM2: namely, Minkowski (SM1), Carroll (SM12) and carrollian anti de Sitter (SM13) superspaces. In addition, we also obtain two superisations of galilean spacetimes: a superisation SM4 of the flat galilean spacetime and the superisation SM10 of galilean anti de Sitter spacetime. But what about the superisations of other galilean spacetimes?

\subsubsection{Flat galilean superspaces}

From SM2 we obtained the galilean superspace SM4. There is a second superisation SM3 of the flat galilean homogeneous spacetime, from which we can also reach SM4. Indeed, using the transformations

$$
g_{t} \cdot \boldsymbol{B}=t \boldsymbol{B}, \quad g_{t} \cdot H=t H, \quad g_{t} \cdot \boldsymbol{P}=t \boldsymbol{P} \quad \text { and } \quad g_{t} \cdot \boldsymbol{Q}=\sqrt{t} \boldsymbol{Q},
$$

on the Lie superalgebra for SM3, and taking the limit $t \rightarrow 0$, we find the Lie superalgebra for SM4. Thus, we have SM3 $\rightarrow$ SM4.

Beginning with SM3, we may also consider the transformation

$$
g_{t} \cdot \boldsymbol{B}=t \boldsymbol{B}, \quad g_{t} \cdot H=H, \quad g_{t} \cdot \boldsymbol{P}=t \boldsymbol{P} \quad \text { and } \quad g_{t} \cdot \boldsymbol{Q}=\sqrt{t} \boldsymbol{Q},
$$

and the limit $t \rightarrow 0$. This procedure will give us a non-effective super Lie pair corresponding to SM20. 


\subsubsection{Galilean de Sitter superspaces}

The superspaces SM5 ${ }_{\lambda}$ and $\mathrm{SM} 6_{\lambda}$ arise as the $\gamma \rightarrow-1$ limit of $\mathrm{SM} 7_{\gamma, \lambda}$ and $\mathrm{SM} 8_{\gamma, \lambda}$, respectively. This fact has already been noted in section 4.3.2. Section 4.3.3 demonstrated that $\mathrm{SM} 9_{\lambda}$ is the $\gamma \rightarrow 1$ limit of $\mathrm{SM} 7_{\gamma, \lambda}$ and $\mathrm{SM} 8_{\gamma, \lambda}$.

The superalgebras associated with these five superspaces take the general form

$$
\begin{aligned}
{[H, \mathrm{~B}(\beta)] } & =-\mathrm{P}(\beta) & {[H, \mathrm{Q}(s)] } & =\frac{1}{2} \mathrm{Q}(s(\eta+\lambda \mathbb{k})) \\
{[H, \mathrm{P}(\pi)] } & =\gamma \mathrm{B}(\pi)+(1+\gamma) \mathrm{P}(\pi) & {[\mathrm{Q}(s), \mathrm{Q}(s)] } & =\rho \mathrm{B}(s \llbracket \bar{s})+\sigma \mathrm{P}(s k \bar{s})
\end{aligned}
$$

for some $\eta, \rho, \sigma \in \mathbb{R}$, where $\gamma \in[-1,1]$ and $\lambda \in \mathbb{R}$ are the parameters of the Lie superalgebras. Using the transformations

$$
g_{t} \cdot \boldsymbol{B}=\boldsymbol{B}, \quad g_{t} \cdot H=t H, \quad g_{t} \cdot \boldsymbol{P}=t \boldsymbol{P} \quad \text { and } \quad g_{t} \cdot \boldsymbol{Q}=\sqrt{\omega t} \boldsymbol{Q},
$$

where $\omega \in \mathbb{R}$, and taking the limit $t \rightarrow 0$, the above brackets become

$$
[H, \mathrm{~B}(\beta)]=-\mathrm{P}(\beta) \quad \text { and } \quad[\mathrm{Q}(s), \mathrm{Q}(s)]=\omega \sigma \mathrm{P}(s \llbracket \bar{s}) .
$$

Therefore, by choosing $\omega=-\sigma^{-1}$, we can always recover SM4.

There is a second superisation of the flat galilean homogeneous spacetime, namely SM3. There does not seem to be any Lie-superalgebra contraction that gives SM3, but as we will see below, there are non-contracting limits (involving taking $\lambda \rightarrow \pm \infty$ ) which take the superspaces $\mathrm{SM} 5_{\lambda}, \mathrm{SM} 6_{\lambda}, \mathrm{SM} 7_{\gamma, \lambda}, \mathrm{SM} 8_{\gamma, \lambda}$ and $\mathrm{SM} 9_{\lambda}$ to $\mathrm{SM} 3$.

\subsubsection{Galilean anti de Sitter superspaces}

The superspace SM10 is, by definition, the $\chi \rightarrow 0$ limit of SM11 $1_{\chi}$. These algebras take the form

$$
\begin{aligned}
{[H, \mathrm{~B}(\beta)] } & =-\mathrm{P}(\beta) & {[H, \mathrm{Q}(s)] } & =\frac{1}{2} \mathrm{Q}(s(\chi+\jmath)) \\
{[H, \mathrm{P}(\pi)] } & =\left(1+\chi^{2}\right) \mathrm{B}(\pi)+\chi \mathrm{P}(\pi) & {[\mathrm{Q}(s), \mathrm{Q}(s)] } & =-\mathrm{B}(s \mathrm{~s} \bar{s})-\mathrm{P}(s \llbracket \bar{s}),
\end{aligned}
$$

where $\chi \geq 0$ is the parameter of the Lie superalgebra. Using the same transformations as in the galilean de Sitter case, but with $\omega=1$, we find

$$
[H, \mathrm{~B}(\beta)]=\mathrm{P}(\beta) \quad \text { and } \quad[\mathrm{Q}(s), \mathrm{Q}(s)]=-\mathrm{P}(s \mathbb{k} \bar{s}) .
$$

Thus, we find SM4 as a limit of both SM10 and SM11 .

We cannot obtain SM3 as a limit of these superspaces as SM3 has collinear $\mathbb{h}$ and $\mathbb{C}_{3}$, whereas $\mathrm{SM} 10$ and $\mathrm{SM} 11_{\chi}$ have orthogonal th and $\mathbb{C}_{3}$.

\subsubsection{Non-contracting limits}

In [4] it was shown that $\lim _{\chi \rightarrow \infty} \mathrm{AdSG}_{\chi}=\mathrm{dSG}_{1}$, but this limit is not induced by a Lie algebra contraction since the Lie algebras are non-isomorphic for different values of $\chi$. Does this limit extend to the superspaces?

Beginning with $\mathrm{SM}_{1} 1_{\chi}$, change basis such that

$$
H^{\prime}=\chi^{-1} H, \quad \boldsymbol{B}^{\prime}=\boldsymbol{B}, \quad \boldsymbol{P}^{\prime}=\chi^{-1} \boldsymbol{P} \quad \text { and } \quad \boldsymbol{Q}^{\prime}=\chi^{-1 / 2} \boldsymbol{Q},
$$


under which the brackets become

$$
\begin{aligned}
{\left[H^{\prime}, \mathrm{B}^{\prime}(\beta)\right] } & =-\mathrm{P}^{\prime}(\beta) & {\left[H^{\prime}, \mathrm{Q}^{\prime}(s)\right] } & =\frac{1}{2 \chi} \mathrm{Q}^{\prime}(s(\chi+\AA)) \\
{\left[H^{\prime}, \mathrm{P}^{\prime}(\pi)\right] } & =2 \mathrm{P}^{\prime}(\pi)+\left(1+\chi^{-2}\right) \mathrm{B}^{\prime}(\pi) & {\left[\mathrm{Q}^{\prime}(s), \mathrm{Q}^{\prime}(s)\right] } & =-\chi^{-1} \mathrm{~B}^{\prime}(s i \bar{s})+\mathrm{B}^{\prime}(s \llbracket \bar{s})+\mathrm{P}(s \llbracket \bar{s}) .
\end{aligned}
$$

Taking the limit $\chi \rightarrow \infty$, we find

$$
\begin{aligned}
{\left[H^{\prime}, \mathrm{B}^{\prime}(\beta)\right] } & =-\mathrm{P}^{\prime}(\beta) & {\left[H^{\prime}, \mathrm{Q}^{\prime}(s)\right] } & =\frac{1}{2} \mathrm{Q}^{\prime}(s) \\
{\left[H^{\prime}, \mathrm{P}^{\prime}(\pi)\right] } & =2 \mathrm{P}^{\prime}(\pi)+\mathrm{B}^{\prime}(\pi) & {\left[\mathrm{Q}^{\prime}(s), \mathrm{Q}^{\prime}(s)\right] } & =-\mathrm{B}^{\prime}(s \mathbb{k} \bar{s})+\mathrm{P}(s \mathbb{k} \bar{s}) .
\end{aligned}
$$

This Lie superalgebra is precisely that for $\mathrm{SM}_{0}$. Thus, we inherit this limit from the underlying homogeneous spacetimes.

The superspaces $\mathrm{SM}_{\lambda}, \mathrm{SM}_{\lambda}, \mathrm{SM} 7_{\gamma, \lambda}, \mathrm{SM} 8_{\gamma, \lambda}$ and $\mathrm{SM} 9_{\lambda}$ all have an additional parameter $\lambda$ and we can ask what happens if we take the limit $\lambda \rightarrow \pm \infty$ in these cases. This is again a non-contracting limit, since the Lie superalgebras with different values of $\lambda \in \mathbb{R}$ are not isomorphic.

Using the general form of the brackets stated in (5.13) above, consider a change of basis

$$
\boldsymbol{B}^{\prime}=\boldsymbol{B}, \quad H^{\prime}=2 \lambda^{-1} H, \quad \boldsymbol{P}^{\prime}=2 \lambda^{-1} \boldsymbol{P} \quad \text { and } \quad \boldsymbol{Q}^{\prime}=\lambda^{-\frac{1}{2}} \boldsymbol{Q} .
$$

In our new basis, the brackets become

$$
\begin{aligned}
{\left[H^{\prime}, \mathrm{B}^{\prime}(\beta)\right] } & =-\mathrm{P}^{\prime}(\beta) & {\left[H^{\prime}, \mathrm{Q}^{\prime}(s)\right] } & =\mathrm{Q}^{\prime}\left(s\left(\lambda^{-1} \eta+\mathbb{k}\right)\right) \\
{\left[H^{\prime}, \mathrm{P}^{\prime}(\pi)\right] } & =4 \lambda^{-2} \gamma \mathrm{B}^{\prime}(\pi)+2 \lambda^{-1}(1+\gamma) \mathrm{P}^{\prime}(\pi) & {\left[\mathrm{Q}^{\prime}(s), \mathrm{Q}^{\prime}(s)\right] } & =\lambda^{-1} \rho \mathrm{B}^{\prime}(s k \bar{s})+\frac{\sigma}{2} \mathrm{P}^{\prime}(s \mathbb{k} \bar{s}) .
\end{aligned}
$$

Taking either $\lambda \rightarrow \infty$ or $\lambda \rightarrow-\infty$, we find

$$
\left[H^{\prime}, \mathrm{B}^{\prime}(\beta)\right]=-\mathrm{P}^{\prime}(\beta), \quad\left[H^{\prime}, \mathrm{Q}^{\prime}(s)\right]=\mathrm{Q}^{\prime}(s \mathbb{k}), \quad\left[\mathrm{Q}^{\prime}(s), \mathrm{Q}^{\prime}(s)\right]=\frac{\sigma}{2} \mathrm{P}^{\prime}(s \mathbb{k} \bar{s}) .
$$

Rescaling both $\boldsymbol{B}^{\prime}$ and $\boldsymbol{P}^{\prime}$ by $\frac{\sigma}{2}$, we recover the Lie superalgebra for SM3.

Figure 1 below illustrates the different superspaces and the limits between them. The families $\mathrm{SM} 5_{\lambda}, \mathrm{SM} 6_{\lambda}, \mathrm{SM} 7_{\gamma, \lambda}, \mathrm{SM} 8_{\gamma, \lambda}$ and $\mathrm{SM} 9_{\lambda}$ fit together into a two-dimensional space which also includes SM3 as their common limits $\lambda \rightarrow \pm \infty$ and which can be described as follows. If we fix $\lambda \in \mathbb{R}$, then

$$
\lim _{\gamma \rightarrow 1} \mathrm{SM} 7_{\gamma, \lambda}=\mathrm{SM} 9_{\lambda} \quad \text { whereas } \quad \lim _{\gamma \rightarrow-1} \mathrm{SM} 7_{\gamma, \lambda}=\mathrm{SM} 5_{\lambda} .
$$

Similarly, again fixing $\lambda \in \mathbb{R}$, we have

$$
\lim _{\gamma \rightarrow 1} \mathrm{SM}_{\gamma, \lambda}=\mathrm{SM} 9_{\lambda} \quad \text { whereas } \quad \lim _{\gamma \rightarrow-1} \mathrm{SM} 8_{\gamma, \lambda}=\mathrm{SM} 6_{\lambda} .
$$

This gives rise to the following two-dimensional parameter spaces:

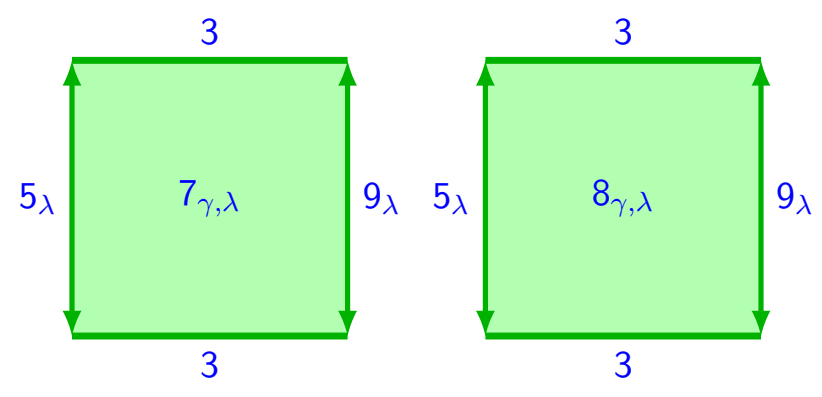


We then flip the square on the right horizontally and glue the two squares along their common $9_{\lambda}$ edge to obtain the following picture

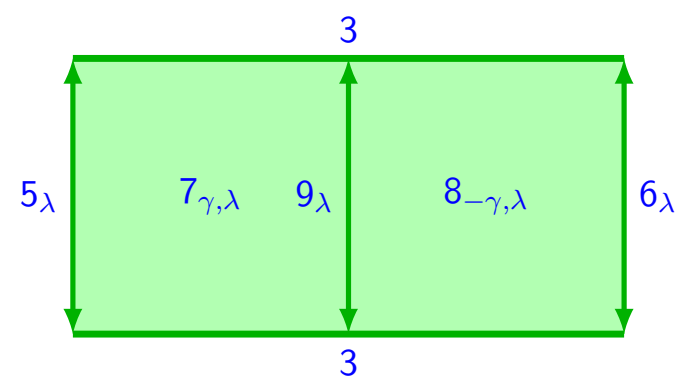

We now glue the top and bottom edges to arrive at the following cylinder:

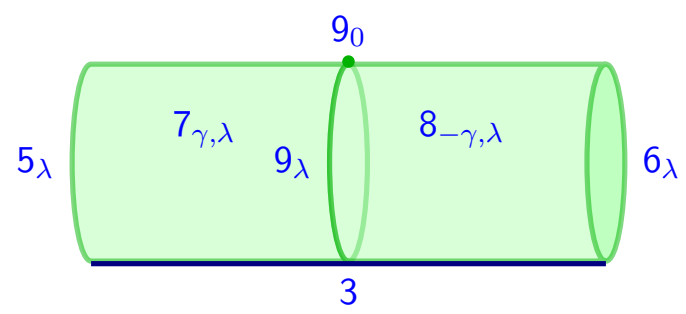

Finally, we collapse the "edge" labelled 3 to a point, arriving at the object in figure 1.

\subsection{Aristotelian limits}

There are two kinds of superisations of aristotelian spacetimes: the ones where $\boldsymbol{B}$ acts as R-symmetries and the ones where $\boldsymbol{B}$ acts trivially. We treat them in turn.

\subsubsection{Aristotelian superspaces with R-symmetry}

The homogeneous spacetimes $\mathbb{R} \times H^{3}$ and $\mathbb{R} \times S^{3}$ underlying the homogeneous superspaces SM14-SM17 have S as their limit. Therefore, we could expect SM14-SM17 to have either SM18 or SM19 as limits. The relevant contraction uses the transformation

$$
g_{t} \cdot \boldsymbol{B}=\boldsymbol{B}, \quad g_{t} \cdot H=H \quad \text { and } \quad g_{t} \cdot \boldsymbol{P}=t \boldsymbol{P} .
$$

Taking the limit $t \rightarrow 0$, the $[\boldsymbol{P}, \boldsymbol{P}]$ bracket vanishes leaving all other brackets unchanged. Thus, we find SM14 $\rightarrow$ SM18, SM16 $\rightarrow$ SM18, SM15 $\rightarrow$ SM19 and SM17 $\rightarrow$ SM19.

Taking into account the form of $\mathfrak{h}$, and the $[\boldsymbol{Q}, \boldsymbol{Q}]$ bracket for each of these superspaces, we notice that each homogeneous spacetime has two superspaces associated with it. One for which

$$
\mathbb{b}=\frac{1}{2} \quad \text { and } \quad[\mathrm{Q}(s), \mathrm{Q}(s)]=|s|^{2} H,
$$

and one for which

$$
\mathbb{b}=\frac{1}{2}, \quad \mathbb{h}=\frac{1}{2} \mathbb{k} \quad \text { and } \quad[\mathrm{Q}(s), \mathrm{Q}(s)]=|s|^{2} H-\mathrm{B}(s \mathbb{k} \bar{s}) .
$$


Using transformations which act as

$$
g_{t} \cdot H=t H, \quad g_{t} \cdot \boldsymbol{Q}=\sqrt{t} \boldsymbol{Q}
$$

and trivially on $\boldsymbol{J}, \boldsymbol{B}$, and $\boldsymbol{P}$, we find the brackets of the latter superspaces described by

$$
\mathbb{b}=\frac{1}{2}, \quad \mathbb{h}=\frac{t}{2} \mathbb{k}, \quad \text { and } \quad[\mathrm{Q}(s), \mathrm{Q}(s)]=|s|^{2} H-t \mathrm{~B}(s \mathbb{k} \bar{s}) .
$$

Therefore, taking the limit $t \rightarrow 0$, we find the former superspaces. Thus, we get the limits $\mathrm{SM} 15 \rightarrow \mathrm{SM} 14, \mathrm{SM} 17 \rightarrow \mathrm{SM} 16$ and $\mathrm{SM} 19 \rightarrow \mathrm{SM} 18$.

All of the above superspaces have SM18 as a limit. Therefore, we will only consider the limits of this superspace to those aristotelian superspaces without R-symmetry. Letting

$$
g_{t} \cdot \boldsymbol{B}=t \boldsymbol{B}, \quad g_{t} \cdot H=H, \quad g_{t} \cdot \boldsymbol{P}=\boldsymbol{P}, \quad g_{t} \cdot \boldsymbol{Q}=\boldsymbol{Q},
$$

and taking the limit $t \rightarrow 0$, we arrive at a non-effective super Lie pair corresponding to SM22.

\subsubsection{Aristotelian superspaces without R-symmetry}

The aristotelian homogeneous spacetimes $\mathbb{R} \times S^{3}, \mathbb{R} \times H^{3}$, and TS have $\mathbf{S}$ as their limit; therefore, we would expect their superisations to have have one or more of SM20-SM23 as limits. For TS to have $S$ as its limit, we require the transformation

$$
g_{t} \cdot \boldsymbol{B}=\boldsymbol{B}, \quad g_{t} \cdot H=t H \quad \text { and } \quad g_{t} \cdot \boldsymbol{P}=\boldsymbol{P} .
$$

Wanting to ensure $[\boldsymbol{Q}, \boldsymbol{Q}] \neq 0$, and that the limit $t \rightarrow 0$ is well-defined, we need $g_{t} \cdot \boldsymbol{Q}=$ $\sqrt{t} \boldsymbol{Q}$. Taking this limit, we find $\mathrm{SM} 24_{\lambda} \rightarrow \mathrm{SM} 21$.

To get $\mathrm{S}$ from $\mathbb{R} \times S^{3}$, we need the transformation

$$
g_{t} \cdot \boldsymbol{B}=\boldsymbol{B}, \quad g_{t} \cdot H=H \quad \text { and } \quad g_{t} \cdot \boldsymbol{P}=t \boldsymbol{P} .
$$

Using this transformation and taking the limit $t \rightarrow 0$, we find SM25 $\rightarrow$ SM22. However, the limit is not well-defined for SM26 due to $\boldsymbol{P}$ in the expression for $[\boldsymbol{Q}, \boldsymbol{Q}]$. In this case, we additionally require $g_{t} \cdot \boldsymbol{Q}=\sqrt{t} \boldsymbol{Q}$. Then SM26 $\rightarrow$ SM20. Another choice of transformation,

$$
g_{t} \cdot \boldsymbol{B}=\boldsymbol{B}, \quad g_{t} \cdot H=t H, \quad g_{t} \cdot \boldsymbol{P}=t \boldsymbol{P} \quad \text { and } \quad g_{t} \boldsymbol{Q}=\sqrt{t} \boldsymbol{Q},
$$

for SM26, gives SM23 in the limit $t \rightarrow 0$. Thus, we also have SM26 $\rightarrow$ SM23.

Finally, to get $\mathrm{S}$ from $\mathbb{R} \times H^{3}$, we use the transformation

$$
g_{t} \cdot \boldsymbol{B}=\boldsymbol{B}, \quad g_{t} \cdot H=H, \quad g_{t} \cdot \boldsymbol{P}=t \boldsymbol{P} .
$$

To ensure the limit $t \rightarrow 0$ is well-defined, we subsequently need $g_{t} \cdot \boldsymbol{Q}=\sqrt{t} \boldsymbol{Q}$. This transformation with the limit gives SM27 $\rightarrow$ SM21.

There are only two underlying aristotelian homogeneous spacetimes which have more than one superisation. These are $\mathrm{S}$ and $\mathbb{R} \times S^{3}$. In the latter case, we find the superisation SM25 as the limit of SM26 using the transformation

$$
g_{t} \cdot \boldsymbol{B}=\boldsymbol{B}, \quad g_{t} \cdot H=t H, \quad g_{t} \cdot \boldsymbol{P}=\boldsymbol{P} \quad \text { and } \quad g_{t} \cdot \boldsymbol{Q}=\sqrt{t} \boldsymbol{Q},
$$


and taking $t \rightarrow 0$. In the former case, the superisations SM22 and SM21 can be found as limits of SM23 using the transformations

$$
g_{t} \cdot \boldsymbol{B}=\boldsymbol{B}, \quad g_{t} \cdot H=t H, \quad g_{t} \cdot \boldsymbol{P}=\boldsymbol{P} \quad \text { and } \quad g_{t} \cdot \boldsymbol{Q}=\sqrt{t} \boldsymbol{Q},
$$

and

$$
g_{t} \cdot \boldsymbol{B}=\boldsymbol{B}, \quad g_{t} \cdot H=H, \quad g_{t} \cdot \boldsymbol{P}=t \boldsymbol{P} \quad \text { and } \quad g_{t} \cdot \boldsymbol{Q}=\sqrt{t} \boldsymbol{Q},
$$

respectively. We also have

$$
g_{t} \cdot \boldsymbol{B}=\boldsymbol{B}, \quad g_{t} \cdot H=t H, \quad g_{t} \cdot \boldsymbol{P}=\boldsymbol{P} \quad \text { and } \quad g_{t} \cdot \boldsymbol{Q}=\boldsymbol{Q},
$$

giving the limit SM20 $\rightarrow$ SM21.

\subsubsection{A non-contracting limit}

Use the following change of basis on the Lie superalgebra for $\mathrm{SM} 24_{\lambda}$,

$$
\boldsymbol{B}^{\prime}=\boldsymbol{B}, \quad H^{\prime}=2 \lambda^{-1} H, \quad \boldsymbol{P}^{\prime}=\boldsymbol{P}, \quad \boldsymbol{Q}^{\prime}=\boldsymbol{Q} .
$$

The brackets then become

$$
\left[H^{\prime}, \mathrm{P}(\pi)^{\prime}\right]=2 \lambda^{-1} \mathrm{P}(\pi)^{\prime}, \quad\left[H^{\prime}, \mathrm{Q}^{\prime}(s)\right]=\mathrm{Q}^{\prime}\left(s\left(\lambda^{-1}+\mathbb{k}\right)\right), \quad\left[\mathrm{Q}^{\prime}(s), \mathrm{Q}^{\prime}(s)\right]=-\mathrm{P}^{\prime}(s \mathbb{k} \bar{s}) .
$$

Taking the limits $\lambda \rightarrow \pm \infty$, we find the superspace SM20. Therefore, the line of superspaces $\mathrm{SM} 24_{\lambda}$ compactifies to a circle with SM20 as the point at infinity.

\subsection{Summary}

The picture resulting from the above discussion is given in figure 1. Except for SM3 $\rightarrow$ SM4, the limits from the families $\mathrm{SM} 5_{\lambda}, \mathrm{SM} 6_{\lambda}, \mathrm{SM} 7_{\gamma, \lambda}, \mathrm{SM} 8_{\gamma, \lambda}, \mathrm{SM} 9_{\lambda}$ and $\mathrm{SM} 11_{\chi}$ to $\mathrm{SM} 4$ are not shown explicitly in order to improve readability. Neither is the limit between $S M 24_{\lambda}$ and SM21 shown.

For comparison, we extract from ([4], figure 3) the subgraph corresponding to spacetimes which admit superisations and show it in figure 2. There are arrows between these two pictures: taking a superspace to its corresponding spacetime, but making this explicit seems beyond our combined artistic abilities.

Nevertheless, interpreting figures 1 and 2 as posets, with arrows defining the partial order, the map taking a superspace to its underlying spacetime is surjective by construction (we consider only superisable spacetimes) and order preserving, as shown at the start of this section. As can be gleaned from table 14, the fibres of this map are often quite involved, clearly showing the additional "internal" structure in the superspace which allows for more than one possible superisation of a spacetime.

We should mention that despite appearances, superspaces SM3 and SM4 share the same underlying spacetime: namely, the galilean spacetime G. Notice that superspaces SM21 and SM22, which are "terminal" in the partial order, correspond to the static aristotelian spacetime S. With the exception of $\lim _{\chi \rightarrow \infty} \mathrm{SM} 11_{\chi}=\mathrm{SM} 9_{0}$, all other non-contracting 

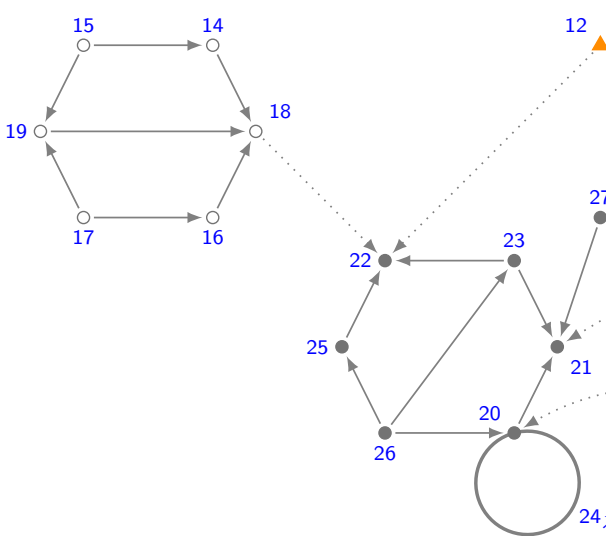

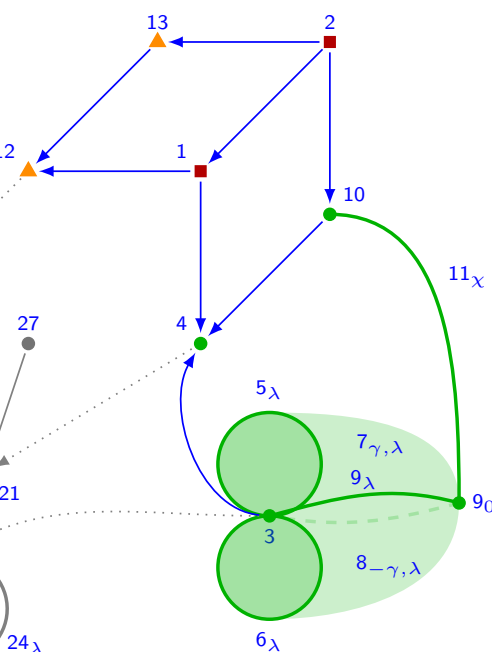

- lorentzian

- galilean

$\triangle$ carrollian

- aristotelian

$\circ$ aristotelian $+\mathrm{R}$

Figure 1. Homogeneous superspaces and their limits. (Numbers are hyperlinked to the corresponding superspaces in table 14.)

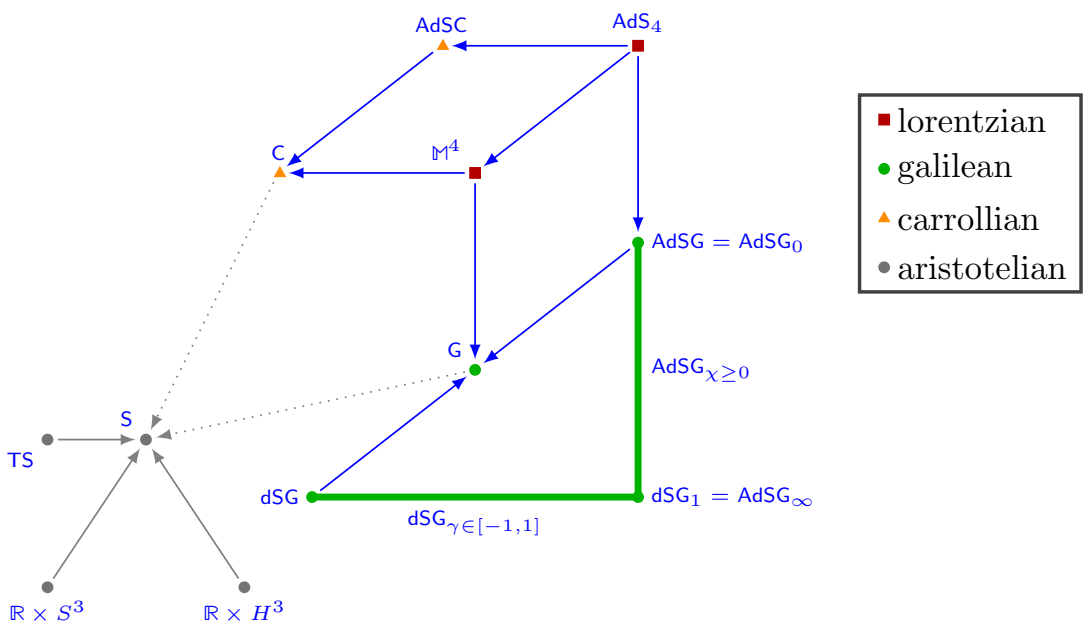

Figure 2. Limits between superisable spacetimes.

limits between superspaces induce limits between the underlying spacetimes which arise from contractions of the kinematical Lie algebras: the limits $|\lambda| \rightarrow \infty$ of $\mathrm{SM}_{\lambda}$ and $\mathrm{SM}_{\lambda}$ induce the contraction dSG $\rightarrow \mathrm{G}$, whereas the limits $|\lambda| \rightarrow \infty$ of $\mathrm{SM} 7_{\gamma, \lambda}, \mathrm{SM} 8_{\gamma, \lambda}$ and $\mathrm{SM} 9_{\lambda}$ induce the contractions $\mathrm{dSG}_{\gamma} \rightarrow \mathrm{G}$, where $\gamma=1$ for $\mathrm{SM} 9_{\lambda}$.

\section{Conclusions}

In this paper, we have answered the question: What are the possible super-kinematics? by classifying $(N=1 d=4)$ kinematical Lie superalgebras and their corresponding superspaces.

The Lie superalgebras were classified by solving the Jacobi identities in a quaternionic reformulation, which made the computations no harder than multiplying quaternions and 
paying close attention to the action of automorphisms in order to ensure that there is no repetition in our list. Since we are interested in supersymmetry, we focussed on Lie superalgebras where the supercharges were not abelian: i.e., we demand that $[\boldsymbol{Q}, \boldsymbol{Q}] \neq 0$ and, subject to that condition, we classified Lie superalgebras which extend either kinematical or aristotelian Lie algebras. The results are contained in tables 4 and 6, respectively.

There are two salient features of these classifications. Firstly, not every kinematical Lie algebra admits a supersymmetric extension: in some cases because of our requirement that $[\boldsymbol{Q}, \boldsymbol{Q}] \neq 0$, but in other cases (e.g., $\mathfrak{s o}(5), \mathfrak{s o}(4,1), \ldots$ ) because the four-dimensional spinor representation of $\mathfrak{s o}(3)$ does not extend to a representation of these Lie algebras.

Secondly, some kinematical Lie algebras admit more than one non-isomorphic supersymmetric extension. For example, the galilean Lie algebra admits two supersymmetric extensions, but only one of them (S8) can be obtained as a contraction of osp(1|4). By far most of the Lie superalgebras in our classification cannot be so obtained and hence are not listed in previous classifications. Nevertheless, our "moduli space" of Lie superalgebras is connected, if not always by contractions. For example, the other supersymmetric extension of the galilean algebra (S7) can be obtained as a non-contracting limit of some of the multi-parametric families of Lie superalgebras in the limit as one of the parameters goes to $\pm \infty$, in effect compactifying one of the directions in the parameter space into a circle.

We classified the corresponding superspaces via their super Lie pairs $(\mathfrak{s}, \mathfrak{h})$, where $\mathfrak{s}$ is a kinematical Lie superalgebra and $\mathfrak{h}$ an admissible subalgebra. Every such pair "superises" a pair $(\mathfrak{k}, \mathfrak{h})$, where $\mathfrak{k}=\mathfrak{s}_{\overline{0}}$ is a kinematical Lie algebra. As shown in [4], effective and geometrically realisable pairs $(\mathfrak{k}, \mathfrak{h})$ are in bijective correspondence with simply-connected homogeneous spacetimes, and hence the super Lie pairs $(\mathfrak{s}, \mathfrak{h})$ are in bijective correspondence with superisations of such spacetimes. These are listed in table 14.

There are several salient features of that table. Firstly, many spacetimes admit more than one inequivalent superisation. Whereas Minkowski and AdS spacetimes admit a unique $(N=1)$ superisation, and so too do the (superisable) carrollian spacetimes, many of the galilean spacetimes admit more than one and in some cases even a circle of superisations.

Secondly, there are effective super Lie pairs $(\mathfrak{s}, \mathfrak{h})$ for which the underlying pair $(\mathfrak{k}, \mathfrak{h})$ is not effective. This means that the "boosts" act trivially on the underlying spacetime, but nontrivially in the superspace: in other words, the "boosts" are actually R-symmetries. Since $(\mathfrak{k}, \mathfrak{h})$ is not effective, this means that it describes an aristotelian spacetime and this gives rise to the class of aristotelian superspaces with R-symmetry.

Thirdly, there are three superspaces in our list which also appear in [3]: namely, Minkowski (SM1) and AdS (SM2) superspaces, but also the aristotelian superspace SM26, whose underlying manifold appears in [3] as the lorentzian Lie group $\mathbb{R} \times \mathrm{SU}(2)$ with a bi-invariant metric.

Lastly, just like Minkowski $\left(M^{4}\right)$ and carrollian AdS (AdSC) spacetimes are homogeneous under the Poincaré group, their (unique) superisations (SM1 and SM13, respectively) are homogeneous under the Poincaré supergroup. This suggests a sort of correspondence or duality, which we hope to explore in future work.

There are a number of natural extensions to the results in this paper, which we list in no particular order. It would be interesting to classify extended $N>1$ superalgebras 
and superspaces in four dimensions and also kinematical/aristotelian superalgebras and superspaces in other dimensions: particularly in three-dimensions due to their use in ChernSimons theories (see, e.g., [22]). In the three-dimensional case, it would be important to determine the possible central charges and also the existence of invariant inner products. It would also be interesting to classify superconformal algebras along the lines of [23], which at least in four dimensions would be amenable to the quaternionic formalism employed in this paper. There has been a great deal of work on Schrödinger superalgebras, departing from the pioneering work in [24].

As shown in tables 4 and 6, many of these Lie superalgebras are graded and hence can serve as the starting ingredient to explore its filtered deformations, as advocated in [3, 25]; perhaps allowing us to go from the homogeneous models classified in this paper to more general superspaces.

The underlying spacetimes of the superspaces in table 14 are reductive and hence possess a canonical invariant connection. It is a natural question to ask whether the kinematical superalgebras admit an interpretation as Killing superalgebras in the spacetimes; that is, whether they are generated by "spinor" fields relative to some connection modifying the canonical invariant connection. In fact, as proved in ([18], section 5) in the context of spin manifolds, this is indeed the case (see Definition 5.3 in [18] for the notion of a generalised Killing spinor).

Finally, along the lines of ([18], section 4), we could investigate the invariant connections in the superspaces in table 14, by determining the space of Nomizu maps, as was done in [5] for the homogeneous spacetimes.

\section{Acknowledgments}

We are very grateful to Andrea Santi for answering many of our questions about homogeneous supermanifolds and Stefan Prohazka for comments on a previous version of this paper and which we hope to have incorporated in a way that has hopefully improved the exposition. We are also grateful to an anonymous referee for pointing out that we had missed some explanation concerning table 1. JMF was visiting the University of Stavanger during the final stages of writing this paper and he's grateful to Paul de Medeiros for lending his ear. He's also grateful to Paul and Sigbjørn Hervik for the invitation to visit and the hospitality.

\section{A Lorentzian superspaces}

In this appendix we give the definitions of the lorentzian superspaces, in a way that is as agnostic as possible about conventions. These are precisely the superspaces which also appear in [3], since their supersymmetry algebras are filtered deformations of subalgebras of the Poincaré superalgebra.

\section{A.1 Minkowski superspace}

The ur-example is, of course, Minkowski superspace (SM1), which is a homogeneous space of the Poincaré supergroup and can be described by a pair $(\mathfrak{s}, \mathfrak{h})$ as follows. The kine- 
matical Lie superalgebra $\mathfrak{s}$ is the $N=1$ Poincaré superalgebra, which is defined as follows. Let $(V, \eta)$ be a lorentzian ("mostly minus") four-dimensional vector space and let $\mathfrak{s o}(V)$ denote the skew-symmetric endomorphisms of $V$; that is, linear maps $\varphi: V \rightarrow V$ such that $\eta(\varphi(v), w)=-\eta(v, \varphi(w))$ for all $v, w \in V$. Let $C \ell(V)$ denote the corresponding Clifford algebra, with Clifford relation $v \cdot v=-\eta(v, v) \mathbb{1}$, for all $v \in V$. As a real associative algebra, $C \ell(V) \cong \operatorname{End}(S)$, where $S$ is a real four-dimensional irreducible Clifford module. It is also an irreducible representation ("Majorana spinors") of $\mathfrak{s o}(V) \subset C \ell(V)$; although its complexification ("Dirac spinors") decomposes into positive- and negative-chirality irreducible representations ("Weyl spinors"). On $S$ there is a symplectic inner product $\langle-,-\rangle$ satisfying

$$
\left\langle v \cdot s_{1}, s_{2}\right\rangle=-\left\langle s_{1}, v \cdot s_{2}\right\rangle,
$$

for all $s_{1}, s_{2} \in S$ and $v \in V$, where denotes the Clifford action. This implies that $\langle-,-\rangle$ is $\mathfrak{s o}(V)$-invariant. We define a $\mathbb{Z}$-graded vector space $\mathfrak{s}=\mathfrak{s}_{0} \oplus \mathfrak{s}_{-1} \oplus \mathfrak{s}_{-2}$, with $\mathfrak{s}_{0}=\mathfrak{s o}(V)$, $\mathfrak{s}_{-1}=S$ and $\mathfrak{s}_{-2}=V$. Let $\mathfrak{s}_{\overline{0}}=\mathfrak{s}_{0} \oplus \mathfrak{s}_{-2}$ and $\mathfrak{s}_{\overline{1}}=\mathfrak{s}_{1}$ and we define on the vector superspace $\mathfrak{s}=\mathfrak{s}_{\overline{0}} \oplus \mathfrak{s}_{\overline{1}}$ the structure of a Lie superalgebra as follows. The Lie algebra structure on $\mathfrak{s}_{\overline{0}}$ is the Poincaré algebra:

$$
[(A, v),(B, w)]=(A B-B A, A(w)-B(v)),
$$

or equivalently,

$$
[A, B]=A B-B A, \quad[A, v]=A(v)=-[v, A] \quad \text { and } \quad[v, w]=0,
$$

for $A, B \in \mathfrak{s o}(V)$ and $v, w \in V$. We make $\mathfrak{s}_{\overline{1}}$ into an $\mathfrak{s}_{\overline{0}}$-module by declaring $\mathfrak{s o}(V)$ to act via the spinor representation and $V$ to act trivially. Finally, if $s_{1}, s_{2} \in \mathfrak{s}_{\overline{1}}$, their bracket $\left[s_{1}, s_{2}\right] \in V$ is defined to be the vector such that, for all $v \in V$,

$$
\eta\left(\left[s_{1}, s_{2}\right], v\right)=\left\langle s_{1}, v \cdot s_{2}\right\rangle,
$$

which is symmetric by equation (A.1) and the fact that $\langle-,-\rangle$ is symplectic. The bracket defines a symmetric bilinear map $\mathfrak{s}_{\overline{1}} \times \mathfrak{s}_{\overline{1}} \rightarrow \mathfrak{s}_{\overline{0}}$ or, equivalently, a linear map $\bigodot^{2} \mathfrak{s}_{\overline{1}} \rightarrow V \subset \mathfrak{s}_{\overline{0}}$ from the symmetric tensor square of $\mathfrak{s}_{\overline{1}}$. This map is surjective and, moreover, $\mathfrak{s o}(V)$ equivariant because $\eta,\langle-,-\rangle$ are $\mathfrak{s o}(V)$-invariant and Clifford action is $\mathfrak{s o}(V)$-equivariant. The Jacobi identity $[[s, s], s]=0$ is trivially satisfied because $[s, s] \in V$ and $V$ acts trivially on $S$. This defines the Poincaré superalgebra $\mathfrak{s}$. The admissible subalgebra $\mathfrak{h}=\mathfrak{s o}(V)$ is the Lie subalgebra of Lorentz transformations, and Minkowski superspace is described by the pair $(\mathfrak{s}, \mathfrak{h})$. The pair $\left(\mathfrak{s}_{0}, \mathfrak{h}\right)$ defines a homogeneous spacetime, which is none other than Minkowski spacetime $\mathbb{M}^{4}$.

\section{A.2 Anti de Sitter superspace}

The second well-known example is anti de Sitter superspace (SM2), whose associated kinematical Lie superalgebra is isomorphic to $\mathfrak{o s p}(1 \mid 4)$ and whose construction we now review. The spin representation of $\mathfrak{s o}(3,2)$ defines an isomorphism $\mathfrak{s o}(3,2) \rightarrow \mathfrak{s p}(4, \mathbb{R})$. This means that the spinor representation $S$ is real, symplectic and four-dimensional. Let $\langle-,-\rangle$ denote the $\mathfrak{s o}(3,2)$-invariant symplectic inner product on $S:\left\langle X \cdot s_{1}, s_{2}\right\rangle=-\left\langle s_{1}, X \cdot s_{2}\right\rangle$ for 
all $s_{1}, s_{2} \in S$ and $X \in \mathfrak{s o}(3,2)$. Let $\kappa$ denote the Killing form on $\mathfrak{s o}(3,2)$, which is nondegenerate because $\mathfrak{s o}(3,2)$ is simple. Define a bilinear map $[-,-]: S \times S \rightarrow \mathfrak{s o}(3,2)$ by declaring $\left[s_{1}, s_{2}\right] \in \mathfrak{s o}(3,2)$ to be the unique element whose inner product (relative to the Killing form) with any $X \in \mathfrak{s o}(3,2)$ is given by

$$
\kappa\left(\left[s_{1}, s_{2}\right], X\right)=\left\langle s_{1}, X \cdot s_{2}\right\rangle,
$$

which is symmetric by the $\mathfrak{s o}(3,2)$-invariance of the symplectic structure. Define a vector superspace $\mathfrak{s}=s_{\overline{0}} \oplus \mathfrak{s}_{\overline{1}}$, with $\mathfrak{s}_{\overline{0}}=\mathfrak{s o}(3,2)$ and $\mathfrak{s}_{\overline{1}}=S$ and an even bracket on $\mathfrak{s}$ by taking it to be the Lie bracket on $\mathfrak{s}_{0}$, the action of $\mathfrak{s o}(3,2)$ on $S$ and the above map $\bigodot^{2} S \rightarrow \mathfrak{s o}(3,2)$. The Jacobi identity follows from the fact that $\mathfrak{s}_{\overline{0}}$ is a Lie algebra, $\mathfrak{s}_{\overline{1}}$ is an $\mathfrak{s}_{\overline{0}}$-module, the bracket $\bigodot^{2} \mathfrak{s}_{\overline{1}} \rightarrow \mathfrak{s}_{\overline{0}}$ is $\mathfrak{s}_{\overline{0}}$-equivariant (since $\kappa$ and $\langle-,-\rangle$ are $\mathfrak{s}_{\overline{0}}$-invariant) and because the only $\mathfrak{s o}(3,2)$-equivariant linear map $\bigodot^{3} S \rightarrow S$ is the zero map. Notice that $[S, S]$ is a nonzero ideal of $\mathfrak{s o}(3,2)$, but since $\mathfrak{s o}(3,2)$ is simple, this is all of $\mathfrak{s o}(3,2)$. The resulting simple Lie superalgebra is isomorphic to $\mathfrak{o s p}(1 \mid 4)$. We may take for the admissible subalgebra $\mathfrak{h}$ the stabiliser in $\mathfrak{s o}(3,2)$ of any timelike vector in $\mathbb{R}^{3,2}$, which is isomorphic to $\mathfrak{s o}(3,1) \subset \mathfrak{s o}(3,2)$. The pair $(\mathfrak{s}, \mathfrak{h})=(\mathfrak{o s p}(1 \mid 4), \mathfrak{s o}(3,1))$ defines a homogeneous superspace whose underlying homogeneous spacetime $\left(\mathfrak{s}_{\overline{0}}, \mathfrak{h}\right)=(\mathfrak{s o}(3,2), \mathfrak{s o}(3,1))$ is of course anti de Sitter spacetime $\mathrm{AdS}_{4}$.

\section{A.3 Einstein static superspace}

The third and final example of a lorentzian superspace in our classification is the aristotelian superspace SM26, which is one of the superisations of the Einstein static universe $\mathbb{R} \times S^{3}$. We shall be brief and refer to ([3], Thm. 14) for the details, particularly equation (98) in that paper, except that what we call $\mathfrak{h}$ in that paper is not the admissible subalgebra as in this paper, but actually the rotational subalgebra $r$. The notation is as in the case of the Minkowski superspace treated above: $(V, \eta)$ a "mostly minus" lorentzian four-dimensional vector space and $S$ the real four-dimensional irreducible $C \ell(V)$-module. Pick a nonzero timelike vector $\varphi \in V$, whose stabiliser in $\mathfrak{s o}(V)$ is the rotational subalgebra $\mathfrak{r}$. Define $\widetilde{\psi}: V \rightarrow \mathfrak{r}$ by $\widetilde{\psi}(v)=2 \imath_{v} \imath_{\varphi}$ vol $\in \wedge^{2} V \cong \mathfrak{s o}(V)$. Since for $w \in V, \widetilde{\psi}(v) w=2 \imath_{w} \imath_{v} \imath_{\varphi}$ vol, we see that $\widetilde{\psi}(v) \varphi=0$ and hence $\widetilde{\psi}(v) \in \mathfrak{r}$ for all $v \in V$ as claimed. Now let $A, B \in \mathfrak{r}$, $v, w \in V$ and $s \in S$. The Lie brackets $[A, B],[A, s],[A, v]$ and $[s, s]$ are exactly as in the Poincaré superalgebra, whereas

$$
[v, w]=\widetilde{\psi}(v) w-\widetilde{\psi}(w) v \quad \text { and } \quad[v, s]=-\frac{1}{2}(v \cdot \varphi+3 \varphi \cdot v) \cdot s+\widetilde{\psi}(v) s .
$$

Let us choose a pseudo-orthonormal basis $\left(\boldsymbol{e}_{0}, \boldsymbol{e}_{1}, \boldsymbol{e}_{2}, \boldsymbol{e}_{3}\right)$ for $V$ and take $\varphi=\boldsymbol{e}_{0}$. Then we have that $\widetilde{\psi}\left(\boldsymbol{e}_{0}\right)=0$ and so $\left[\boldsymbol{e}_{0}, \boldsymbol{e}_{i}\right]=0$ for all $i=1,2,3$. It follows that $\widetilde{\psi}\left(\boldsymbol{e}_{i}\right)=$ $-2 \epsilon_{i j k} \boldsymbol{e}_{j} \wedge \boldsymbol{e}_{k}$ and hence $\left[\boldsymbol{e}_{i}, \boldsymbol{e}_{j}\right]=4 \epsilon_{i j k} \boldsymbol{e}_{k}$. Acting on $s \in S,\left[\boldsymbol{e}_{0}, s\right]=2 \mathrm{vol} s$. Calculating from the above formula, $\left[\boldsymbol{e}_{i}, s\right]=-\epsilon_{i j k}\left(\boldsymbol{e}_{j} \wedge \boldsymbol{e}_{k}\right) \cdot s$. Letting $P_{i}=\frac{1}{2} \boldsymbol{e}_{i}-J_{i}$, we find that $\left[P_{i}, P_{j}\right]=\epsilon_{i j k} J_{k}$ and that $P_{i}$ and $J_{i}$ act in the same way on $S$. Choosing $\boldsymbol{e}_{0}=-2 H$ and $\boldsymbol{e}_{i}=2\left(J_{i}+P_{i}\right)$, we find that (rescaling $s$ ) the $[s, s]$ bracket is precisely the one in SM26.

Open Access. This article is distributed under the terms of the Creative Commons Attribution License (CC-BY 4.0), which permits any use, distribution and reproduction in any medium, provided the original author(s) and source are credited. 


\section{References}

[1] Yu. A. Golfand and E.P. Likhtman, Extension of the Algebra of Poincaré Group Generators and Violation of p Invariance, JETP Lett. 13 (1971) 323 [INSPIRE].

[2] B. Zumino, Nonlinear Realization of Supersymmetry in de Sitter Space, Nucl. Phys. B 127 (1977) 189 [INSPIRE].

[3] P. de Medeiros, J. Figueroa-O'Farrill and A. Santi, Killing superalgebras for Lorentzian four-manifolds, JHEP 06 (2016) 106 [arXiv:1605.00881] [INSPIRE].

[4] J. Figueroa-O'Farrill and S. Prohazka, Spatially isotropic homogeneous spacetimes, JHEP 01 (2019) 229 [arXiv:1809.01224] [INSPIRE].

[5] J. Figueroa-O'Farrill, R. Grassie and S. Prohazka, Geometry and BMS Lie algebras of spatially isotropic homogeneous spacetimes, JHEP 08 (2019) 119 [arXiv:1905.00034] [INSPIRE].

[6] H. Bacry and J. Levy-Leblond, Possible kinematics, J. Math. Phys. 9 (1968) 1605 [InSPIRE].

[7] H. Bacry and J. Nuyts, Classification of Ten-dimensional Kinematical Groups With Space Isotropy, J. Math. Phys. 27 (1986) 2455 [INSPIRE].

[8] J. Rembielinski and W. Tybor, Possible superkinematics, Acta Phys. Polon. B 15 (1984) 611 [INSPIRE].

[9] V. Hussin, J. Negro and M.A. del Olmo, Kinematical superalgebras, J. Phys. A 32 (1999) 5097.

[10] R. Campoamor-Stursberg and M. Rausch de Traubenberg, Kinematical superalgebras and Lie algebras of order 3, J. Math. Phys. 49 (2008) 063506 [arXiv:0801.2630] [InSPIRE].

[11] C.-G. Huang and L. Li, Possible Supersymmetric Kinematics, Chin. Phys. C 39 (2015) 093103 [arXiv: 1409.5498] [INSPIRE].

[12] R. Puzalowski, Galilean supersymmetry, Acta Phys. Austriaca 50 (1978) 45 [InSPIRE].

[13] F. Palumbo, Nonrelativistic Supersymmetry, in Proceedings of the International Conference on Recent Progress in Many Body Theories, International Center for Theoretical Physics, Trieste Italy (1978), pg. 582.

[14] T.E. Clark and S.T. Love, Nonrelativistic supersymmetry, Nucl. Phys. B 231 (1984) 91 [INSPIRE].

[15] J.A. de Azcarraga and D. Ginestar, Nonrelativistic limit of supersymmetric theories, J. Math. Phys. 32 (1991) 3500 [INSPIRE].

[16] J.M. Figueroa-O'Farrill, Kinematical Lie algebras via deformation theory, J. Math. Phys. 59 (2018) 061701 [arXiv:1711.06111] [INSPIRE].

[17] G. Hochschild and J.-P. Serre, Cohomology of Lie algebras, Ann. Math. 57 (1953) 591.

[18] A. Santi, Superization of Homogeneous Spin Manifolds and Geometry of Homogeneous Supermanifolds, Abh. Math. Sem. Univ. Hamburg 80 (2010) 87 [arXiv:0905.3832] [INSPIRE].

[19] B. Kostant, Graded manifolds, graded Lie theory, and prequantization, in Conference on Differential Geometrical Methods in Mathematical Physics, Bonn Germany (1975), Lecture Notes Math. 570 (1977) 177. 
[20] M. Batchelor, The structure of supermanifolds, Trans. Am. Math. Soc. 253 (1979) 329.

[21] J.-L. Koszul, Graded manifolds and graded Lie algebras, in Proceedings of the international meeting on geometry and physics, Florence Italy (1982), Pitagora, Bologna Italy (1983), pg. 71.

[22] J. Matulich, S. Prohazka and J. Salzer, Limits of three-dimensional gravity and metric kinematical Lie algebras in any dimension, JHEP 07 (2019) 118 [arXiv:1903.09165] [INSPIRE].

[23] J.M. Figueroa-O'Farrill, Conformal Lie algebras via deformation theory, J. Math. Phys. 60 (2019) 021702 [arXiv:1809.03603] [INSPIRE].

[24] C. Duval and P.A. Horvathy, On Schrödinger superalgebras, J. Math. Phys. 35 (1994) 2516 [hep-th/0508079] [INSPIRE].

[25] P. de Medeiros, J. Figueroa-O'Farrill and A. Santi, Killing superalgebras for Lorentzian six-manifolds, J. Geom. Phys. 132 (2018) 13 [arXiv:1804.00319] [INSPIRE]. 